Portland State University

PDXScholar

$1-2014$

\title{
Wider Dissemination of Household Travel Survey Data Using Geographical Perturbation Methods
}

Kelly J. Clifton

Portland State University

Steven R. Gehrke

Portland State University

Follow this and additional works at: https://pdxscholar.library.pdx.edu/cengin_fac

Part of the Civil Engineering Commons, Environmental Engineering Commons, and the Transportation Commons

Let us know how access to this document benefits you.

\section{Citation Details}

Clifton, Kelly J. and Gehrke, Steven R., "Wider Dissemination of Household Travel Survey Data Using Geographical Perturbation Methods". OTREC-RR-489. Portland, OR: Transportation Research and Education Center (TREC) 2014. http://dx.doi.org/10.15760/trec.116

This Report is brought to you for free and open access. It has been accepted for inclusion in Civil and Environmental Engineering Faculty Publications and Presentations by an authorized administrator of PDXScholar. Please contact us if we can make this document more accessible: pdxscholar@pdx.edu. 


\section{G) OTREC}

FINAL REPORT

\section{Wider Dissemination of Household Travel Survey Data Using Geographical Perturbation Methods}

OTREC-RR-489

January 2014 



\title{
WIDER DISSEMINATION OF HOUSEHOLD TRAVEL SURVEY DATA USING GEOGRAPHICAL PERTURBATION METHODS
}

\author{
Draft Report
}

\section{OTREC-RR-489}

by

Kelly J. Clifton, Ph.D.

Portland State University

Steven R. Gehrke

Portland State University

for

Oregon Transportation Research

and Education Consortium (OTREC)

P.O. Box 751

Portland, OR 97207

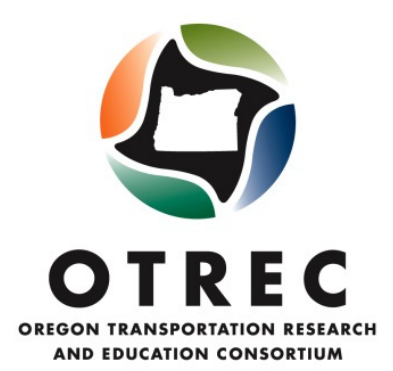

January 2014 



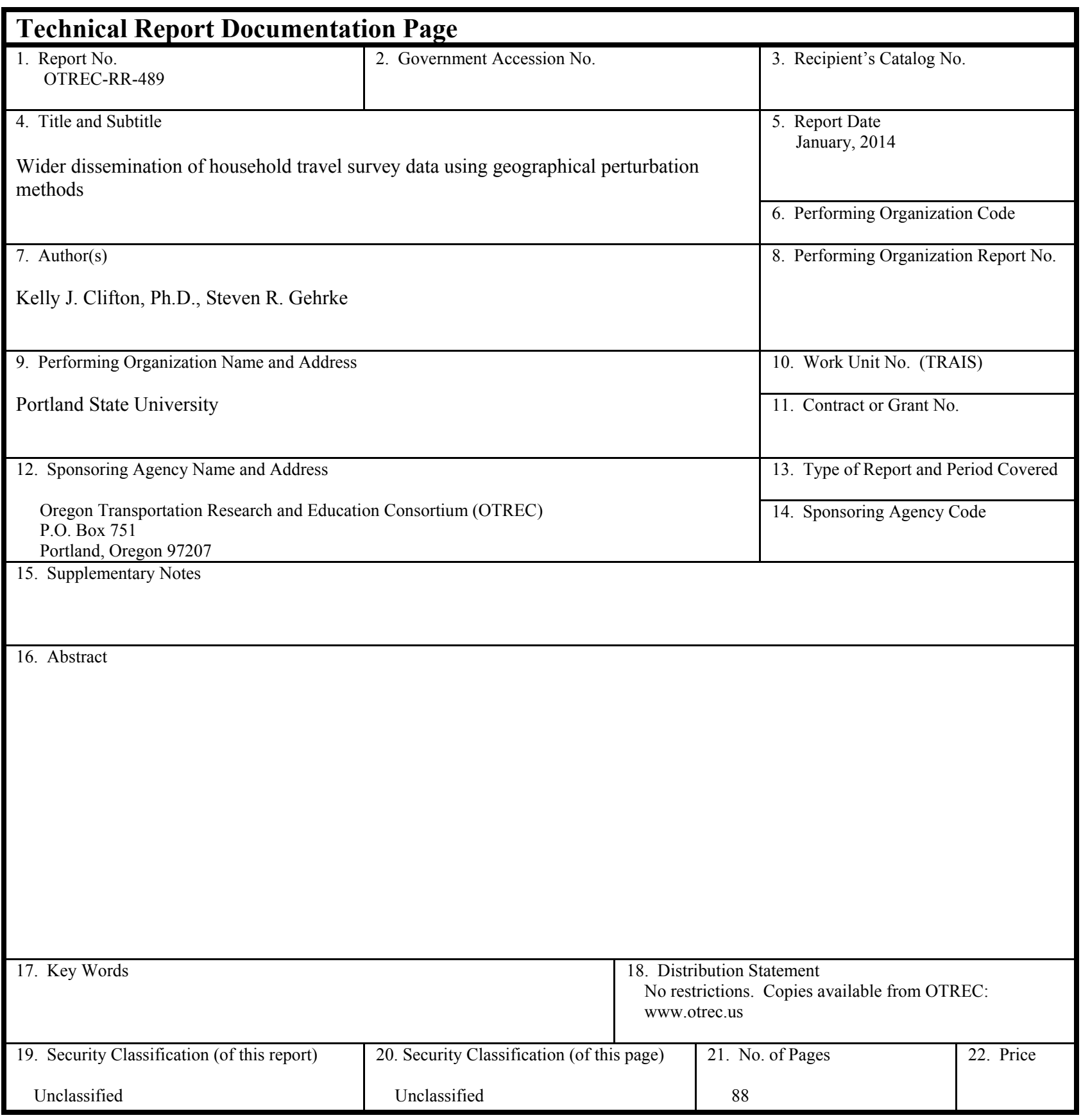




\section{ACKNOWLEDGEMENTS}

The authors wish to thank members of the Oregon Transportation Research and Education Consortium (OTREC) for their support of this project as well as the Oregon Modeling Steering Committee, Oregon Department of Transportation, and Portland State University's Institute of Portland Metropolitan Studies. Additionally, the authors are thankful for the assistance provided by Nebahat Noyan, Ph.D., and April Cutter as well as the vision of Keith Lawton, whose creative thought inspired the inception of this project.

\section{DISCLAIMER}

The contents of this report reflect the views of the authors, who are solely responsible for the facts and the accuracy of the material and information presented herein. This document is disseminated under the sponsorship of the U.S. Department of Transportation University Transportation Centers Program in the interest of information exchange. The U.S. Government assumes no liability for the contents or use thereof. The contents do not necessarily reflect the official views of the U.S. Government. This report does not constitute a standard, specification, or regulation. 


\section{TABLE OF CONTENTS}

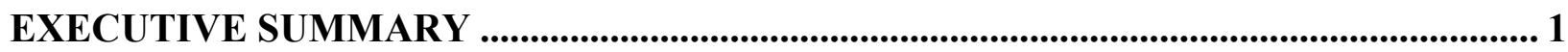

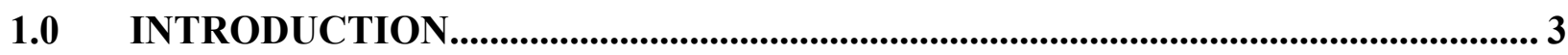

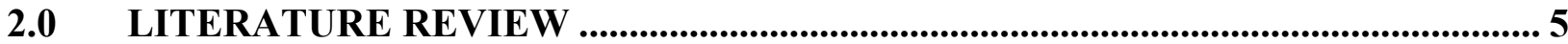

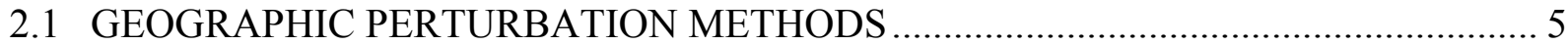

2.2 EVALUATION OF GEOGRAPHIC PERTURBATION METHODS ............................... 7

2.3 TRAVEL BEHAVIOR AND THE BUILT ENVIRONMENT......................................... 8

3.0 THEORETICAL FRAMEWORK FOR DISCLOSURE RISK AND DATA

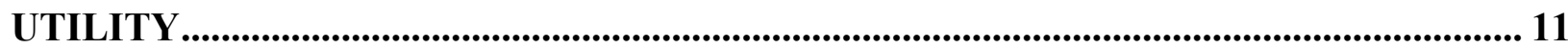

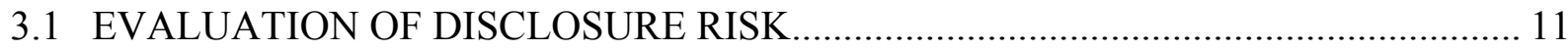

3.2 EVALUATION OF DATA UTILITY ........................................................................... 15

3.3 IMPLEMENTATION OF A GEOGRAPHIC PERTURBATION TECHNIQUE............ 16

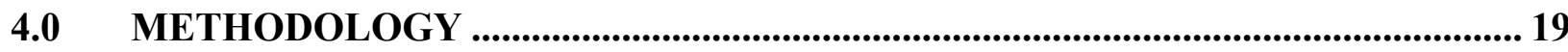

4.1 RANDOM GEOGRAPHIC PERTURBATION TECHNIQUE ..................................... 19

4.2 QUANTIFYING DISCLOSURE RISK AND DATA UTILITY ................................... 22

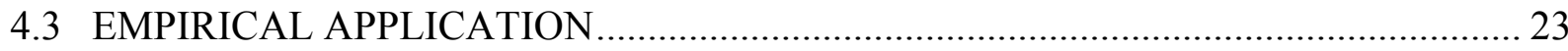

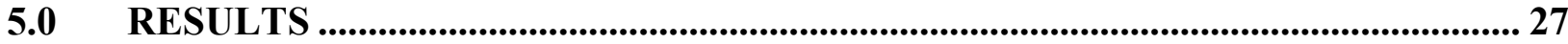

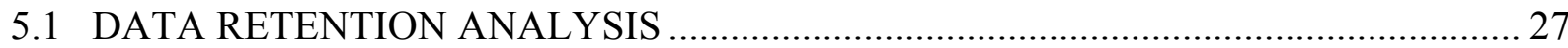

5.1.1 Data Retention within Subcategories of Population Density .................................. 28

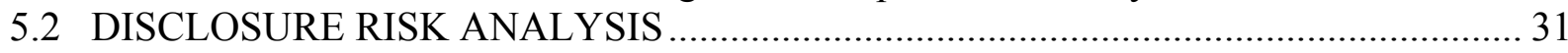

5.2.1 Disclosure Risk within Subcategories of Density Measures ………………………... 34

5.2.2 Disclosure Risk within Subcategories of Design Measures …………………......... 37

5.2.3 Disclosure Risk within Subcategories of Distance to Transit Measures .................. 39

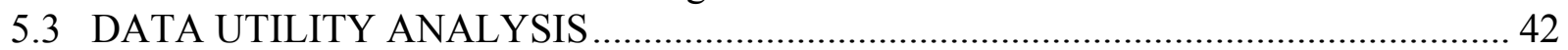

5.3.1 Data Utility within Subcategories of Density Measures............................................ 44

5.3.2 Data Utility within Subcategories of Design Measures ............................................... 47

5.3.3 Data Utility within Subcategories of Distance to Transit Measures.......................... 49

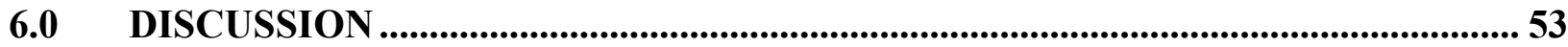

6.1 GENERAL RULES OF THUMB FOR FUTURE APPLICATION …………................5 53

6.2 LIMITATIONS AND POTENTIAL FUTURE EXPANSIONS...................................... 54

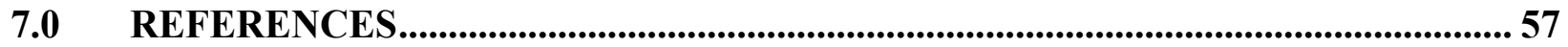

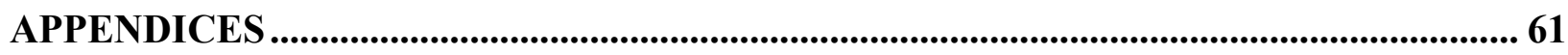

APPENDIX A: DONUT MASKING TECHNIQUE POSTGIS CODE ................................... 61 


\section{LIST OF TABLES}

Table 5-1: Total number of households retained within each scenario .................................... 27

Table 5-2: Percent of households retained within each scenario ............................................. 28

Table 5-3: Number and percent of retained households per scenario within class 1 for population

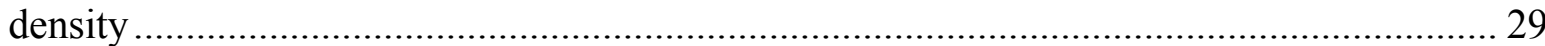

Table 5-4: Number and percent of retained households per scenario within class 2 for population density ..................................................................................................... 30

Table 5-5: Number and percent of retained households per scenario within class 3 for population

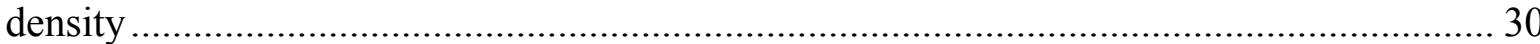

Table 5-6: Number and percent of retained households per scenario within class 4 for population

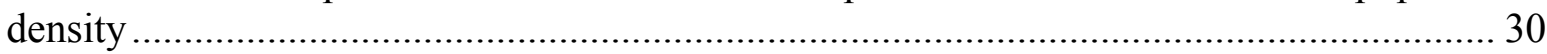

Table 5-7: Number and percent of retained households per scenario within class 5 for population

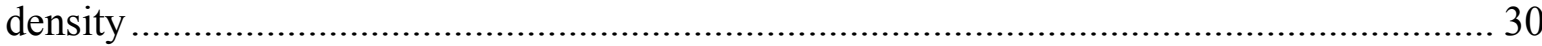

Table 5-8: Average minimum and maximum k-anonymity statistic from 50 simulations per scenario 32

Table 5-9: Average median and mean k-anonymity statistic from 50 simulations per scenario.. 32 


\section{LIST OF FIGURES}

Figure 3-1: Hierarchical framework of disclosure risk and data utility .................................. 12

Figure 4-1: Conceptual steps in applying the donut masking technique .................................... 20

Figure 4-2: Three-county study region of Portland metropolitan region...................................... 24

Figure 5-1: Quintile classification of population density for U.S. Census tracts in Portland

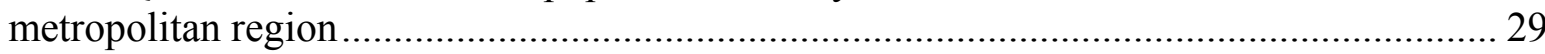

Figure 5-2: Level of anonymity per scenario over 50 simulations (average median k-anonymity)

Figure 5-3: Quintile classification of employment density for U.S. Census tracts in Portland

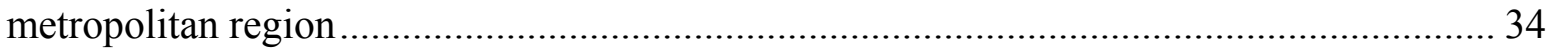

Figure 5-4: Level of anonymity per scenario over 50 simulations (quintiles for population

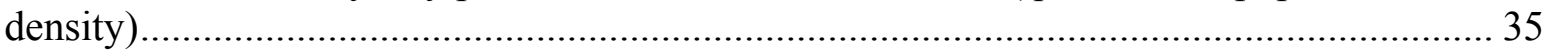

Figure 5-5: Level of anonymity per scenario over 50 simulations (quintiles for employment

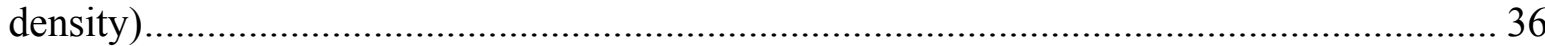

Figure 5-6: Natural break classification of intersection density for U.S. Census tracts in Portland

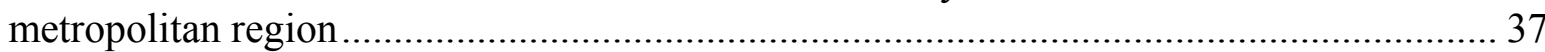

Figure 5-7: Level of anonymity per scenario over 50 simulations (natural breaks for intersection

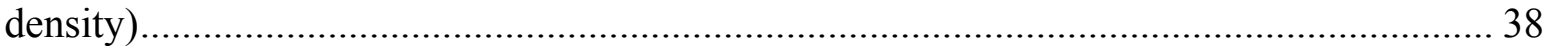

Figure 5-8: Manual classification of straight-line distance to nearest bus stop in Portland

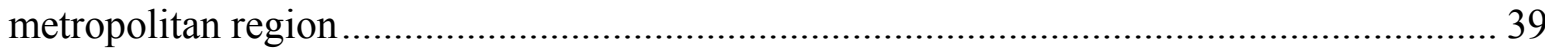

Figure 5-9: Manual classification of straight-line distance to nearest rail station in Portland

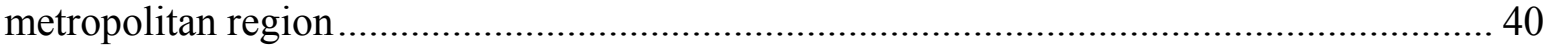

Figure 5-10: Level of anonymity per scenario over 50 simulations (manual breaks for bus stop distance).

Figure 5-11: Level of anonymity per scenario over 50 simulations (manual breaks for rail station

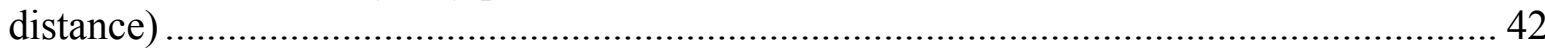

Figure 5-12: Level of spatial error per scenario over 50 simulations (all built environment

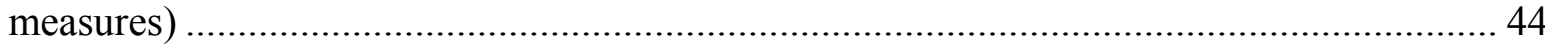

Figure 5-13: Level of spatial error per scenario over 50 simulations (quintiles for population

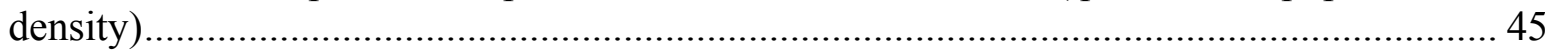

Figure 5-14: Level of spatial error per scenario over 50 simulations (quintiles for employment

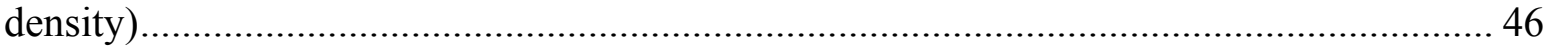

Figure 5-15: Level of spatial error per scenario over 50 simulations (natural breaks for intersection density) .................................................................................................. 48

Figure 5-16: Level of spatial error per scenario over 50 simulations (manual breaks for bus stop

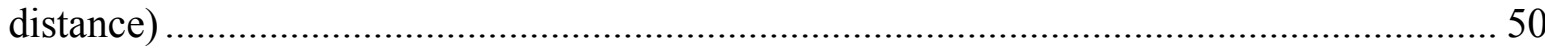

Figure 5-17: Level of spatial error per scenario over 50 simulations (manual breaks for rail

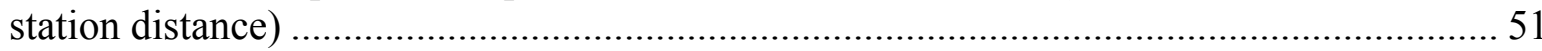




\section{EXECUTIVE SUMMARY}

Public agencies spend considerable resources collecting information about passenger travel in household travel surveys. These data are valuable for the rich and detailed information they provide, which contribute to regional and statewide travel demand models. These data have utility beyond travel demand modeling in their application to transportation policy and travel behavior research. As the demands on these data increase, so have the quantity of information collected. Detailed geospatial referencing of the home, work and other travel destinations are common practice and permit the integration with other spatially archived data sources, such as land use characteristics, transportation system information, and other built environment, social and economic data. Other public agencies, private consultancies, non-profits and educational institutions may benefit from access to the original data with applications to areas such as public health, equity, transportation safety and urban planning. But wide distribution of these important and expensive data is limited by the requirement to protect the confidentiality of survey participants, who are guaranteed anonymity in exchange for participation.

Given the constraint of anonymity, data are often aggregated to a geographic level such as census tracts or transportation analysis zones (TAZs) before being disseminated to the public, which limits the utility of this information. This is particularly true as the need for more spatially explicit information is needed for such areas as non-motorized planning, evaluation access to transit, local accessibility studies, health impact analysis and other interests in linking transportation outcomes to detailed spatial data.

To address these concerns, this project aims to examine an approach to permit dissemination of these spatially explicit data to a wider range of public constituents, while at the same time protecting the identities of study participants. To this end, this project will use geographical perturbation methods to add noise to the original data to protect confidentiality while at the same time allowing the detailed geo-spatial referencing to be included in the disseminated data. To do this, this research: (i) reviews geographical perturbation methods that seek to protect respondent confidentiality; (ii) outlines a framework for examining the disclosure risk in survey data; (iii) tests a procedure for implementing one promising perturbation practice, referred to as the donut masking technique, using data from a household activity travel survey in the Portland metropolitan region; and (iv) examines the disclosure risk and the error introduced to data derived from household location using this technique.

Results of this demonstration revealed that increases in the potential displacement distance of a geographically perturbed household generally reduced disclosure risk, but also limited data utility. Key guidelines from this study include:

- The ideal balance of minimum disclosure risk and maximum data utility should only be sought in the most urban contexts, as the configuration of such a donut for a less densely populated area will result in the significant loss of sampled observations. 
- For the greatest improvement in average levels of anonymity, the maximum displacement distance should be extended; however, the return on such an extension is less beneficial to a household in an urban context after one mile.

- An improvement in the utility of the dataset with regard to employment density of the respondent's census block group should be sought since this built environment measure was most impacted by the geographic perturbation of the actual household location.

- When seeking to maximize the utility of density measures of the built environment, the data custodian should be cognizant that the specification of a scenario with a maximum displacement distance over one mile may introduce excessive error in rural contexts.

- To provide an altered dataset giving adequate attention to both concepts of disclosure risk and data utility, the single specification of a donut masking technique to all respondents in the dataset should be avoided by a data custodian.

This study has shown the promise of the donut masking technique in negotiating the balance between disclosure risk and data utility that is central to the distribution of a household travel survey dataset with heightened spatial resolution. Nevertheless, the exploratory nature of this application of a new geographic perturbation technique has limitations and several questions are reserved for future expansion of this donut masking technique. 


\subsection{INTRODUCTION}

Collecting household travel survey data is an expensive process requiring the expenditure of vast resources from public agencies. The benefit of these efforts are far-reaching for practitioners and researchers as these collection processes provide detailed and rich data that inform regional and statewide travel demand applications. Over time, these public agencies have experienced added pressure to extend the utility of these data sources to policy and research applications exploring more complex interactions between the built environment and household travel behavior. Since these data, prior to any post-processing effort, provide disaggregate geographic representations of the survey respondent's home and work locations as well as an assortment of other spatial data identifying the household's travel patterns, they may then be augmented with other spatial data sources describing the surrounding environment to provide a more complete picture of what factors are associated with certain travel outcomes. Particularly, the capability of public agencies to offer these disaggregate household travel survey data improves the potential for researchers to improve their understanding of how non-motorized travel and the built environment are related. However, the release of data that may be spatially referenced to an individual household raises important concerns regarding the preservation of survey respondent confidentiality.

Historically, concerns regarding disclosure risk have been addressed in travel behavior research through the aggregation of these important and expensive data in order to adequately uphold the confidentiality pledge made between a survey respondent and administrator. Unfortunately, this practice of zonal aggregation compromises the utility of these datasets to researchers requiring a finer spatial representation to better understand the connection between non-motorized travel and the built environment since many of these trips often occur over shorter distances. Thus, there is a great benefit to providing these household travel data as a disaggregate representation. While largely unaddressed in travel behavior research, the negotiation of this barrier centered on minimizing the risk of identity disclosure for a survey respondent and ensuring the possibility of valid geographical analyses has received ample attention in other disciplines, which have sought to address this complication by the application of geographic perturbation methods. In general, a decrease in the potential for a breach of survey respondent confidentiality through the application of a geographic perturbation technique is directly associated with a decrease in the utility of the spatially altered dataset to the researcher. Although this relationship between disclosure risk and data utility has been established, there is no consensus on a single procedure for balancing these central concepts with the intention of aiding the dissemination of these valuable disaggregate data to a wider audience.

This report offers an in-depth exploration of this overarching tradeoff by:

- Examining the state of the literature with respect to geographic perturbation methods, disclosure risk and compromised data utility.

- Developing a conceptual framework to guide efforts of geo-perturbation with respect to risk disclosure and data utility.

- Conducting an empirical application of an innovative methodology that has the potential to permit the dissemination of higher resolution household travel survey data that also protects the confidentiality of the respondent's household location. 
- Quantifying the concepts of disclosure risk and data utility in this implementation of a geographic perturbation technique in order to improve the understanding of their tradeoff.

In addressing these objectives, this report begin with a literature review describing the present knowledge base regarding geographic perturbation methods, strategies for evaluating disclosure risk and data utility, and how these disaggregate data have been used to inform research on travel behavior and the built environment. This review is followed by the overview of a conceptual framework for assessing the fundamental concepts of disclosure risk and data utility. A methodological strategy for evaluating these ideas is then put forward, which is followed by a description of the results from an empirical application of this geographic perturbation technique. This report concludes with the identification of general guidelines for future applications as well as a greater discussion of methodological limitations and directions for expansion. 


\subsection{LITERATURE REVIEW}

Disaggregate data sources are favored for modeling the spatial link between household travel behavior and the built environment (Cervero and Kockelman, 1997; Krizek, 2003). Accordingly, research on this link often employs survey data specific to an individual's residence, introducing a set of complications centered on protecting the participant's confidentiality while ensuring the possibility of valid geographic analyses (Armstrong et al., 1999). Though largely absent in travel behavior research, the negotiation of this balance has recently been studied in public health research (Young et al., 2009) through the use of geographic masking processes in which precisely geocoded locations are modified to an extent considered sufficient for these data to be released to external users (Rushton et al., 2008). These masking processes differ from the aspatial approaches toward preserving statistical confidentiality, which have received considerable attention in the literature (Leitner and Curtis, 2006), by placing added consideration on providing spatial confidentiality through masking the spatial placement of an individual's information.

The masking of spatial data seeks to minimize the risk of disclosure or any breach in confidentiality that allows an outsider to discern either the identity of an individual or her associated attributes being reported under a pledge of confidentiality (Gutmann et al., 2008). The former breach, related to when the disclosure of a specific respondent enables a direct association to a record, is termed identity disclosure; while the latter breach, attribute disclosure, occurs when potentially sensitive information about an individual is disclosed as a result of linking a record to the respondent (Duncan and Lambert, 1989). A third concept of disclosure, referred to as inferential disclosure, occurs when the data user is able to infer new information about a respondent despite the released data being inexact and unassociated with the respondent's record (Dalenius et al., 1982). Inferential disclosure is related to the geographical differencing problem, where confidential information may be gleaned by an outside user through the subtraction of data presented at multiple, independently safe, non-coterminous geographies (Duke-Williams and Rees, 1998). Each disclosure type has an intrinsic risk that the data custodian will experience as a consequence of releasing any particular data source (Domingo-Ferrer and Torra, 2004). This disclosure risk must be weighted by the data releaser against the complementary concept of data utility, which measures the value of the released data source to the legitimate data user. In general, decreasing the amount of disclosure risk by applying more stringent geographic masking processes also decreases the accuracy of inferences obtainable from the released data source (Karr et al., 2006). While there is a wide understanding of this tradeoff between disclosure risk and data utility, there is no consensus on a particular methodology to visualize and share confidential data without dramatically limiting any analyses (Curtis et al., 2011).

\subsection{GEOGRAPHIC PERTURBATION METHODS}

Traditionally, the most common geographic masking technique has been to conceal individual records by aggregating the records (Rushton et al., 2008). One aggregation approach, areal aggregation, enumerates the total existing within a predefined political or administrative entity, whereas a second approach, point aggregation, assigns individual records to a single location representing a subset of the original locations (Armstrong et al., 1999). The ubiquitousness of this 
masking method is likely attributed to the ease for local data custodians, who may be technically or resource limited, to conduct this technique (Curtis et al., 2011). Armstrong et al. denoted four means in which the ability of the researcher to detect clusters or investigate potential relationships is compromised by employing aggregation (Armstrong et al., 1999). These data utility shortcomings of aggregation include: the absolute and relative locations of the individuals becoming unobservable in the geographic extent; the detectable size of clusters being limited by the selected aggregation scale; the requisite of spatial conformity also limiting cluster detection; and the geographic mismatch of collecting built environment data at a more disaggregate geography. Moreover, Rushton et al. (2004) note bias introduction when employing areal aggregation since these political or administrative entities are typically heterogeneous in terms of population density and demographics. Ultimately, the reduction in spatial resolution related to aggregation masking diminishes the data utility of the information (Kwan et al., 2004).

A second set of geographical masking techniques reflect affine point transformations in which data points are deterministically repositioned to a new set of locations (Domingo-Ferrer and Torra, 2004). One affine point transformation approach, translation, laterally shifts data points a determined distance and direction from their original location while preserving geographic scale (Kwan et al., 2004). Another affine point transformation method alters each data point by a specified scaling constant that is multiplied to each of the geographic coordinates of the original data point (Leitner and Curtis, 2006). This scale change technique differs from the translation technique because of its additional distortion of the distance between original point locations (Armstrong et al., 1999). A final method of affine point transformation is conducted by rotating the original spatial position of an individual record by a fixed angle (Armstrong et al., 1999). Instead of rotating the data points by using the original location as a pivot, the analyst may choose to translate the original data points before the rotation so as to pivot from an arbitrary location (Leitner and Curtis, 2006). Correspondingly, an affine transformation approach may be performed that utilizes any combination of these three methods of translation, scaling change, or rotation (Kwan et al., 2004). Also, a stochastic component may be introduced to affine transformations, where the randomly chosen distance and direction of displacement for relative locations would remain preserved (Leitner and Curtis, 2006).

A third classification of geographical masking methods, random spatial perturbation, provides additional protection against disclosure by randomly selecting the displacement distance and direction for each repositioned individual record (Armstrong et al., 1999). The random spatial perturbation geographic masking technique has recently been referred to as jittering (French and Wand, 2004). Kwan, Casas, and Schmitz (2004) distinguish three subclasses of jittered data points in which the masked location is either randomly located along or inside the perimeter of a circle with a center at the original location and a chosen radius, or the masked location lies randomly within any other polygon defined relative to the original point. Such jittering methods may be employed by taking into account the population density surrounding the respondent's location by skewing her address by a random offset based on a Gaussian distribution whose standard deviations are inversely correlated to the population density (Cassa et al., 2006). This approach displaces individuals residing in an area with a lower population density by larger amounts than their counterparts residing in urban areas characterized by a higher population density since the former individuals have a heightened disclosure risk (Rushton et al., 2008). One promising adaptive random perturbation technique ensures that a masked record is moved a minimum 
distance from its original location, so the perturbed position is not randomly assigned on or near its original location (Allshouse et al., 2010). This donut masking technique has been shown to greatly improve disclosure risk with a negligible impact on the specificity and sensitivity of detecting clustering patterns or data trends (Hampton et al., 2010). As with all jittering techniques, the value of the radii should be carefully selected since the greater the distance from the original location to the masked location, the greater the pattern attributed to the original data deviates (Kwan et al., 2004; Hampton et al., 2010).

Additional geographic perturbation methods denoted in the literature include nearest-neighbor masking and geographic masking with contextual information (Armstrong et al., 1999). In the nearest-neighbor masking method, only the distance between an individual record and the nearest record(s) of interest is reported, which is often sufficient for clustering analyses (Rushton, 2006). Furthermore, certain cluster analyses (e.g., Cuzick-Edwards statistic) also require attribute knowledge of the nearest neighbor (Leitner and Curtis, 2006). To ensure confidentiality, no additional information aside from the distances to, and identity of, the two nearest neighbors exhibiting a particular attribute may be provided since it may be possible for an outside data user to detect the existing pattern and recreate the relative locations (Armstrong et al., 1999). In contrast, the contextual information method removes any geographic identification from the dataset and substitutes it with contextual information that is of interest to the data user (Gutmann, 2008). Analysis of the contextual information method has found that the use of smaller geographies for gleaning the contextual information presents an inappropriately high potential for disclosure risk (Armstrong et al., 1999).

\subsection{EVALUATION OF GEOGRAPHIC PERTURBATION METHODS}

As previously mentioned, the protection of anonymity, along with the preservation of information and structure, are two elements of importance to a user of data provided under a pledge of confidentiality. Similar to the lack of agreement on which geographic masking technique for the data custodian to employ, there is also no consensus regarding the amount of disclosure risk that is deemed acceptable (VanWey et al., 2005) and, accordingly, there are a number of statistical approaches to quantify any such risk disclosure (Leitner and Curtis, 2006). One such confidentiality measure, k-anonymity, is a special case of k-map protection where security is provided to ensure that released information may only be mapped to k incorrect entities (Sweeney, 2002). In terms of spatial representation, k-anonymity refers to the number of households whose jittered position is closer to the original location than the distance of displacement attributed to the geographic masking technique (Allshouse et al., 2010). Another statistical approach is Spruill's measure in which the squared distance between the masked location and original location is calculated and then the percentage of masked records closer to the original location than any other of the original locations is computed. This percentage of the geographically masked locations found to lie nearest to their original location is next multiplied by the sampling fraction to determine the degree of disclosure risk (Duncan and Lambert, 1989). A third broadly employed method for evaluating confidentiality preservation is to measure the area of a confidence region, covering a specified range of probability, for the true location of a spatially perturbed location (Rushton et al., 2008; Zimmerman and Pavlik, 2008). Zimmerman and Pavlik's research has employed this measure for disclosure risk in scenarios where multiple geographical masks have been applied to the original dataset. Finally, Rushton et al. (2008) denote a number of lesser- 
utilized methods for measuring disclosure risk that include a measure of vulnerability to local geographic knowledge and a measure detailing the minimum number of masked locations, which may be linked to the original dataset by obvious identifying attributes that would compromise the entire geographically masked dataset.

As a complement to minimizing disclosure risk, the data custodian must also attempt to preserve the utility of the content associated with the geographically masked data as well as the relationships between the point data and the resource characteristics of it surrounding spatial landscape. The challenge being that the data custodian is usually not in a position to predict the needs of the future data user and, accordingly, may need to examine the preservation capabilities of the data along several dimensions (Armstrong et al., 1999). One approach to be considered by the data custodian is the calculation of test statistics to determine whether the statistically significant spatial attributes in the original dataset remain significant in the perturbed dataset (Leitner and Buttenfield, 2000). A second approach is to examine whether the masked data preserves the distances and directions to relevant geographic features that were initially observed in the original point data (Armstrong et al., 1999). Another set of methods commonly used to examine the preservation of data utility includes point-pattern analyses such as the kernel estimation of density surfaces, cross-K functions, or more informal visualizations of clustering patterns (Kwan et al., 2004; Olson et al., 2006). The location of these clusters is often more important than the mere existence of spatial clustering (Armstrong et al., 1999). Finally, similar to the detection of spatial clustering, the data custodian may also anticipate the necessity to maintain any monotonic trends, and their directionality, in the masked dataset that were exhibited in the original point data (Armstrong et al., 1999). Ultimately, the data custodian must carefully consider the concept of data utility, as each geographic perturbation technique results in the loss of some original spatial information to the data user and some approaches preserve more valuable information than others (Rushton et al., 2008).

\subsection{TRAVEL BEHAVIOR AND THE BUILT ENVIRONMENT}

Guiding this research is the desire to put forth a geographic perturbation method that permits the confidential release of valuable disaggregate household data, which will in turn improve the quality of research into the connection between household travel behavior and the built environment. The past inability to release such data has been an obstacle toward an improved understanding of the linkages that exist between travel behavior and the built environment because these disaggregate data are most suitable to study since they circumvent the methodological concern of an ecological fallacy (Handy et al., 2002). Accordingly, past studies of the relationship between household travel patterns and the built environment have been suspect of any inferential comparison of the disaggregate housing unit to a more aggregate representation of neighborhood (Goulias and Kim, 2001; Bhat and Zhao, 2002) and have confirmed the inherent risk of aggregating household data into the zones traditionally exhibited in four-step travel demand models (Chikaraishi et al., 2009). To avoid this modeling pitfall, travel behavior research has continued to advance in the direction of employing activity-based travel demand models that rely extensively on disaggregate built environment and socioeconomic measures in order to properly capture their effects on observed household travel (Badoe and Miller, 2000; Davidson et al., 2007). Understanding these relationships has become ever more imperative as regional travel demand models continue to increasingly account for non-motorized travel modes (Rodriguez and Joo, 2004). 
Previous literature on the connection between travel behavior and the built environment has been systematically reviewed by classifying the latter element as "D" variables (Ewing and Cervero, 2010). While a seemingly infinite number of built environment measures may be considered for any research into the transportation-land use link, this research has chosen to focus on a handful of measures related to density, design and distance to transit. In terms of population density and non-motorized travel, Frank and Pivo (1994) found that walking trips were most sensitive to increases in population density at the trip origin. Greenwald and Boarnet (2001) found that population density had a significant connection to walking for discretionary trip purposes when operationalized at the census block group, but not when measured at the more regional ZIP code boundary. Often used as a complement to population density, Cervero (2006) found increased employment density reduced the odds of vehicle ownership. An and Chen (2007) found employment density, when reflected at the census block group, had the strongest power in predicting non-motorized mode shares. Intersection density, a design measure that represents opportunity for path choice, was found by Schlossberg et al. (2006) to be a significant predictor of walking for school trips, which echoed the link found in past studies that note this measure of network connectivity to be a significant predictor of walking (Frank et al., 2008). Aside from built environment measures of density and design, past travel behavior research has also focused on accessibility measures examining the distance to the nearest bus stop or rail station. In regard to distance to the nearest bus stop, Targa and Clifton (2005) found that individuals residing closer to a bus stop tended to generate more walking trips, while Susilo et al. (2012) echoed past research by finding that a shorter distance to the nearest bus stop promotes public transport use. As for distance to the nearest rail station, Chatman (2009) found that residents living within one-half mile of a heavy rail station significantly conducted more discretionary transit trips, while Chen et al. (2008) found that long distances to public transit stations increased the propensity to drive for home-based work tours. 


\subsection{THEORETICAL FRAMEWORK FOR DISCLOSURE RISK AND DATA UTILITY}

Understanding that household travel surveys inherently provide characteristics of the individual or their household unit to the data collector and as such make any complete avoidance of disclosure risk all but impossible, great importance is placed upon the data custodian to control or limit the potential of any breach in the confidentiality pledge between the survey respondent and data collector. However, there is no general agreement on what constitutes a tolerable level of disclosure risk or how to sufficiently guarantee the anonymity of the survey respondent. Moreover, disclosure risk may not always be related to a breach in confidentiality caused by the illegitimate use of the geographically explicit dataset to infer confidential individual information. Such an exception is termed inferential disclosure (Duncan and Lambert, 1989) and occurs when the release of a dataset leads to the potential to disclose confidential information about an individual who is not the survey respondent. While out of this report's scope, which is inferential, attacks and data intruder behavior have been discussed in past literature on the topic (Paass, 1988; Fuller, 1993). Instead, this report is centered on the principle that public and private agencies must consider disclosure risk from the perspective of both the data intruder and legitimate data user in order to effectively evaluate the competing concepts of disclosure risk and data utility for different transportation-land use applications.

Assessing the complex tradeoffs between disclosure risk and data utility has proven to be largely heuristic and may be based on any number of assumptions and scenarios. Thus, a theoretical framework that provides a coherent and consistent method for evaluating these tradeoffs is essential toward any study aimed at providing an implementable methodology. The proceeding discussion provides a conceptual framework, separated into three steps, to consider when developing a methodological approach for releasing spatially sensitive household travel survey data. In the first step in this proposed framework, a hierarchical evaluation of disclosure risk is performed in which the researcher considers what records ought to be geographically perturbed. Having given ample consideration toward minimizing disclosure risk in this first step, the researcher then addresses the complementary concept of data utility by noting which data are most valuable for the analysis of interest and therefore require a higher level of spatial resolution. Finally, the third step balances these competing concepts of disclosure risk and data utility in the selection of an appropriate geographic perturbation method. The objective of this last step in the theoretical framework is to minimize the potential of disclosure risk that was determined in the first step, while deciding on the maximum tolerance for introduced spatial error to the original dataset that is considered in the second step.

\subsection{EVALUATION OF DISCLOSURE RISK}

Disclosure in the context of this report is understood to be the identification of a sample record in a released dataset that has occurred without any prior knowledge of the survey respondent being part of the sampled dataset and the recognition of additional attributes of the survey respondent (Fellegi, 1972). Identity and attribute disclosure risk were discussed in the literature review and are reflected in the three-tiered hierarchical framework for evaluating confidentiality disclosure illustrated in Figure 3-1 (Clifton and Noyan, 2012). The divisions of disclosure risk into separate 
tiers within this framework distinguishes the likelihood of identity and attribute disclosure occurring as well as the type of additional information required by the data intruder to extract such confidential data. Such an evaluation of disclosure risk requires certain assumptions about the goals of the data intruder to be established by the individual responsible for disseminating the data source. By establishing assumptions about the motivation and state of knowledge characterizing the data intruder, this report puts forward a standard approach for conducting a hierarchical evaluation of information tiers with respect to disclosure risk. Intuitively, this hierarchical framework suggests that the geographic perturbation of the original dataset must increase as the data custodian moves down the tiers of the disclosure risk hierarchy. A greater discussion of each of these three tiers is provided in the following paragraphs.

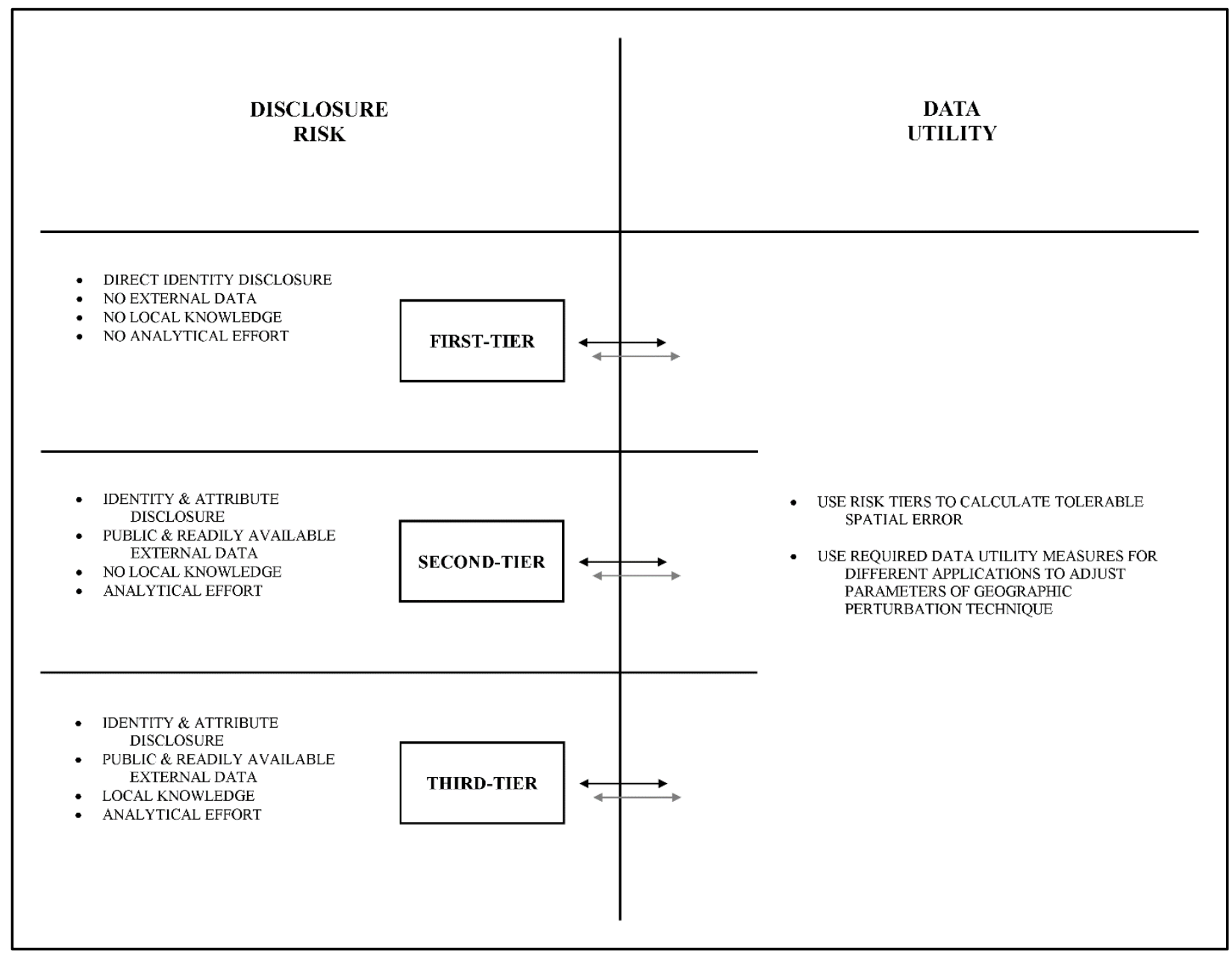

Figure 3-1: Hierarchical framework of disclosure risk and data utility

The first tier of the hierarchical evaluation framework of disclosure risk reflects the release of information that provides the data intruder with the most obviously identifiable information about the survey respondent. This type of disclosed information, referred to as a direct identifier, provides the data intruder with the ability to directly associate an individual, household or workplace to a sampled record within a publicly released dataset. This highest level of disclosure risk is the realization of this one-to-one correspondence; however, the assignment of such a risk does not completely depend on the level of disclosure risk, but also the degree of analytical effort 
required by the data intruder to make this direct association. The realization of this one-to-one correspondence between the perturbed sample record and the identity of the survey respondent without any external information or additional effort from the data intruder is classified as a firsttier risk and the worst degree of disclosure risk. Such records require an added level of data masking, which may be achieved through the application of a geographic perturbation method, to ensure these flagged sample records are provided with an ample amount of anonymity.

However, after the first-tier analysis is completed on the household travel survey dataset by the data custodian, sensitive information about the survey respondent that is inferable by a data intruder may remain. The second tier of analysis within the hierarchical evaluation framework begins to address this possibility of identity and attribute disclosure that may occur with added effort by the data intruder using supplemental data sources. In this second tier, the data custodian must be cognizant of more subtle disclosure risks within the collected dataset that arise because of sample size or the existence of unique sampled records. Moreover, the data custodian must now consider a series of assumptions that pertain to the possible familiarity of the data intruder with elements of the dataset and their intention for establishing any direct one-to-one correspondence. One such assumption is that the data intruder may be willing to use external, publicly available data sources to disclose the identity or attributes of the respondent.

This level of disclosure risk in which the potential exists for a data intruder to disclose the identity and attributes of the survey respondent through the complementary use of easily accessible public datasets, such as those provided by the U.S. Census, is termed second-tier risk. While this level of risk analysis does not assume any local knowledge by the data intruder of the survey respondents or study area, there is an assumption that the data intruder will use common identifiers such as income, race or household size to increase the prospects of a direct correspondence between the perturbed sample record and actual respondent. The motivation of the data intruder and analytical skill set is greater in this second level of disclosure risk and, accordingly, the data custodian must place greater thought toward ensuring respondent confidentiality.

For instance, the data custodian who is evaluating this second-tier disclosure risk should place greater attention on any geographic identifiers detailed in the collected dataset. Information for similar geographies such as census blocks, while likely independently safe, may be spatially compared to attain distributional characteristics and reveal information to the public that was unintended. One desired result of the spatial aggregation of census data is the difficulty of making any direct correspondence that would jeopardize respondent confidentiality. Yet, there still exists the potential to link the behaviors of similar populations or at the very least discard more unlikely hypotheses. For unique observations within the collected dataset, uncovering general trends about the individual's census block may provide the extra information needed by the data intruder to reveal the individual's identity and attributes. It is at this step that the data custodian should conduct an extensive review of widely available datasets to the public that may be utilized to infer variations in socio-demographic or built environment attributes across the geographic entities at which survey respondents are allocated.

Undoubtedly, any review process requires greater effort by the data custodian and also raises questions regarding the temporal and substantive relevance of identified datasets. Within the former tier of the hierarchical framework the data custodian only needed to be aware of trends 
within their collected dataset; however, in a second-tier analysis, the data custodian is now required to understand historical and general growth patterns across the geographies comprising the study area. While the growth rates and patterns across a more urban context are more likely to be more dynamic, the same trends revealed for a rural context may experience little variation over longer stretches of time. In such instances, certain data from an older resource may continue to inform the perturbation decisions of the data custodian, while other data may have become temporally irrelevant.

Aside from the decision of whether the relevance of identified data sources has expired, the data custodian evaluating within this second tier of disclosure risk must also contemplate the substance or accuracy reported within these external datasets. Datasets vary in regard to the accuracy levels of their collected information, thus the data custodian must understand how these accuracy levels differ from their collected dataset in terms of reported variables and geographies. As with the temporal discussion, outliers must be given greater attention when examining the accuracy of secondary data sources since these unique records are more susceptible to disclosure risk. The likelihood of disclosure increases with improved geographic accuracy since a reduction in the number of records within an aggregated geography will reduce the screening of a unique record from the pool of respondents. This occurrence may create a serious consequence since it could lead to the identification of a unique record that may be highly valued in the dataset due to its uncommon attributes. Comparing the survey data against all temporally relevant public datasets will help to inform what sampled records may require greater geographic displacement in order to avoid a second-tiered risk of disclosure.

Similar to middle tier in the hierarchical framework for disclosure risk, the final tier calls for the geographic perturbation of the survey dataset due to increased potential for identity and attribute disclosure as a result of a data intruder incorporating external secondary sources. A main difference between the second and third tiers is the assumption made by the data custodian in the third tier that the data intruder has some local knowledge or experience. The assignment of a third-tier disclosure risk to a record denotes that any inference depends on not only a finer level of knowledge and experience, but also a heightened analytical skill set and investigative effort by the data intruder. The evaluation of a second-tier risk centered on ensuring that anonymity was maintained in regard to commonly recorded socio-demographic and economic characteristics, but a third-tier analysis must also account for those attributes disclosed by the survey respondent that necessitate more specific knowledge about the respondent or her community in order to establish a one-to-one correspondence. This local intimate knowledge of a survey respondent, which may include individual attributes such as typical commute mode choice, is not deemed essential to guard against in this hierarchical framework, but should be considered and evaluated by the data custodian prior to any public dissemination of the dataset. This is due to a number of reasons including the difficulty in understanding what assumptions to make about a data intruder's local knowledge and the increased potential of a third-tiered risk occurring after a dataset has already been made public. Any best effort by a data custodian to avoid the identity and attribute disclosure of a survey respondent may be weakened - despite careful consideration of any number of assumptions about the data intruder's knowledge and effort - by the future release of a secondary dataset. 


\subsection{EVALUATION OF DATA UTILITY}

The assessment of data utility, which describes the value of the geographically perturbed information to the researcher, balances the previous consideration of disclosure risk. After selecting an acceptable level of disclosure risk to protect the confidentiality of the survey respondent based on the aforementioned hierarchy, the data custodian must then consider the usefulness of the geographically perturbed dataset to the researcher. Similar to the hierarchical evaluation of disclosure risk, this second step within the conceptual framework poses several challenges to the data custodian revolving around the degree of resolution needed for a specific application and the selection of an acceptable level of introduced spatial error. Also, the data custodian is likely unaware of all potential future applications of the perturbed dataset at the time of its release, scenarios in which the researcher would like to have greater spatial resolution or less error. As such, this evaluation of data utility may be more appropriately thought of as a second step within an iterative process rather than as an independent step following the completion of any disclosure risk evaluation.

Estimating the utility of a geographically perturbed dataset is reliant on attributes of the survey respondents that are of interest to the researcher. Accordingly, while data utility may be thought of in terms of the overall collection of attributes found in a released dataset, considerable variation exists across individual records and the attributes describing these sampled records. Thus, when assessing the utility of a perturbed dataset, each attribute must be evaluated based upon its distribution within the sample as well as the composition of the attribute. Whether the variable is continuous or discrete in nature, and how the variable may be compared to other collected attributes of the individual, must also be considered within this step. At the very least, the data custodian must consider these aspects of data utility at a spatial resolution suitable to the majority of applications the collected dataset was envisioned to inform. This helps to understand the direct and complex relationships between better-quality resolution and an acceptable tolerance for introduced spatial error.

Since this second step in the theoretical framework is difficult to describe in the abstract, two potential applications for implementing a geographic perturbation method to household travel surveys are discussed in the following paragraphs to better ground this discussion of data utility evaluation. For instance, household travel surveys commonly ask a respondent to approximate her annual household income in order to use the information in a host of transportation planning applications, such as trip-generation models. In this illustration, one application of a tripgeneration model may estimate the number of trips conducted by an individual or household with a regression equation where income is represented as a continuous attribute and where the tolerable error depends on the sensitivity of the estimated regression model to household income. Another trip-generation application may utilize a cross classification method relating average individual or household trip rates to a discrete number of income brackets. The aggregation of household income in the latter trip-generation models is less sensitive to the introduction of spatial error than the former application, which would be less tolerable to introduced error in the dataset.

In addition to the estimation of trip-generation models, data from household travel surveys are being more frequently used to examine the relationship between non-motorized mode choice and a household's surrounding built environment. In the past, travel demand models have been almost 
solely interested in examining automotive travel at a more aggregated geographic scale; however, advances in travel demand modeling techniques interested in alternative travel modes have powered the need for releasing disaggregate trip-end representations. Previous transportation models that represented the household unit at aggregate spatial scales such as a traffic analysis zones or census geographies were more tolerable of greater errors in travel time and distance. When examining alternative travel modes, especially non-motorized modes, this aggregate geographic scale becomes less suitable due to shorter travel distances that lead to a disproportionate level of intra-zonal trips. However, as with the release of a continuous representation of household income in lieu of a categorical representation, the improved utility of a more disaggregate dataset, specifying the spatial location of the trip origin and destination, also brings a heightened prospect of disclosure risk. Surprisingly, there exists a dearth in transportation literature objectively examining the geographic extent most appropriate for studying the relationship between non-motorized mode choice and the built environment. This limitation adds to the difficulty in defining an acceptable tolerance for introduced spatial error. Yet, one may imagine the output of a transportation model estimating ridership originating at a specific bus stop or rail station may be biased if household locations are allowed to be geographically perturbed a distance beyond what is deemed to be accessible to pedestrians.

The above examples illustrate the complexity of evaluating data utility in comparison to its counterpart of disclosure risk. Not only are transportation agencies less likely to predict all of the potential uses of their collected datasets, but researchers have also limited their understanding of what levels of introduced error are acceptable in more recent applications. Given these present shortcomings, the data custodian may defer any long evaluation into preserving data utility after the completion of the final step in this theoretical framework.

\subsection{IMPLEMENTATION OF A GEOGRAPHIC PERTURBATION TECHNIQUE}

In an ideal succession through the conceptual framework, at this point, the data custodian will have evaluated both disclosure risk and data utility so as to provide anonymity to the survey respondent and produce the most useful dataset to the researcher's application. Having struck some balance between the objectives of minimizing disclosure risk and maximizing data utility, or simply settling on a required degree of anonymity to enable the dataset's public release, the data custodian will then select the most appropriate geographic perturbation technique. The prior literature review described the three common classes of geographic perturbation techniques that may be applied to the dataset by the data custodian: aggregation, affine point transformation, and random geographic perturbation. The focus of this final step in the theoretical framework is to explore these different methodologies in the context of (1) the overall tradeoffs of perturbing the dataset in regard to the disclosure risk and data utility, and (2) the effectiveness of different geographic perturbation techniques at protecting personal privacy and providing accurate analytical results. In the end, the application of a specific geographic perturbation technique will be determined by the selected risk tier and intended application of the collected dataset.

By implementing an aggregation method to geographically perturb the original dataset, whether through a zonal or point technique, the data custodian is selecting a strategy that will likely result in an imbalance between disclosure risk and data utility. The application of an aggregation method, 
as found in past travel demand models representing activity at the traffic analysis zone, is likely to provide an adequate level of anonymity to mask the survey respondent from detection by a data intruder, but at the tradeoff of limited data utility. As previously mentioned, the representation of travel survey data at a large geographic scale hinders the ability of the analyst interested in nonmotorized travel to draw many meaningful conclusions. This unevenness that places more emphasis on minimizing disclosure risk than maximizing data utility reflected by the use of an aggregation method is shifted when applying an affine point transformation to the original dataset. In an affine point transformation, the data utility is generally improved due to the finer resolution associated with the disaggregate representation of the record; however, the deterministic positioning of the geographically perturbed records increases the risk of the respondent having her confidentiality compromised. The randomization in the positioning of the disaggregate sample record, which characterizes the third class of geographic perturbation methods, enables the data custodian to provide both the resolution necessary for maximizing data utility as well as the anonymity necessary for minimizing disclosure risk. While the random spatial perturbation gives the data custodian the opportunity to balance disclosure risk and data utility, the implementation of this geographic perturbation method requires a strong understanding by the data custodian of how these two themes interact across different techniques and applications of the dataset.

For common applications, such as the use of household travel survey data for regional travel demand modeling, the data custodian may provide information to the researcher describing the level of anonymity and error introduced to the geographically perturbed dataset. Information on the former theme would provide the agency with an idea of what the minimum level of anonymity is for records in the spatially perturbed dataset, whereas measures of the error or confidence intervals would provide the agency a sense about the latter theme. Given that agencies will have both the original and spatially manipulated datasets in their possession, an analyst may then be able to evaluate the impact that the use of a selected geographical perturbation technique will have on the intended application of the data. Since the range of potential uses for such data is unknown, and probably incompletely understood by the agency at the time of receiving the processed dataset, it is best for the agency to examine the best compromise of disclosure risk and data utility whenever a new application of the data is considered. In this sense, the evaluation of disclosure risk and data utility becomes an ongoing exercise dependent on the selection of geographic perturbation technique and application of the collected dataset. However, given that resources are limited, the evaluation of the scenario with the lowest risk of disclosure that produces an acceptable amount of error seems sufficient for common or foreseeable applications. 


\subsection{METHODOLOGY}

In an attempt to empirically test the above theoretical framework, the remainder of this report explores the application of one promising geographic perturbation technique aimed toward reducing the risk of identity disclosure while maintaining data utility. This particular application of a random geographic perturbation technique has been guided by the desire to avoid a first-tier risk of identity disclosure and the complementary motivation of quantifying how an increased level of survey respondent anonymity relates to the utility of the dataset. Specifically, this methodology is designed to provide information on how the random geographic perturbation of a survey respondent's household relates to the utility of a selection of built environment measures commonly estimated in models exploring the linkage between non-motorized travel and the built environment. The following discussion will describe the technique selected for this empirical application, the statistical measures used to evaluate disclosure risk and data utility, and the datasets used for this particular implementation.

\subsection{RANDOM GEOGRAPHIC PERTURBATION TECHNIQUE}

Directed by the increasing desire of transportation researchers for a wider dissemination of household travel survey data that represents the unit of analysis at a finer geographic resolution and the inability of agencies to publicly release these disaggregate data for fear of compromising the confidentiality pledge established between the respondent and survey administrator, this research examines the empirical implementation of an innovative geographic perturbation technique. Conceptually, this application of the selected technique begins with the geographic identification of the survey respondent's household and the construction of a circular buffer extending outward from the respondent's household location. The perimeter of this first circle represents the minimum distance that the geographically perturbed household must be repositioned in order to adequately ensure the household has a sufficient level of anonymity to prevent a oneto-one correspondence and subsequent identity disclosure of the survey respondent.

The established circle represents an exclusion area in which the geographically perturbed household cannot be positioned within. Next, the data custodian constructs a second circular buffer that also extends outward from the original household location. The perimeter of this second circle represents the maximum displacement distance that the survey respondent's household location may eventually be geographically perturbed. Meanwhile, the area of this circle represents the displacement area that the household location will be repositioned. Similar to the first buffer, the data custodian is responsible for the selection of the radius of this second circular buffer around the household location, which will help inform the utility of the altered dataset. Completion of this latest step generates a torus or donut-shaped area with the original household located at the center, which represents the potential area that a household may now be randomly repositioned. Figure 41 provides an illustration outlining the general steps taken toward implementing this random geographic perturbation procedure. The donut masking technique, which has not been previously examined in transportation-related research, was only recently introduced as a promising random geographic perturbation technique in the field of public health research (Allshouse et al., 2010; Hampton et al., 2010). 
STEP 1:

Locate Actual Household in

United States Census Tract

STEP 2:

Specify K-Anonymity Statistic to Create

Inner-Ring and Exclusion Area

STEP 3:

Specify Outer-Ring Radius to Define

Donut for Scenario
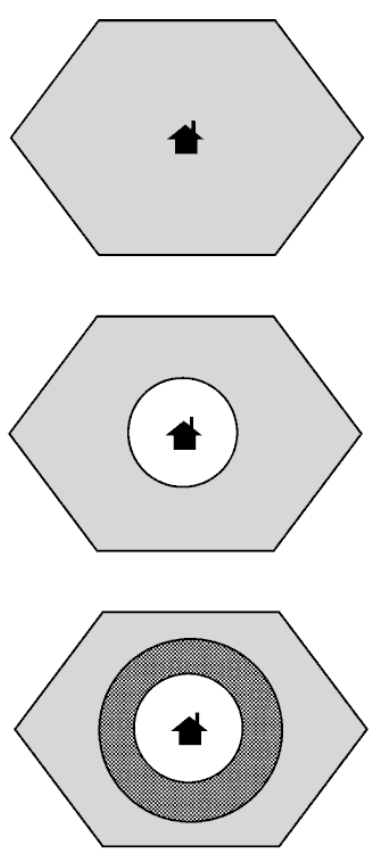

STEP 4:

Randomly Reposition Actual Household

within Donut

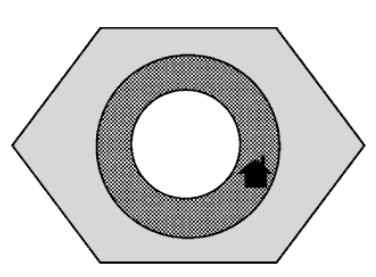

Figure 4-1: Conceptual steps in applying the donut masking technique

Provided with this conceptual description of how the donut masking technique may be applied to a household travel survey dataset, a number of questions arise concerning the selection of an innerand outer-ring radius. Considerable attention must be given by the data custodian in the selection of an inner-ring radius that provides a sufficient level of anonymity as well as an outer-ring radius that ensures only an acceptable amount of spatial error is introduced in the geographically perturbed dataset. While the selection of these separate radii appears somewhat subjective in nature, the data custodian must be able to somehow quantitatively justify the selection of these distances bounding the donut-shaped area. In order to achieve this objective within this application, the selection of an inner-ring radius has been linked to a statistical measure of anonymity found in the information sciences, while the selection of an outer-ring radius is informed by general rules of thumb found in transportation planning research.

As noted in the literature review, there have been a variety of statistical methods explored by researchers in an attempt to accurately quantify the level of anonymity that a geographically perturbed household has been provided. Instinctively, the anonymity of a survey respondent in this geographic context is a function of the underlying population density of the area surrounding the individual's spatial location. Thus, any statistical reflection of anonymity in a geographic perturbation technique must be directly linked to the number of additional individuals or 
households found within the exclusion area of the donut. By utilizing the k-anonymity concept introduced by Sweeney (2002), where $\mathrm{k}$ is a value representing the total number of households, the original household location cannot be reversely identified (Cassa et al., 2006). Allshouse et al. (2010) provided the following operationalization of this concept of anonymity, which may be rearranged to reflect an inner-ring radius for the donut masking technique informed by the selection of a k-anonymity level by the data custodian.

$$
\hat{k}=\pi \times d^{2} \times\left(\frac{N}{\text { area }}\right)
$$

In the above equation, the estimated k-anonymity statistics is equal to the product of pi and the square of the straight-line distance from the household location, $d$, multiplied by the number of occupied households within the survey respondent's census tract, $N$, divided by the land area of the household's census tract. The use of census tract as the geographical representation of the household's surrounding population adds a restriction on the exclusion area where the geographically perturbed location of the household must remain within the original census tract. Hampton et al. (2010) described this strategy in applying the donut masking technique as well as a second approach in which the spatially perturbed household is allowed to be positioned outside of its original census tract. The implementation of the latter strategy would be suitable if the data custodian has access to a disaggregate representation of the population; however, since such data are not widely available, the data custodian may be more likely to select the former strategy. In the restricted approach, which was selected for this exploration, the choice of a higher k-anonymity statistic may lead to a scenario where the exclusion area is not circular in shape due to the household being located in proximity to the boundary of a census tract.

As a complement to the choice of an inner-ring radius and its relationship with the minimization of disclosure risk is the decision of an outer-ring radius and its relationship with the maximization of data utility. In this implementation of the donut masking technique, the choice of an outer-ring radius represents the maximum distance a household may be repositioned from its original geographic site. In general, the choice of a greater outer-ring radius has a direct connection with the acceptance of a greater amount of spatial error being introduced to the geographically perturbed dataset. Accordingly, the data custodian must consider the amount of spatial error that can be introduced to the dataset given the intended application of the data. Also, as the choice of an initial threshold for displacement distance is somewhat arbitrary since no evaluation of data utility may be conducted until the household has been geographically perturbed, this decision of an outer-ring radius should also be guided by theory or common practice. In studying the link between nonautomotive travel and the built environment, researchers often employ straight-line buffers extending from the household's physical location (Clifton et al., 2009), which are drawn at a distance covering the range that an individual would likely consider when walking. A number of studies have examined the built environment within one-half mile or one mile areal buffers (Krizek, 2003; McGinn et al., 2007), while others have looked at the built environment within smaller one-quarter mile buffers (Lee and Mouden, 2006) or larger one and one-half mile buffers (Schlossberg, 2006). Consequently, the application of these distances for the outer-ring radius may be seen as justifiable choices. 
Additionally, the data custodian may wish to select an outer-ring radius that is greater in length when considering more rural settings. Reasons supporting this consideration may include either the perception that the built environment does not vary as much across rural contexts in comparison to more urban areas or the potential of the inner-ring radius exceeding the outer-ring radius. The latter condition must be investigated by the data custodian since the collapsing of the donut will result in the household not being geographically perturbed, but instead left in its original disaggregate position. Such an instance would produce an estimated k-anonymity statistic with a value of one and compromise the anonymity of the survey respondent by creating a one-to-one correspondence. This decision to be made by the data custodian underscores the importance of selecting an appropriate inner-ring and outer-ring radius since the two are not explicitly connected to one another. Finally, as with the creation of the exclusion area, the generation of the donut may also be inhibited by the boundary of the census tract if the chosen maximum displacement distance extends beyond the geographical unit in any particular direction. Similarly, the close proximity of any water feature may constrain the area of the donut since only land area is considered for a geographically perturbed household location in this application.

\subsection{QUANTIFYING DISCLOSURE RISK AND DATA UTILITY}

Having defined the inner-ring radius based on a chosen estimated k-anonymity statistic and the outer-ring by an accessible walking distance, the data custodian must now select a measure to quantify the concepts of disclosure risk and data utility. While the decision of what combination of radii is somewhat of a subjective effort, the quantitative expression of these two themes allows the data custodian to better inform the researcher of what level of anonymity and spatial error exists in the geographically perturbed dataset. Being apprised of these measures subsequently enables the researcher to decide whether these levels are appropriate for the specific type of application under consideration. For instance, a researcher may decide that the introduction of spatial error to a dataset describing the relationship between repositioned household locations and a specific measure of the built environment is above a threshold of her likening for a study into the link between non-motorized mode choice and the built environment. Therefore, the researcher may require an application of the donut masking technique to the original dataset that presents less spatial error. At this point, the data custodian must determine whether the release of a more precise dataset would compromise the anonymity of survey respondents from a data intruder. If not, then the researcher must reassess her intended use of the altered dataset.

In this methodology, a version of the estimated k-anonymity statistic used to define the inner-ring radius of the donut was also utilized to help express the potential for a data intruder to successfully make a one-to-one correspondence of the perturbed household location with the actual location of the survey respondent. Unlike the choice of a k-anonymity statistic in defining the exclusion area of the donut by stating the absolute minimum number of households that a geographically perturbed household could potentially be mistaken for, the use of k-anonymity in representing respondent confidentiality is a summary measure. The reason for this being a summary statistic is that the data custodian must try a number of simulations for spatially perturbing the original household location in order to better understand the overall behavior of anonymity within a selected trial of inner- and outer-ring sizes. When randomly repositioning a household within a donut resulting from some specified combination of inner- and outer-ring radii, a greater discrepancy between the two ring sizes will lead to a widening gap between the absolute minimum $\mathrm{k}$-anonymity and the average k-anonymity statistic. If the inner ring is held constant and the outer 
ring, which reflects the absolute maximum k-anonymity, is allowed to increase, then the range of potential k-anonymity values widens across the different simulations. As such, the selection of a higher number of simulations will better enable the data custodian to describe the distribution of k-anonymity statistics within a specified donut. Therefore, for this exploration of the donut masking technique, there were 50 simulations completed to describe the minimum, maximum, median, and mean k-anonymity statistic for a particular scenario. These measures inform the potential for identity disclosure risk in the produced geographically perturbed dataset.

To quantify the contending concept of data utility, the summary statistic of percent root mean square error (PRMSE) was calculated across the 50 simulations for a chosen combination of innerand outer-ring radii. This measure of spatial error was calculated using the following formulation.

$$
P R M S E=100 \times \sqrt{\frac{1}{N} \sum_{i=1}^{N} \frac{\left(x-\widehat{x}_{l}\right)^{2}}{\frac{1}{N} \sum_{i=1}^{N} x_{i}}}
$$

In the above equation, $x$ represents the value of a built environment measure in relation to the actual household location, whereas $x_{i}$ represents the value of the same built environment measure in relation to the geographically perturbed location of the household and $\mathrm{N}$ represents the number of simulations within each scenario. The PRMSE of a selected built environment measure is calculated for each sampled record in the spatially perturbed dataset with the estimated PRMSE values then being averaged across the entire sample to produce a single statistic to represent spatial error. Since the magnitude of built environment measures is likely to range across different contexts, the representation of spatial error with one such aggregate statistic may not provide sufficient information to the researcher about the degree of variation found within an altered dataset. Therefore, the data custodian may choose to calculate this expression of data utility across different classifications of the built environment measure of interest in order to provide the data researcher with a glimpse at how spatial error fluctuates in these discrete categories. The data custodian may decide to divide the average PRMSE of a built environment variable based on any number of schemes including natural breaks or manual classification.

\subsection{EMPIRICAL APPLICATION}

To further explore these representations of disclosure risk and data utility, an empirical application of the donut masking technique was conducted in the Portland metropolitan region. Figure 4-2 provides a map of the three-county study area covering Multnomah, Washington and Clackamas counties. Within this region, the connections between 4,824 households and five measures of the built environment were investigated. Data on the geographic locations of the households were provided by the Oregon Travel and Activity Survey, which was a household travel survey collected across Oregon from 2009-2011. Sampled households for the Portland metropolitan region were surveyed in 2011. All attributes of the survey respondents were removed in this empirical application, with the exception of the geographic coordinates of the household location.

The susceptibility of a surveyed household to identity disclosure risk was measured by utilizing population data from the 2010 Census at the tract geography. Since the unit of analysis in this 
particular application was the household unit as opposed to the individual, the field reflecting the number of occupied households within a census tract was used. Additionally, information pertaining to the land area of a census tract was supplied by the same data source to complete the calculation of the estimated k-anonymity statistic.

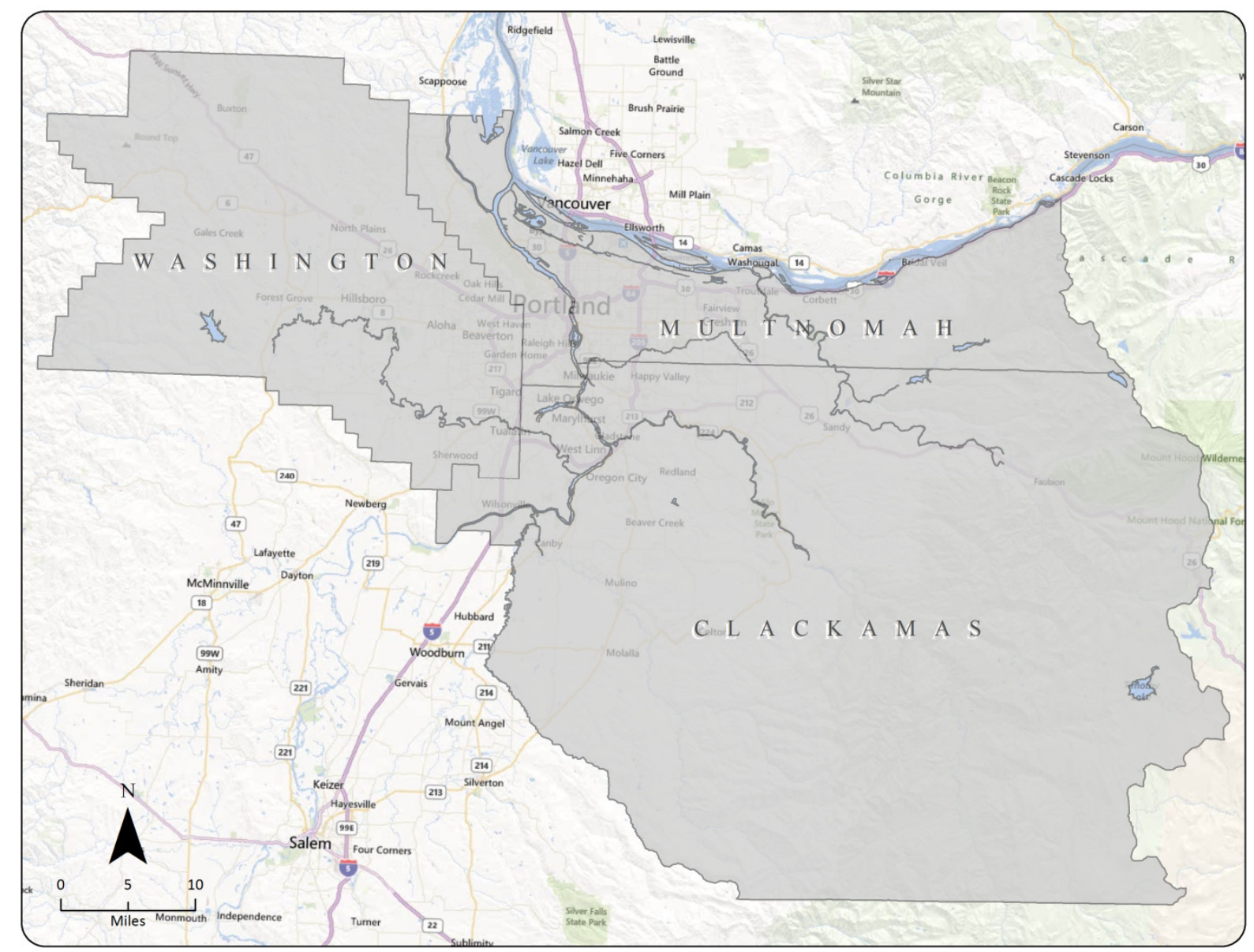

Figure 4-2: Three-county study region of Portland metropolitan region

Data reflecting the built environment, which were used to help quantify the concept of data utility, were supplied by several different resources. Two built environment measures classified as density calculations were explored in this empirical application: population and employment density. Similar to the examination into identity disclosure risk, data used to calculate population density were provided by the 2010 Census. Population density was operationalized at both the block group geography; thus, depending on the selected scenario for donut size, the repositioned household may land within any block group located in the census geography. The variance between the population density of the actual household location and geographically perturbed household location was found as a component of the PRMSE calculation for data utility. A similar approach was used for the other density measure of jobs per acre. Information on this built environment measure was supplied by the Census Bureau's 2010 Longitudinal Employer-Household Dynamics (LEHD) dataset through use of the total number of jobs field within the workplace area characteristic subset. Akin to population density, the measure of employment density was operationalized at the block group level using land area. For the design built environment measure 
of intersection density, the Census Bureau's 2010 Topologically Integrated Geographic Encoding and Referencing (TIGER) dataset was manipulated to count the number of intersections with more than three legs per block group. As with the previous two built environment measures, the potential to use completely disaggregate measures of intersection density exists with improved computational power and data extending across the entire study area.

Two additional built environment variables classified as distance to transit measures were also estimated in this empirical application to assess the utility of the geographically perturbed dataset. The disaggregate measures of miles to nearest bus stop and distance to nearest rail station were calculated by using Metro's 2010 Regional Land Information System (RLIS) dataset (Metro is Portland's regional government). The ability to use a dataset with a higher resolution for these distance measures was due to the fact that all bus stops and rail stations in the three-county region are located within Metro's jurisdiction, which does not extend to the farthest stretches of the chosen study area. The distance to the nearest bus stop was a straight-line distance measure from the household location to the nearest TriMet bus stop. The distance to the nearest rail station was a straight-line distance measure from the household location to the nearest MAX light rail station, Portland Streetcar stop, or Westside Express Service (WES) rail station. The difference between the number of miles from the original household location to the nearest public transit station and the distance from the geographically perturbed household location to the nearest station was used when calculating the PRMSE of each household in each scenario.

An examination into the level of disclosure risk and data utility for these five built environment measures was conducted for 25 different combinations of inner- and outer-ring radii. In this application of the donut masking technique, five different k-anonymity statistics were chosen in defining the size of the exclusion area: 25, 50, 100, 250, and 500. For each of these inner-ring radii, a complementary outer-ring radius of one-quarter mile, one-half mile, one mile, one and onehalf miles, and two miles was chosen to establish the different donut sizes. Having settled on these 25 different scenarios, each household in the study area was then spatially perturbed 50 times to produce a range of values to be used in quantifying the average risk of disclosure and utility of the jittered dataset. A description of the results of this empirical application, which was conducted using PostGIS open source software, is provided in the following section. The reader is referred to Appendix A for the code used in the application of the donut masking technique. 


\subsection{RESULTS}

This empirical application of the donut masking technique to the spatial location of households within the Portland metropolitan region offers an introductory examination into the relationship between disclosure risk and data utility in the context of household travel survey data. The use of a geographic perturbation method such as the donut masking technique on disaggregate travel survey data offers an exciting prospect for researchers who are interested in better understanding the complex nature of the links between non-motorized travel and a household's surrounding built environment. Yet, before such household travel survey data may be widely disseminated for public consumption, there is an explicit responsibility on behalf of individuals with access to these finescaled data sources to ensure a fitting level of noise is introduced to the dataset in order to preserve respondent anonymity. Complementarily, individuals in charge of disseminating these data must ensure the level of noise is not so much that researchers utilizing these data for their intended applications are led toward inaccurate conclusions. The following section explores these tradeoffs between disclosure risk and data utility as well as the important concept of data retention in an empirical application of the donut masking technique to households in the Portland metropolitan region.

\subsection{DATA RETENTION ANALYSIS}

In the framework of this data masking technique application, data retention refers to the number of households that are able to be geographically perturbed in a specific scenario. This opening set of analyses is precautious, but mandatory, since the strategy for defining the inner and outer rings of the donut area are not directly associated with one another. The decision to set the inner-ring radius to a distance equivalent to a k-anonymity statistic defined by the data custodian is not related to the selection of a straight-line areal buffer based on common practice for the donut's outer ring. Accordingly, one may imagine a scenario in which a data custodian, who has not given careful consideration to the inherent tradeoff between these expressions of disclosure risk and data utility, may select a high estimated k-anonymity statistic to define the exclusion area and a constrained outer-ring radius to define the boundary of maximum displacement. If the areal buffer defining the outer-ring radius is shorter than the inner-ring radius associated with a higher level of anonymity, then the result is a collapsed donut in which the actual household location has no geographical space to be randomly placed within. The prevalence of the collapsed donut situation in a specific scenario must be understood by the data custodian since the households impacted by these instances must be spatially perturbed within some other scenario or by another technique. Table 5-1 shows the number of households within each of the 25 scenarios that were retained in this geographic perturbation application, while Table 5-2 provides the percentage of retained records out of the sample of 4,824 households.

Table 5-1: Total number of households retained within each scenario

\begin{tabular}{l|l|l|l|l|l}
\hline \multirow{2}{*}{$\begin{array}{l}\text { Estimated } \\
\text { K-Anonymity }\end{array}$} & \multicolumn{5}{|l}{ Outer-Ring Radius in Miles } \\
\cline { 2 - 6 } & 0.25 & 0.50 & 1.00 & 1.50 & 2.00 \\
\hline 25 & 4,453 & 4,650 & 4,789 & 4,823 & 4,823 \\
\hline 50 & 4,309 & 4,570 & 4,772 & 4,807 & 4,814 \\
\hline 100 & 4,142 & 4,467 & 4,650 & 4,772 & 4,789 \\
\hline 250 & 3,449 & 4,286 & 4,545 & 4,637 & 4,743 \\
\hline
\end{tabular}




\begin{tabular}{|c|c|c|c|c|c|}
\hline 500 & 1,484 & 4,010 & 4,403 & 4,557 & 4,637 \\
\hline \multirow{2}{*}{$\begin{array}{l}\text { Estimated } \\
\text { K-Anonymity }\end{array}$} & \multicolumn{5}{|c|}{ Outer-Ring Radius in Miles } \\
\hline & 0.25 & 0.50 & 1.00 & 1.50 & 2.00 \\
\hline 25 & $92.3 \%$ & $96.4 \%$ & $99.3 \%$ & $100.0 \%$ & $100.0 \%$ \\
\hline 50 & $89.3 \%$ & $94.7 \%$ & $98.9 \%$ & $99.6 \%$ & $99.8 \%$ \\
\hline 100 & $85.9 \%$ & $92.6 \%$ & $96.4 \%$ & $98.9 \%$ & $99.3 \%$ \\
\hline 250 & $71.5 \%$ & $88.8 \%$ & $94.2 \%$ & $96.1 \%$ & $98.3 \%$ \\
\hline 500 & $30.8 \%$ & $83.1 \%$ & $91.3 \%$ & $94.5 \%$ & $96.1 \%$ \\
\hline
\end{tabular}

A review of the above tables confirms the indirect link between the two radii that comprise the choice of donut size described in the previous paragraph. Intuitively, an increase in the straightline distance of the outer-ring radius within a specified level of k-anonymity resulted in either an increase or preservation in the number of households retained in the spatially perturbed datasets. A consistent and negative trend was found as the estimated k-anonymity statistic defining the inner-ring radius was increased within a specified outer-ring radius. Also of note, even though the two scenarios with an estimated k-anonymity statistic of 25 and an outer-ring radius of at least one and one-half miles appeared to retain 100 percent of the sampled observations in the altered dataset, there was one record in each of these scenarios with a collapsed donut. As such, the data custodian must accept either a lower level of data utility or a higher level of disclosure risk when geographically perturbing this household location with this version of the donut masking technique.

\subsubsection{Data Retention within Subcategories of Population Density}

While an examination into the number and percent of households retained within each scenario of the geographic perturbation process begins to highlight the sort of tradeoffs that occur when the data custodian balances disclosure risk and data utility, this aggregate analysis only scratches the surface of how these themes interact with data retention. Understanding that the inner-ring radius is directly dependent on the residential density across the survey respondent's census tract, the data custodian may begin to see how a household within a more rural context that is provided a high level of anonymity with a small outer-ring radius is more likely to have a collapsed donut than a household in an urban context within the same scenario. Accordingly, the data custodian may wish to examine how data retention varies across different intensities of built environment measures within the same scenario, especially population density.

In order to further inspect data retention trends for the altered datasets across the scenarios, the original households were allocated to quintiles based on the population density of the tracts in the study area. An equal interval classification scheme of the tracts was favored over a natural breaks scheme for this built environment measure since population density can theoretically increase without any increase on the same space of land due to intensification in household size or vertical growth in residential complexes. While employment density may also be categorized with this approach, a natural classification scheme would be more suitable for intersection density or distance to nearest transit station since thinking of intensification in these built environment measures does not favor the same logic. The distribution of tracts divided into quintiles based on population density for the study area is displayed in Figure 5-1. For the quantile classification of 
population density within the Portland area, 832 households were sampled in the travel survey located in a tract classified in the first grouping; 960 households were in the second class; 1,079 households were in the third class; 982 households were in the fourth class; and 971 households were located in those census tracts with the highest population density.

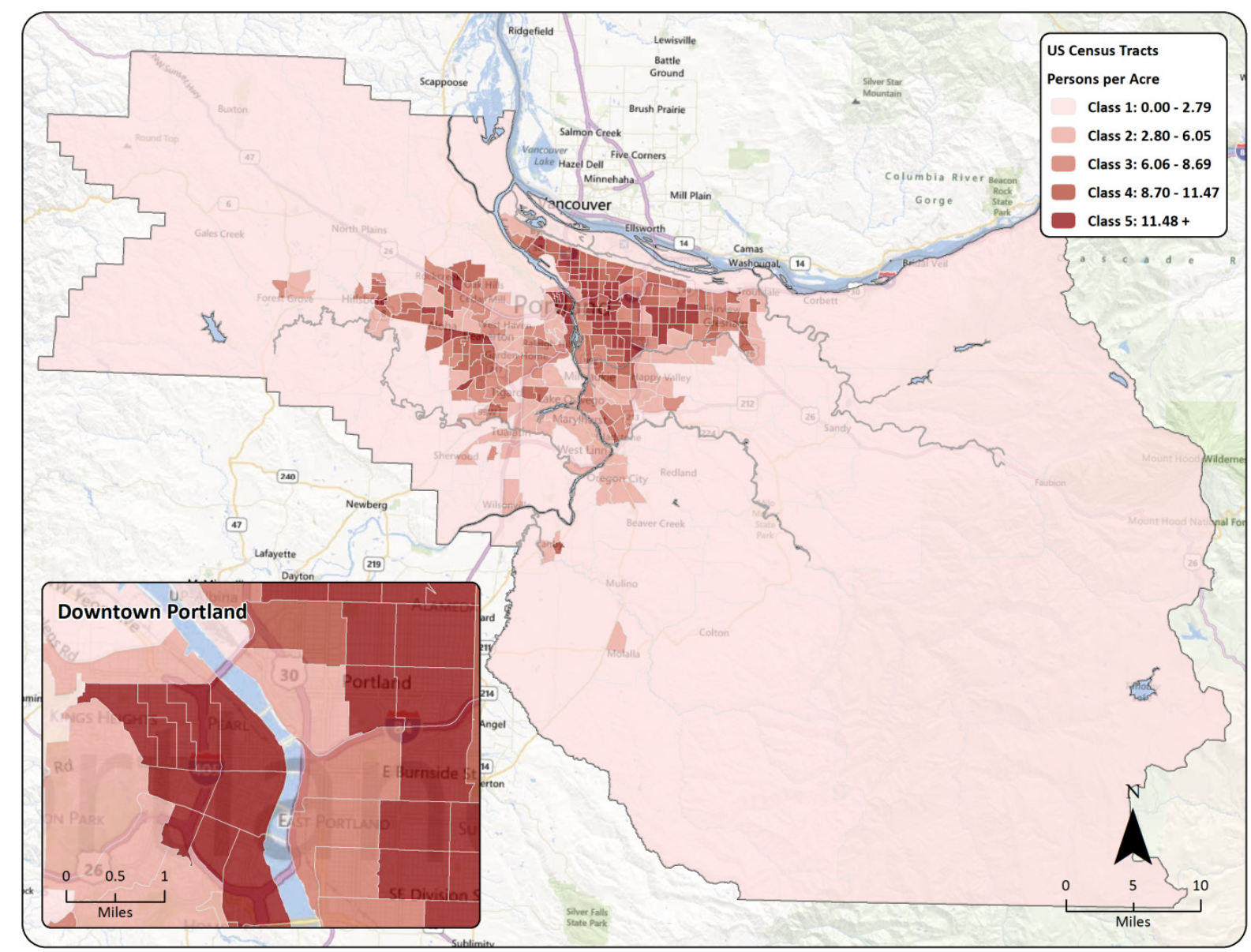

Figure 5-1: Quintile classification of population density for U.S. Census tracts in Portland metropolitan region

Having defined the classified census tracts across the study area into different contexts based on population density, the data custodian may now more closely assess trends in data retention for the 25 scenarios across five different spatial contexts. Tables 5-3 through 5-7 outline the total number and percent of retained households within each scenario, beginning with the first quintile and proceeding through the fifth quintile and reflecting the 20 percent of census tracts in the study area with the highest population density.

Table 5-3: Number and percent of retained households per scenario within class 1 for population density

\begin{tabular}{|c|c|c|c|c|c|c|c|c|c|c|}
\hline \multirow{3}{*}{$\begin{array}{l}\text { Estimated } \\
\text { K- } \\
\text { Anonymity }\end{array}$} & \multicolumn{10}{|c|}{ Outer-Ring Radius in Miles } \\
\hline & \multicolumn{2}{|l|}{0.25} & \multicolumn{2}{|l|}{0.50} & \multicolumn{2}{|l|}{1.00} & \multicolumn{2}{|l|}{1.50} & \multicolumn{2}{|l|}{2.00} \\
\hline & Count & Percent & Count & Percent & Count & Percent & Count & Percent & Count & Percent \\
\hline 25 & 461 & $55 \%$ & 658 & $79 \%$ & 797 & $96 \%$ & 831 & $99 \%$ & 831 & $99 \%$ \\
\hline 50 & 317 & $38 \%$ & 578 & $70 \%$ & 780 & $94 \%$ & 815 & $98 \%$ & 822 & $99 \%$ \\
\hline 100 & 150 & $18 \%$ & 475 & $57 \%$ & 658 & $79 \%$ & 780 & $94 \%$ & 797 & $96 \%$ \\
\hline 250 & 0 & $0 \%$ & 294 & $35 \%$ & 553 & $67 \%$ & 645 & $78 \%$ & 751 & $90 \%$ \\
\hline
\end{tabular}




\begin{tabular}{l|l|l|l|l|l|l|l|l|l|l}
\hline 500 & 0 & $0 \%$ & 30 & $4 \%$ & 411 & $49 \%$ & 565 & $68 \%$ & 645 & $78 \%$ \\
\hline
\end{tabular}

Table 5-4: Number and percent of retained households per scenario within class 2 for population density

\begin{tabular}{|c|c|c|c|c|c|c|c|c|c|c|}
\hline \multirow{3}{*}{$\begin{array}{l}\text { Estimated } \\
\text { K- } \\
\text { Anonymity }\end{array}$} & \multicolumn{10}{|c|}{ Outer-Ring Radius in Miles } \\
\hline & \multicolumn{2}{|l|}{0.25} & \multicolumn{2}{|l|}{0.50} & \multicolumn{2}{|l|}{1.00} & \multicolumn{2}{|l|}{1.50} & \multicolumn{2}{|l|}{2.00} \\
\hline & Count & Percent & Count & Percent & Count & Percent & Count & Percent & Count & Percent \\
\hline 25 & 960 & $100 \%$ & 960 & $100 \%$ & 960 & $100 \%$ & 960 & $100 \%$ & 960 & $100 \%$ \\
\hline 50 & 960 & $100 \%$ & 960 & $100 \%$ & 960 & $100 \%$ & 960 & $100 \%$ & 960 & $100 \%$ \\
\hline 100 & 960 & $100 \%$ & 960 & $100 \%$ & 960 & $100 \%$ & 960 & $100 \%$ & 960 & $100 \%$ \\
\hline 250 & 417 & $43 \%$ & 960 & $100 \%$ & 960 & $100 \%$ & 960 & $100 \%$ & 960 & $100 \%$ \\
\hline 500 & 0 & $0 \%$ & 948 & $99 \%$ & 960 & $100 \%$ & 960 & $100 \%$ & 960 & $100 \%$ \\
\hline
\end{tabular}

Table 5-5: Number and percent of retained households per scenario within class 3 for population density

\begin{tabular}{|c|c|c|c|c|c|c|c|c|c|c|}
\hline \multirow{3}{*}{$\begin{array}{l}\text { Estimated } \\
\text { K- } \\
\text { Anonymity }\end{array}$} & \multicolumn{10}{|c|}{ Outer-Ring Radius in Miles } \\
\hline & \multicolumn{2}{|l|}{0.25} & \multicolumn{2}{|l|}{0.50} & \multicolumn{2}{|l|}{1.00} & \multicolumn{2}{|l|}{1.50} & \multicolumn{2}{|l|}{2.00} \\
\hline & Count & Percent & Count & Percent & Count & Percent & Count & Percent & Count & Percent \\
\hline 25 & 1,079 & $100 \%$ & 1,079 & $100 \%$ & 1,079 & $100 \%$ & 1,079 & $100 \%$ & 1,079 & $100 \%$ \\
\hline 50 & 1,079 & $100 \%$ & 1,079 & $100 \%$ & 1,079 & $100 \%$ & 1,079 & $100 \%$ & 1,079 & $100 \%$ \\
\hline 100 & 1,079 & $100 \%$ & 1,079 & $100 \%$ & 1,079 & $100 \%$ & 1,079 & $100 \%$ & 1,079 & $100 \%$ \\
\hline 250 & 1,079 & $100 \%$ & 1,079 & $100 \%$ & 1,079 & $100 \%$ & 1,079 & $100 \%$ & 1,079 & $100 \%$ \\
\hline 500 & 52 & $5 \%$ & 1,079 & $100 \%$ & 1,079 & $100 \%$ & 1,079 & $100 \%$ & 1,079 & $100 \%$ \\
\hline
\end{tabular}

Table 5-6: Number and percent of retained households per scenario within class 4 for population density

\begin{tabular}{|c|c|c|c|c|c|c|c|c|c|c|}
\hline \multirow{3}{*}{$\begin{array}{l}\text { Estimated } \\
\text { K- } \\
\text { Anonymity }\end{array}$} & \multicolumn{10}{|c|}{ Outer-Ring Radius in Miles } \\
\hline & \multicolumn{2}{|l|}{0.25} & \multicolumn{2}{|l|}{0.50} & \multicolumn{2}{|l|}{1.00} & \multicolumn{2}{|l|}{1.50} & \multicolumn{2}{|l|}{2.00} \\
\hline & Count & Percent & Count & Percent & Count & Percent & Count & Percent & Count & Percent \\
\hline 25 & 982 & $100 \%$ & 982 & $100 \%$ & 982 & $100 \%$ & 982 & $100 \%$ & 982 & $100 \%$ \\
\hline 50 & 982 & $100 \%$ & 982 & $100 \%$ & 982 & $100 \%$ & 982 & $100 \%$ & 982 & $100 \%$ \\
\hline 100 & 982 & $100 \%$ & 982 & $100 \%$ & 982 & $100 \%$ & 982 & $100 \%$ & 982 & $100 \%$ \\
\hline 250 & 982 & $100 \%$ & 982 & $100 \%$ & 982 & $100 \%$ & 982 & $100 \%$ & 982 & $100 \%$ \\
\hline 500 & 470 & $48 \%$ & 982 & $100 \%$ & 982 & $100 \%$ & 982 & $100 \%$ & 982 & $100 \%$ \\
\hline
\end{tabular}

Table 5-7: Number and percent of retained households per scenario within class 5 for population density

\begin{tabular}{|c|c|c|c|c|c|c|c|c|c|c|}
\hline \multirow{3}{*}{$\begin{array}{l}\text { Estimated } \\
\text { K- } \\
\text { Anonymity }\end{array}$} & \multicolumn{10}{|c|}{ Outer-Ring Radius in Miles } \\
\hline & \multicolumn{2}{|l|}{0.25} & \multicolumn{2}{|l|}{0.50} & \multicolumn{2}{|l|}{1.00} & \multicolumn{2}{|l|}{1.50} & \multicolumn{2}{|l|}{2.00} \\
\hline & Count & Percent & Count & Percent & Count & Percent & Count & Percent & Count & Percent \\
\hline 25 & 971 & $100 \%$ & 971 & $100 \%$ & 971 & $100 \%$ & 971 & $100 \%$ & 971 & $100 \%$ \\
\hline 50 & 971 & $100 \%$ & 971 & $100 \%$ & 971 & $100 \%$ & 971 & $100 \%$ & 971 & $100 \%$ \\
\hline 100 & 971 & $100 \%$ & 971 & $100 \%$ & 971 & $100 \%$ & 971 & $100 \%$ & 971 & $100 \%$ \\
\hline 250 & 971 & $100 \%$ & 971 & $100 \%$ & 971 & $100 \%$ & 971 & $100 \%$ & 971 & $100 \%$ \\
\hline 500 & 962 & $99 \%$ & 971 & $100 \%$ & 971 & $100 \%$ & 971 & $100 \%$ & 971 & $100 \%$ \\
\hline
\end{tabular}

In total, a glance across the above tables sheds further light on a handful of trends concerning the retention of records in an altered dataset, and the application of the donut masking technique with intentions for minimum disclosure risk and maximum data utility. An instinctive finding from this further parsing of the records was a confirmation of the lone observation in the sample not being jittered because of a collapsed donut related to its rural context. The absence of the household from this application is revealed in Table 5-3, where 99.9 percent of the households were retained in the scenario with the lowest specified k-anonymity statistic and greatest outer-ring radius. By shifting 
the balance from a scenario maximizing disclosure risk and minimizing data utility to the preferred scenario minimizing disclosure risk and maximizing data utility, the results in Table 5-4 exhibit the frequency of households in a rural context being susceptible to a collapsed donut in this application of the perturbation technique. Households residing in a tract designated in the lowest quintile for population density were not able to have their locations spatially perturbed when the outer-ring radius was 0.25 miles and the inner-ring radius was defined by a k-anonymity statistic of either 250 or 500. Table 5-4 shows that those households located within tracts categorized in the second quintile were also not geographically perturbed when the outer-ring radius was 0.25 miles and the k-anonymity was set at 500. A glance at Table 5-5 shows the percent of retained households in this preferred scenario to only slightly increase when located in a tract in the third quintile to 4.8 percent; while, retained households remain under 50 percent for this scenario when the household resides in a tract marked by the second highest categorization population density (Table 5-6). Conditional upon the value of an observation in the survey sample, the data custodian may only consider using the scenario with the highest anonymity and potential data utility for those households located in the most urban of contexts; however, Table 5-7 shows nine records in this particular exploration would still not be retained.

While this analysis of data retention investigated the equal breaks of census tracts in regard to population density because of its connection to the size of the exclusion area, a researcher may be more interested in other built environment measures. For instance, a data researcher may be interested in the association between non-motorized mode choices for work-related purposes and employment density. In such applications, the data custodian should consider how levels of data retention are distributed across different classifications of the built environment to ensure the researcher is provided ample records to conduct the appropriate analysis. Clearly, data retention is an essential aspect to consider when releasing disaggregate household travel surveys. However, the theme of disclosure risk will likely carry more weight in the decision of what perturbation process is most suitable for publicly releasing a disaggregate dataset.

\subsection{DISCLOSURE RISK ANALYSIS}

To supplement the above examination into data retention, an analysis into the vulnerability of a sampled household to identity disclosure was conducted for each of the 25 scenarios. Adhering to the aforementioned hierarchical framework for disclosure risk and data utility, the following evaluation assesses the potential of a first-tier risk in which the data intruder is able to make a oneto-one correspondence without the use of external data, local knowledge or analytical effort. As such, this disclosure risk analysis is strictly concerned with the level of anonymity introduced to a geographically perturbed dataset created by the use of this version of the donut masking technique. Moreover, the risk of a household having their identity disclosed is measured through the estimation of several k-anonymity statistics derived from 50 geographic perturbations of the original household location across 25 combinations of inner- and outer-ring radii. While the minimum k-anonymity statistic per scenario is related to the distance of the inner-ring radius and the maximum $\mathrm{k}$ - anonymity statistic is connected to the outer-ring radius, a proper understanding of the mean and median values is less clear and ultimately dependent on the discrepancy between distances of the chosen radii. The following analysis will identify trends that arose through a general examination of disclosure risk across these scenarios in addition to a detailed assessment of anonymity in relation to the grouping of sampled households by the intensity of select built environment measures surrounding their location. 
Table 5-8 shows the average minimum and maximum k-anonymity statistics resulting from 50 different geographic perturbations of each household surveyed in the three-county study area across the 25 different combinations of inner- and outer-ring radii. In those scenarios seeking to maximize data utility, the value of the average minimum k-anonymity statistic nearly mirrors the absolute minimum value for the statistic, which was specified by the data custodian and used to limit the level of identity disclosure. Predictably, as the size of the outside areal buffer increases, the gap between the average minimum k-anonymity statistic and value describing the absolute minimum k-anonymity statistic for each scenario widens. The greatest difference, 54 households, was found to exist when the absolute minimum k-anonymity statistic was 500 and the household had the potential to be repositioned two miles from its actual location. Similarly, the value of the average maximum k-anonymity statistic for all scenarios shown in Table 5-9 was unsurprisingly the greatest in this scenario seeking to minimize disclosure risk at the tradeoff of potentially low data utility.

Table 5-8: Average minimum and maximum k-anonymity statistic from 50 simulations per scenario

\begin{tabular}{|c|c|c|c|c|c|c|c|c|c|c|}
\hline \multirow{3}{*}{$\begin{array}{l}\text { Estimated } \\
\text { K- } \\
\text { Anonymity }\end{array}$} & \multicolumn{10}{|c|}{ Outer-Ring Radius in Miles } \\
\hline & \multicolumn{2}{|l|}{0.25} & \multicolumn{2}{|c|}{0.50} & \multicolumn{2}{|l|}{1.00} & \multicolumn{2}{|l|}{1.50} & \multicolumn{2}{|l|}{2.00} \\
\hline & Min. & Max. & Min. & Max. & Min. & Max. & Min. & Max. & Min. & Max \\
\hline 25 & 33 & 451 & 45 & 1,680 & 63 & 4,860 & 68 & 6,689 & 72 & 7,499 \\
\hline 50 & 58 & 466 & 71 & 1,705 & 89 & 4,868 & 94 & 6,702 & 97 & 7,514 \\
\hline 100 & 107 & 483 & 120 & 1,753 & 140 & 5,007 & 147 & 6,764 & 147 & 7,588 \\
\hline 250 & 254 & 548 & 270 & 1,826 & 293 & 5,137 & 299 & 7,016 & 302 & 7,715 \\
\hline 500 & 502 & 789 & 518 & 1,937 & 544 & 5,329 & 551 & 7,169 & 554 & 7,953 \\
\hline
\end{tabular}

Table 5-9: Average median and mean k-anonymity statistic from 50 simulations per scenario

\begin{tabular}{|c|c|c|c|c|c|c|c|c|c|c|}
\hline \multirow{3}{*}{$\begin{array}{l}\text { Estimated } \\
\text { K- } \\
\text { Anonymity }\end{array}$} & \multicolumn{10}{|c|}{ Outer-Ring Radius in Miles } \\
\hline & \multicolumn{2}{|l|}{0.25} & \multicolumn{2}{|l|}{0.50} & \multicolumn{2}{|l|}{1.00} & \multicolumn{2}{|l|}{1.50} & \multicolumn{2}{|l|}{2.00} \\
\hline & Median & Mean & Median & Mean & Median & Mean & Median & Mean & Median & Mean \\
\hline 25 & 220 & 227 & 668 & 728 & 1,445 & 1,738 & 1,805 & 2,237 & 1,954 & 2,546 \\
\hline 50 & 242 & 249 & 695 & 757 & 1,476 & 1,766 & 1,829 & 2,263 & 1,988 & 2,482 \\
\hline 100 & 277 & 283 & 747 & 807 & 1,562 & 1,855 & 1,907 & 2,331 & 2,074 & 2,559 \\
\hline 250 & 390 & 394 & 880 & 933 & 1,740 & 2,022 & 2,116 & 2,539 & 2,254 & 2,730 \\
\hline 500 & 633 & 638 & 1,082 & 1,130 & 2,027 & 2,282 & 2,413 & 2,804 & 2,564 & 3,016 \\
\hline
\end{tabular}

Turning to an evaluation of the average median and mean k-anonymity statistic for the different applications of the donut masking technique, the familiar trend of an increase in each measure continued in circumstances where either radius was increased and the alternative radius remained unchanged. The lowest value for each averaged measure of central tendency was the lowest for the scenario with the highest risk of disclosure and lowest potential for spatial error introduction; whereas, the scenario with the lowest risk of identity disclosure and highest potential for spatial error produced the highest average median and mean k-anonymity statistic of all the scenarios. As both of these trends were anticipated, the data custodian may wish to revisit the thought of how best to balance these two themes since the researcher will be most interested in maximizing the utility of the altered dataset. Accordingly, the data custodian should examine these summary statistics for circumstances in which the level of anonymity appears to taper when iteratively extending the outer-ring radius. Figure 5-2 provides a bubble chart of the average median kanonymity statistic across the 25 scenarios that may help the data custodian visualize any tipping 
point in the tradeoff between disclosure risk and data utility. A parallel illustration of the average mean k-anonymity statistic has not been provided since its portrayal did not offer any additional insight beyond those trends in the average median k-anonymity statistic described below.

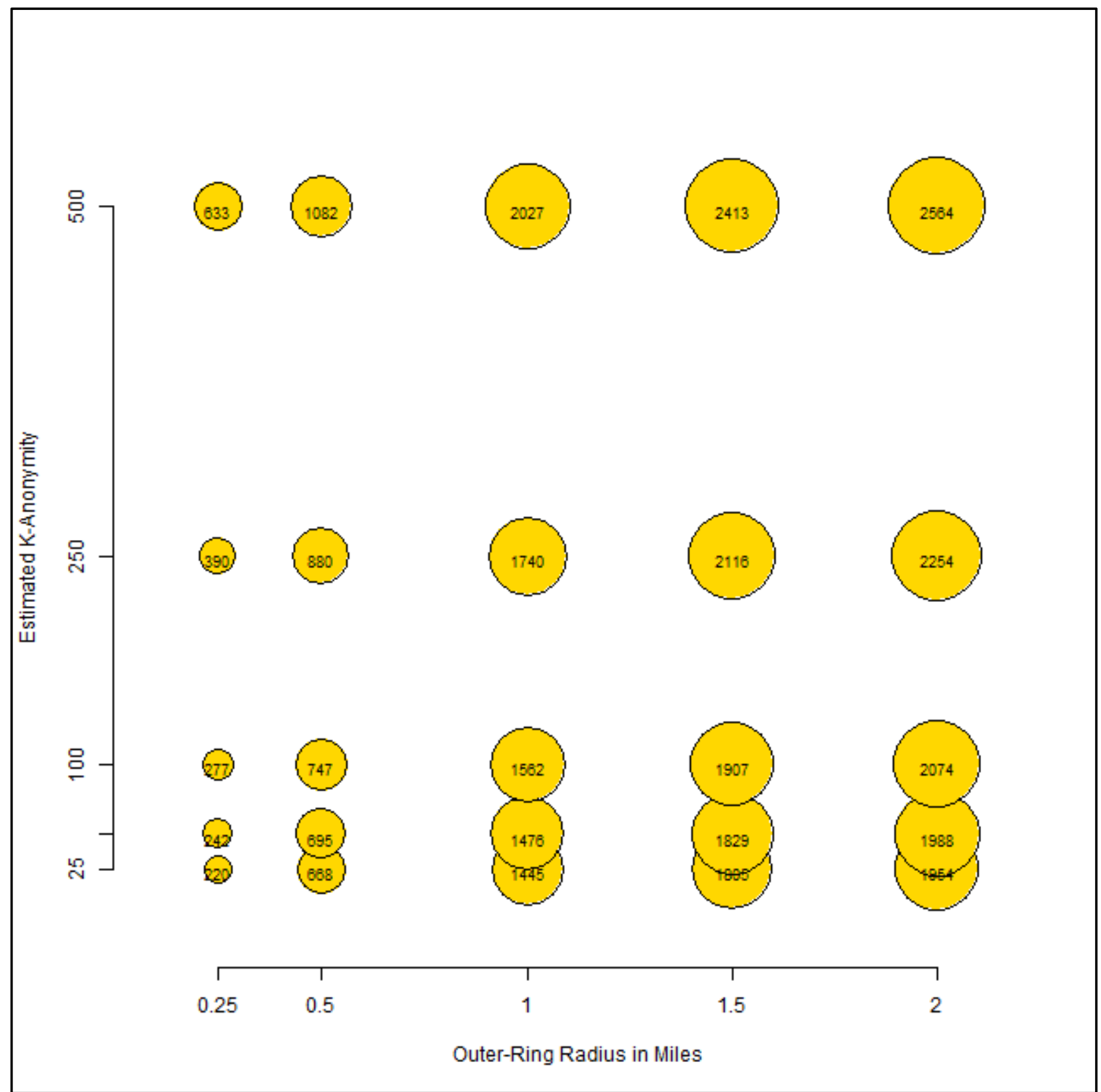

Figure 5-2: Level of anonymity per scenario over 50 simulations (average median k-anonymity)

A visual inspection of Figure 5-2, which provides bubbles sized relatively to the average median k-anonymity statistic for all 25 scenarios, unveils trends regarding the conciliation in potential data utility that may be pursued by the data custodian for relatively small increases in respondent anonymity. Chiefly, for all anonymity levels analyzed in this empirical application, the extension of the outer-ring radius appears to greatly improve the overall average anonymity of participants for the smaller outer-ring sizes with this improvement diminishing in magnitude for scenarios with an outer-ring radius of at least one mile. On the other hand, the value of the average median k- 
anonymity statistic appears to steadily increase at a slower rate as the outer-ring radius is kept at a specified distance and the absolute minimum level of anonymity is increased. Therefore, depending on the requisite anonymity level sought by the data custodian and value placed on data retention, the data custodian may choose to limit the displacement distance to somewhere between 1.0-1.5 miles and increase the k-anonymity level to a tolerable threshold. However, this suggestion is based on the decision of the data custodian to mask the entire dataset with the same specification of inner- and outer-ring radius. The following analyses look at how disclosure risk within each scenario is impacted by the grouping of the sampled households' built environment into different classes.

\subsubsection{Disclosure Risk within Subcategories of Density Measures}

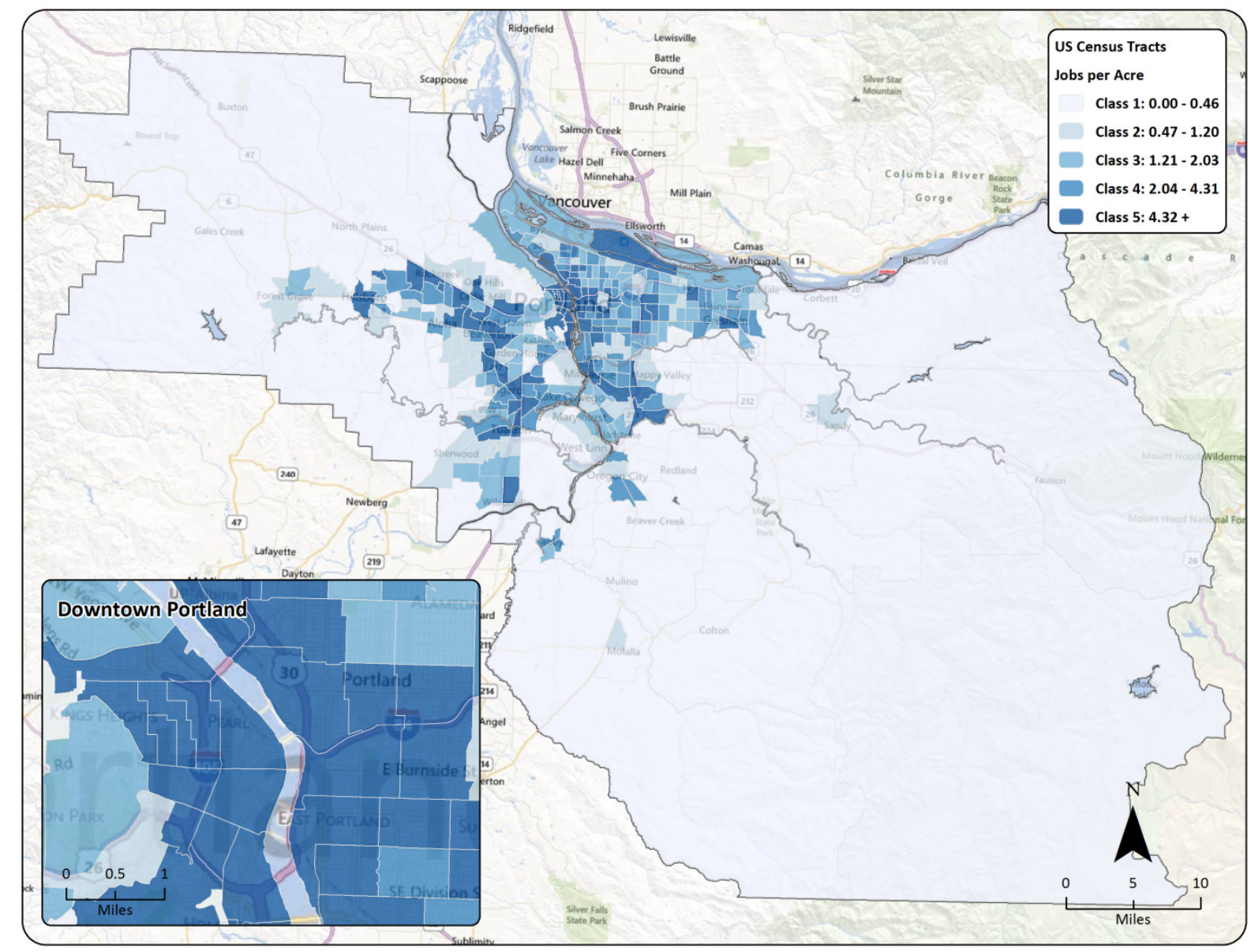

Figure 5-3: Quintile classification of employment density for U.S. Census tracts in Portland metropolitan region

As with the analysis pertaining to data retention, a data custodian may wish to examine whether trends found on the aggregate hold when the 25 scenarios are further divided into classifications based on certain levels of intensity in the built environment surrounding a sampled household. To advance this understanding of disclosure risk in terms of inner- and outer-ring specification, the census tracts of the three-county study area were divided into five equal categories based on the density measures of persons per acre and jobs per acre. Once the study area was fragmented into 
these quintiles, the actual location of each sampled household was assigned to one of the five classes of the respective built environment measure. Figure 5-1 earlier introduced the spatial layout of these quintiles in regard to population density, while Figure 5-3 offers a map of the distribution of census tracts defined by the five employment density categories. As with the map classifying tracts based on population density, the map describing employment density across the Portland region also denotes the values that define membership to each of the quintiles.

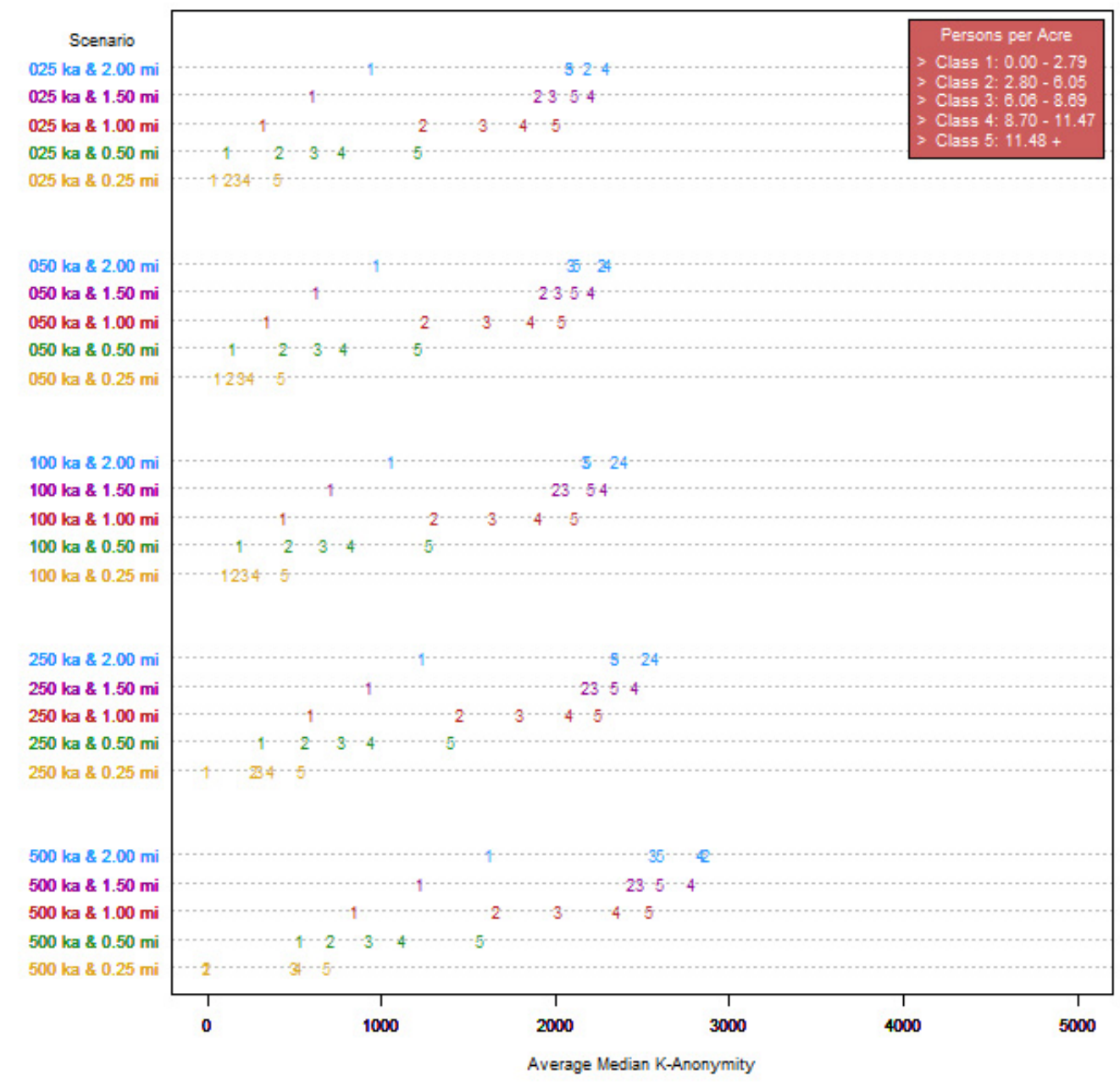

Figure 5-4: Level of anonymity per scenario over 50 simulations (quintiles for population density)

Figure 5-4 is a dot chart plotting the average median k-anonymity statistic for each quintile of population density in the 25 different scenarios. This representation of anonymity was estimated by finding the median estimated k-anonymity statistic calculated for 50 geographic perturbations of each observation in the original dataset and then taking the average of these median values for all households located in a tract classified by a particular quintile. Overall, the average anonymity continues to increase when the distance of either radius is held constant and the radius of the alternative ring is extended. These trends are somewhat intuitive and were noted in the previous aggregate analysis; yet, more interesting findings are revealed by examining how average anonymity levels change within different contexts of population density. A glance at anonymity levels for households located in a rural context noted by the first quintile shows a fairly constant 
rate of growth when the k-anonymity statistic defining the inner ring is held constant and the outer ring is allowed to expand. However, the pattern changes for those households located in tracts with a greater population density. The average median k-anonymity values within each group of innerring radii increases at a nonlinear rate, but slows considerably when the outer-ring radius is a distance of at least 1.5 miles for households in the most urban context. Moreover, the levels of anonymity for those households located outside of the tracts defined by the first class tend to converge when the outer ring is set to the two-mile extent. This finding may potentially be due to the constraint in this application to define the outer-ring buffer as the tract boundary when the displacement distance could potentially place the spatially perturbed household outside of its original census tract. In order to see how this constraint may be impacting this analysis of disclosure risk, the grouping of the study area by the complementary measure of employment density was explored. Figure 5-5 represents a dot chart similar to Figure 5-4, which categorizes the built environments of surveyed households by equal intervals of employment density.

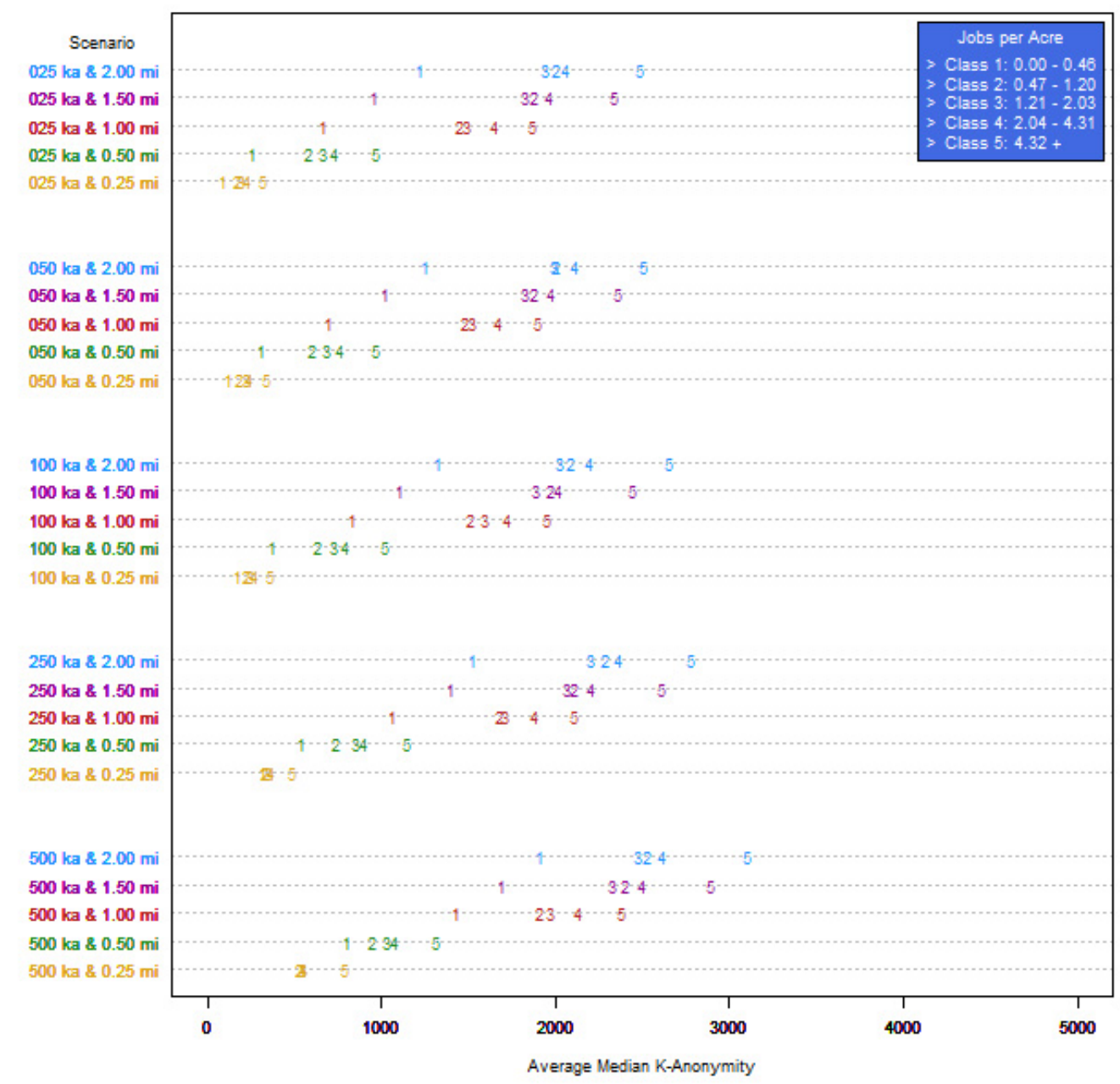

Figure 5-5: Level of anonymity per scenario over 50 simulations (quintiles for employment density)

By examining the average level of anonymity for scenarios divided into five employment density classes describing the census tract of a survey respondent's residence, many of the same trends 
noted in the analysis of population density continue to hold. Additionally, the same clustering of average median k-anonymity statistic values for the four densest quintiles does not appear when looking at the employment density of a census tract; instead, the bunching occurs for households located in the middle three quintiles. Of note, the values of the average level of anonymity for the quintiles of the five scenarios with an outer ring of one mile in both Figures 5-4 and 5-5 are sequential in order. This observed behavior is desirable, especially when examining population density, and leads further credence to the notion that the data custodian using this application of the donut masking technique may wish to confine any displacement of the household's location by this distance.

\subsubsection{Disclosure Risk within Subcategories of Design Measures}

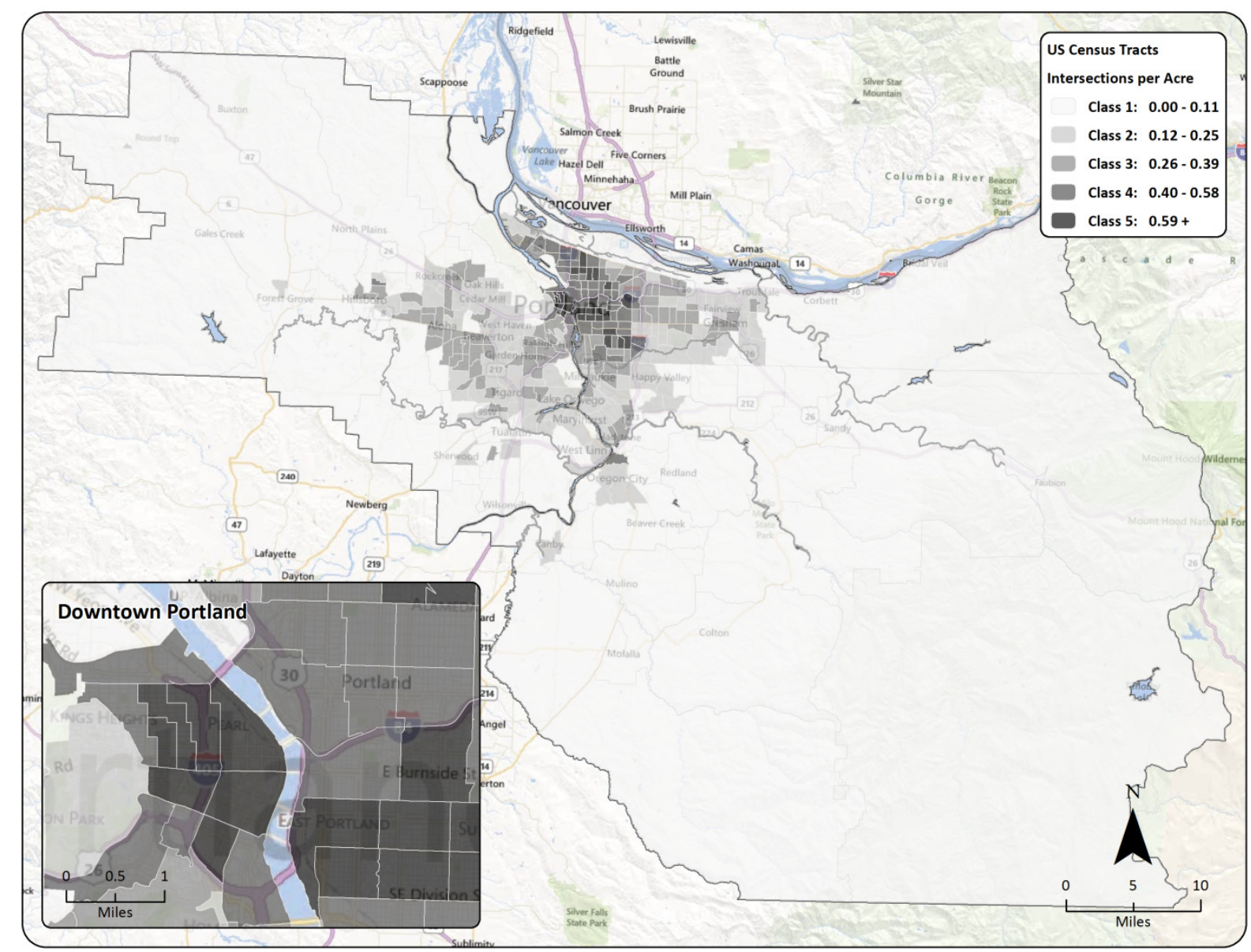

Figure 5-6: Natural break classification of intersection density for U.S. Census tracts in Portland metropolitan region

Building on the previous analyses investigating the degree of disclosure risk characteristic of different divisions of the study area by density measures, the following subsection examines the average level of anonymity for the various scenarios when categorizing the household's built environment by intersection density. The number of intersections per acre, unlike population or employment density, was discretized by using a natural breaks classification scheme since this design measure cannot increase on the same space of land. Intersections cannot be placed on top of one another like households or jobs can through vertical growth. Figure 5-6 is a map of census 
tracts in the study area divided into five natural breaks, with the natural break intervals being noted in the legend of the figure. In addition to this map outlining the spatial distribution of the different levels of intersection density across the region is Figure 5-7, which is a plot of the average median k-anonymity statistic for each natural break class of intersection density within the 25 different scenarios.

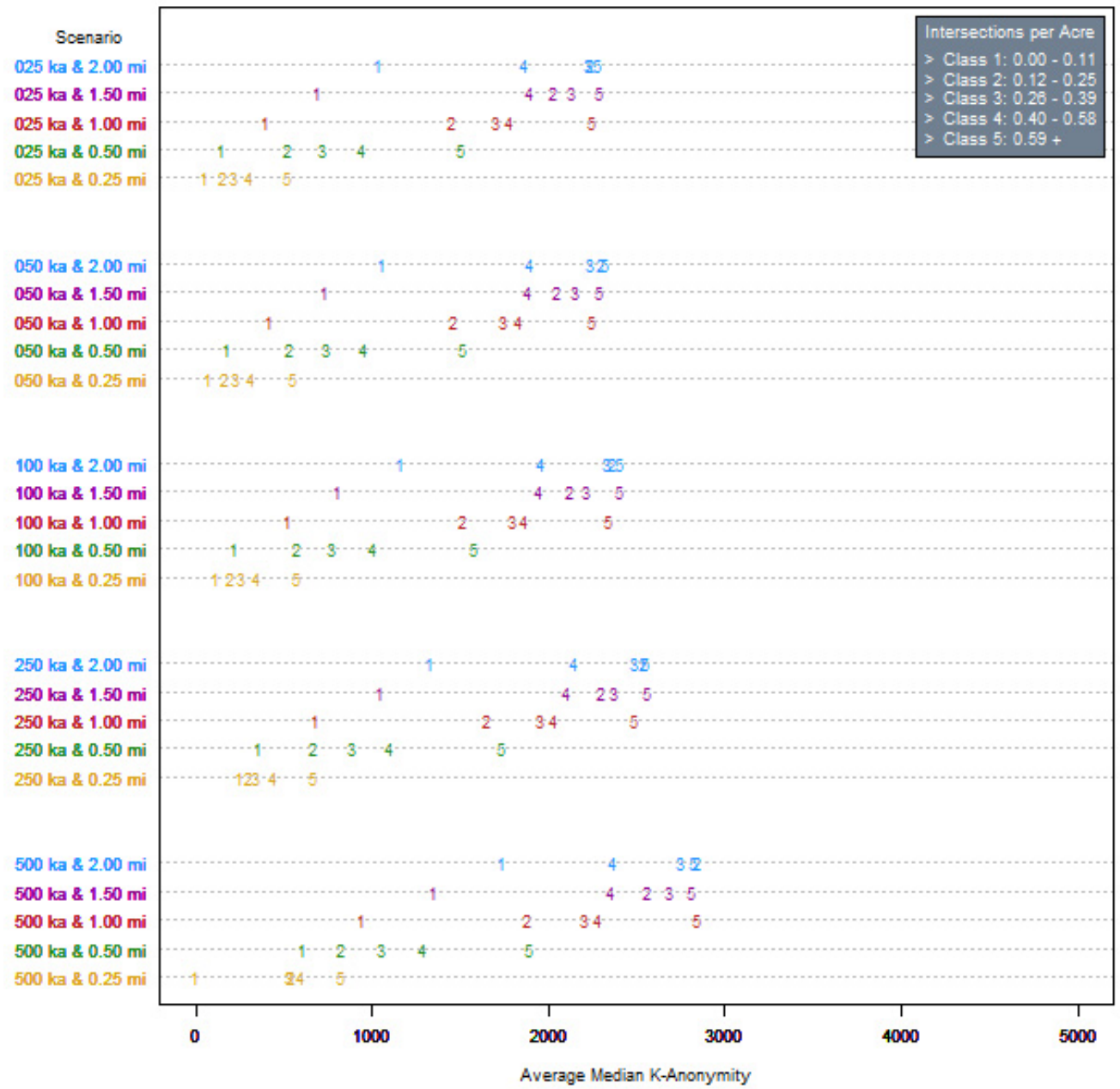

Figure 5-7: Level of anonymity per scenario over 50 simulations (natural breaks for intersection density)

Many of the trends described in the analyses of different population and employment density contexts are also evident in the above illustration of intersection density. Overall, a look at the average median anonymity level for scenarios of a comparable outer-ring radius showed that an increase in the size of the exclusion area for a scenario led to a heightened level of anonymity. The same tendency was evident for the average anonymity of scenarios grouped by an identical anonymity constraint for their exclusion area as the outer ring was extended. The lone exception to this trend occurring for the scenarios in which the donut masking technique was applied to a household was located in those census tracts with the greatest intersection density. Also, the order of the natural break classifications of census tracts is sequential for each of those scenarios in which the donut masking technique specified an outer-ring radius of one mile or less. This 
occurrence was also found in the analysis of previous built environment measures with either a one-half or one mile outer-ring radius.

\subsubsection{Disclosure Risk within Subcategories of Distance to Transit Measures}

While the classification of the previous three built environment measures differed in scheme, the trends in the level of disclosure risk found across these varying gradations of a household's built environment were comparable. Such an association is simply the product of each analysis being an in-depth extension of the more aggregate analysis described in the beginning of this section. However, each of these three extensions into disclosure risk of the 25 scenarios were completed by using classifications of the built environment based on area measures, which may have led to certain inconsistencies related to how the study area was segmented. To bolster this evaluation of disclosure risk, the following subsection provides an assessment of average anonymity levels for households in the study region whose locations are categorized based on their accessibility to the nearest bus stop and rail station.

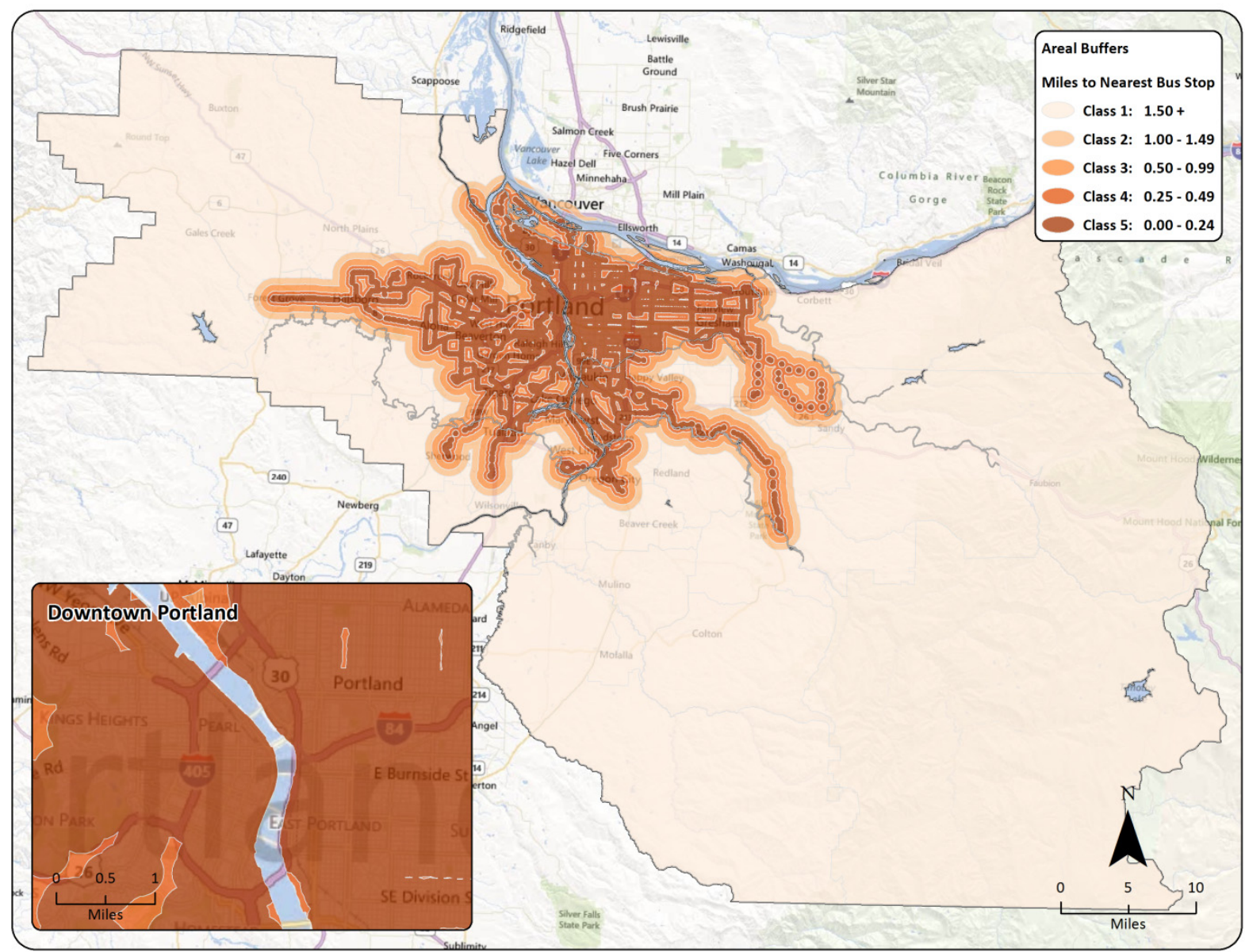

Figure 5-8: Manual classification of straight-line distance to nearest bus stop in Portland metropolitan region

Figure 5-8 is a heat map of the three-county region denoting those areas in which a bus stop may be accessed by one of five straight-line distances that also comprise most chosen distances for the outer-ring radii in this study. As one may imagine, those areas with the closest proximity to a bus stop are centrally located in vicinities with higher population and employment densities. 
Correspondingly, Figure 5-9 reveals those stretches of the Portland metropolitan region that are located within varying levels of proximity to a rail station. The heat map denoting the proximity to a rail station is more dispersed across the study area with a concentration of areas within onequarter mile of a rail station located in downtown Portland. Moreover, a much larger portion of the study area is located outside the areal buffer of 1.5 miles from a rail station in comparison to the amount of the study area classified as being within the same proximity to a bus stop. These differences in the classification of the study area based on distance to nearest bus stop or rail station may illuminate additional or establish previously noted trends regarding the level of anonymity across different contexts of the 25 applications of the donut masking technique

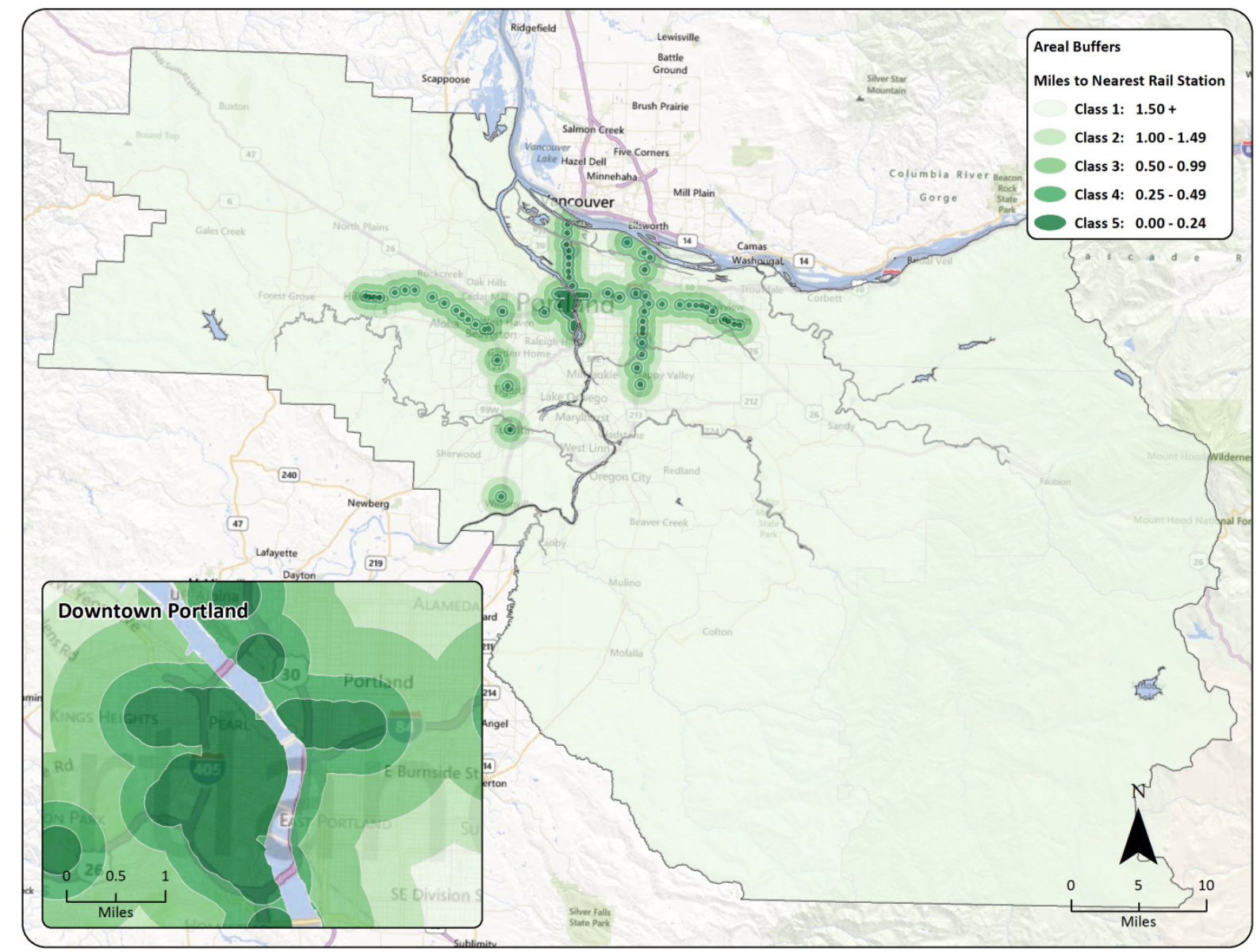

Figure 5-9: Manual classification of straight-line distance to nearest rail station in Portland metropolitan region

In the manner of the previous analyses, Figure 5-10 provides a dot chart of the average median kanonymity statistic of households for the 25 different scenarios, with the anonymity level being divided based on the five manual breaks for the built environment measure of miles to nearest bus stop. Figure 5-11 reflects the same dot chart, but for the classification of anonymity level across different categories of proximity to the nearest rail station. Using disaggregate distance to transit measures for assessing the variation in approximated anonymity levels for different combinations of inner- and outer-ring radii cemented those trends identified in prior divisions of the built environment. 


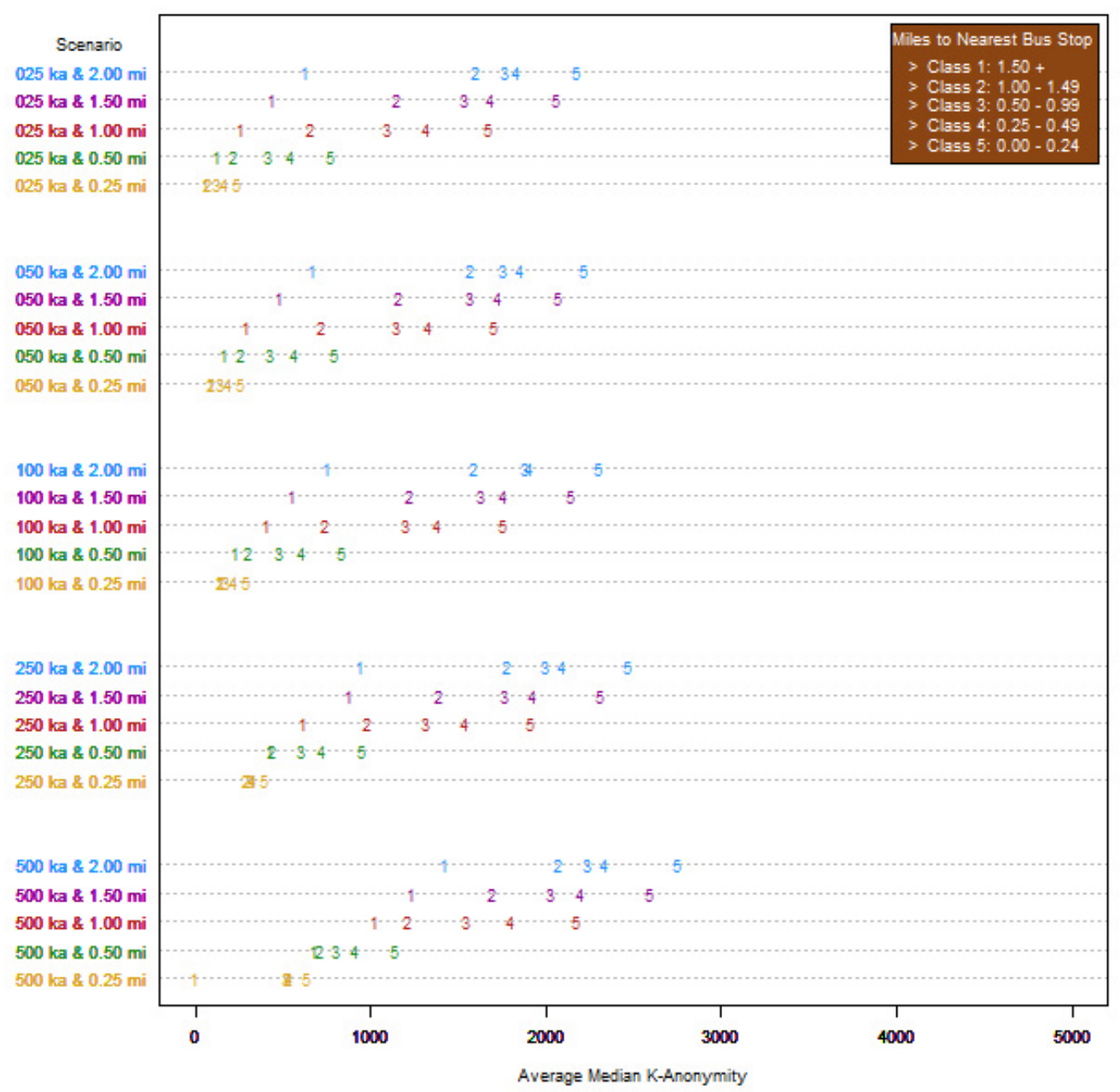

Figure 5-10: Level of anonymity per scenario over 50 simulations (manual breaks for bus stop distance)

First, the average anonymity level increases across scenarios as either the inner or outer ring is extended and the distance of the alternative radius is held constant. Additionally, when only bearing in mind those scenarios in this application, disclosure risk is more efficiently minimized by increasing the potential displacement distance rather than extending the size of the exclusion area. This trend appears to be intrinsic to the chosen methodological approach that defines the inner and outer ring by unrelated strategies. The former radius is identified by a data custodian looking to minimize the risk of identity disclosure, and the latter radius is informed by common practice in studies on non-motorized travel and the built environment. A second trend confirmed from these analyses is the linear rate of increased anonymity associated with those households residing in rural contexts in contrast to the non-linear increase in anonymity for those households located in more urban contexts that diminishes as the outer ring approaches two miles in radius. An assumption that a more urban context is associated with closer proximity to either a bus stop or transit station is made here, but this pattern was identified in the analysis of population density breaks and is likely attributable to the methodological decision to restrict the perturbed location to its original census tract. A third trend, which was disclosed by the use of these disaggregate built environment measures classified on a rural-to-urban spectrum, is that an increase in the average 
anonymity level within each scenario is directly related to an increase in the immediacy of a bus stop or rail station to the household location. This trend is intuitive since minimizing the risk of identity disclosure for a household location is related to a greater displacement distance. However, this hypothesized finding was not clearly evident in the previous analyses utilizing a classification of the built environment with more aggregate area measures. Finally, the maximum average anonymity achievable for scenarios in this donut masking technique application appears to have somewhere around a k-anonymity statistic of 3,000. This anonymity level would seem more than adequate in masking the spatial location of a household in most applications of a household survey dataset. Furthermore, this threshold for minimizing disclosure risk is likely an artifact of the census tract restriction and gives the data custodian an indication of the level of anonymity required for the release of the aggregate U.S. Census dataset.

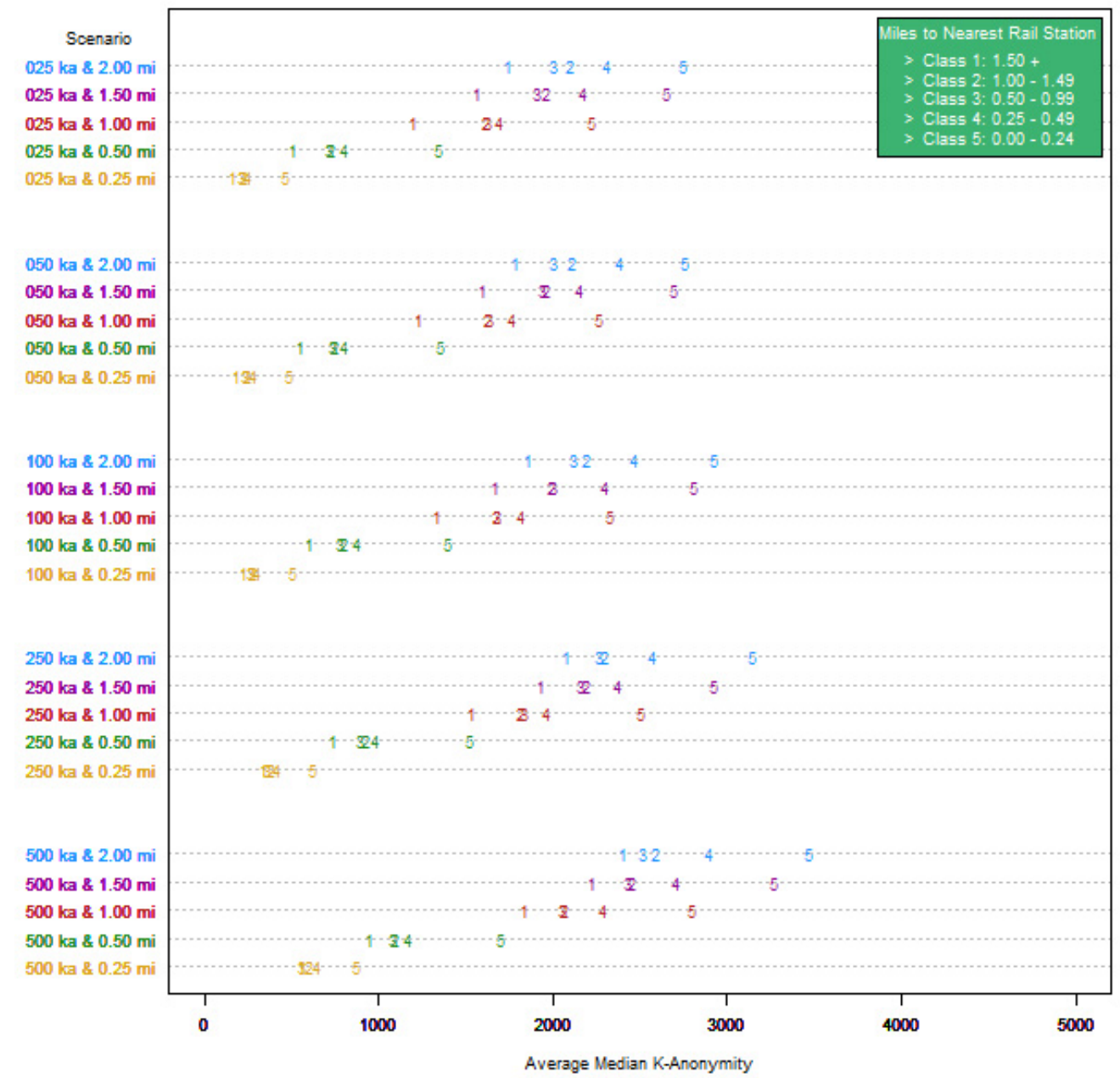

Figure 5-11: Level of anonymity per scenario over 50 simulations (manual breaks for rail station distance)

\subsection{DATA UTILITY ANALYSIS}

The identification of general trends regarding disclosure risk, while a central element for the data custodian to evaluate when spatially perturbing a household location, only reflects half of the alteration necessary in publicly disseminating a disaggregate data source of use to researchers. The 
introduction of spatial error is an accepted condition toward ensuring that the confidentiality of the respondent's identity is preserved when preparing any release of household travel survey data at a finer resolution. However, at some point, the level of spatial error may become too great for the researcher to make any meaningful or assured conclusions from an application of the new dataset. As such, the data custodian should give careful deliberation over the choice of inner- and outerring radii for a particular application of the donut masking technique. A choice to generate a large exclusion area associated with the decreased ability of a data intruder to make any one-to-one correspondence has the added consequence of prohibiting the altered household location of being positioned near its original site. Likewise, the extension of the outer-ring radius, which has been shown to improve the average anonymity level when holding the inner-ring radius constant, permits the geographically perturbed household farther from its true location. The significance of either circumstance is that the displacement of a household farther from its actual location has the heightened potential to position the jittered household into a built environment setting that is not representative of the household's true setting. On the whole, the greater the disparity between the way that these two settings are classified, the less useful the geographically perturbed dataset will become to the researcher.

An assessment into the level of disparity found between the built environment of a household's true location and its spatially perturbed site, across different specifications of the donut masking technique, is found below. This analysis evaluates the average level of spatial error, quantified as PRMSE, for the same five measures of the built environment used in the previous assessment of disclosure risk. Beginning with an aggregate calculation of spatial error across all records for a given scenario, the following analyses eventually move toward an evaluation of spatial error that is based on categorizations of each of these measures of density, design and distance to transit.

Figure 5-12 is a dot chart representing the average level of spatial error introduced in five built environment measures for the 25 applications of the donut masking technique. By quantifying the concept of data utility with the normalized measure of PRMSE, the data custodian is able to compare the spatial error of one built environment measure across different scenarios in addition to the arguably more valuable evaluation of the level of spatial error introduced to various built environment measures within the same scenario. One obvious trend from this aggregate analysis is that an increase in outer-ring radius for the same requirement of anonymity defining the innerring radius produces a greater amount of spatial error in the dataset, no matter what measure of the built environment is examined. Similarly, increasing the inner-ring radius, while maintaining the same outer-ring radius, introduces more spatial error to the dataset. The detection of these trends was predicted and supports the notion that the data custodian must be cognizant of the fact that an increase in the potential displacement distance or exclusion area of an application of the donut masking technique will lead to lower data utility to the researcher. Moreover, of the two methods for changing the donut's size, the level of spatial error introduction increases at greater rate when extending the outer ring from one areal buffer to the next while holding the inner-ring size constant.

A supplement to the identification of aggregate trends based on alterations to the size of either ring of the donut is the recognition of what general trends occur within certain built environment measures when these various scenarios are explored. One general trend evident from Figure 5-12 is that when the outer-ring radius is extended at least one mile from the household location, the order of built environment measures in relation to their level of spatial error is consistent with the 
disaggregate measure of distance to the nearest rail station having the least amount of introduced error and the aggregate measure of employment density having the largest PRMSE values. Also, the order of built environment measures in regard to their spatial error level remains unchanged within an outer-ring grouping when the inner-ring radius is determined by a k-anonymity statistic of 250 or less. The only scenarios when the order of the built environment measures changes in terms of spatial error is when the minimum k-anonymity is increased from 250 to 500 for the onequarter and one-half-mile scenarios. In these two instances, the spatial error for distance to the nearest rail station surpasses the level of introduced error for the measure of population density. As each of these trends assumes the application of a single donut masking technique to the entire dataset without regard for different contexts, this analysis would benefit from an extension of this analysis that examines spatial error across different classifications of the built environment.

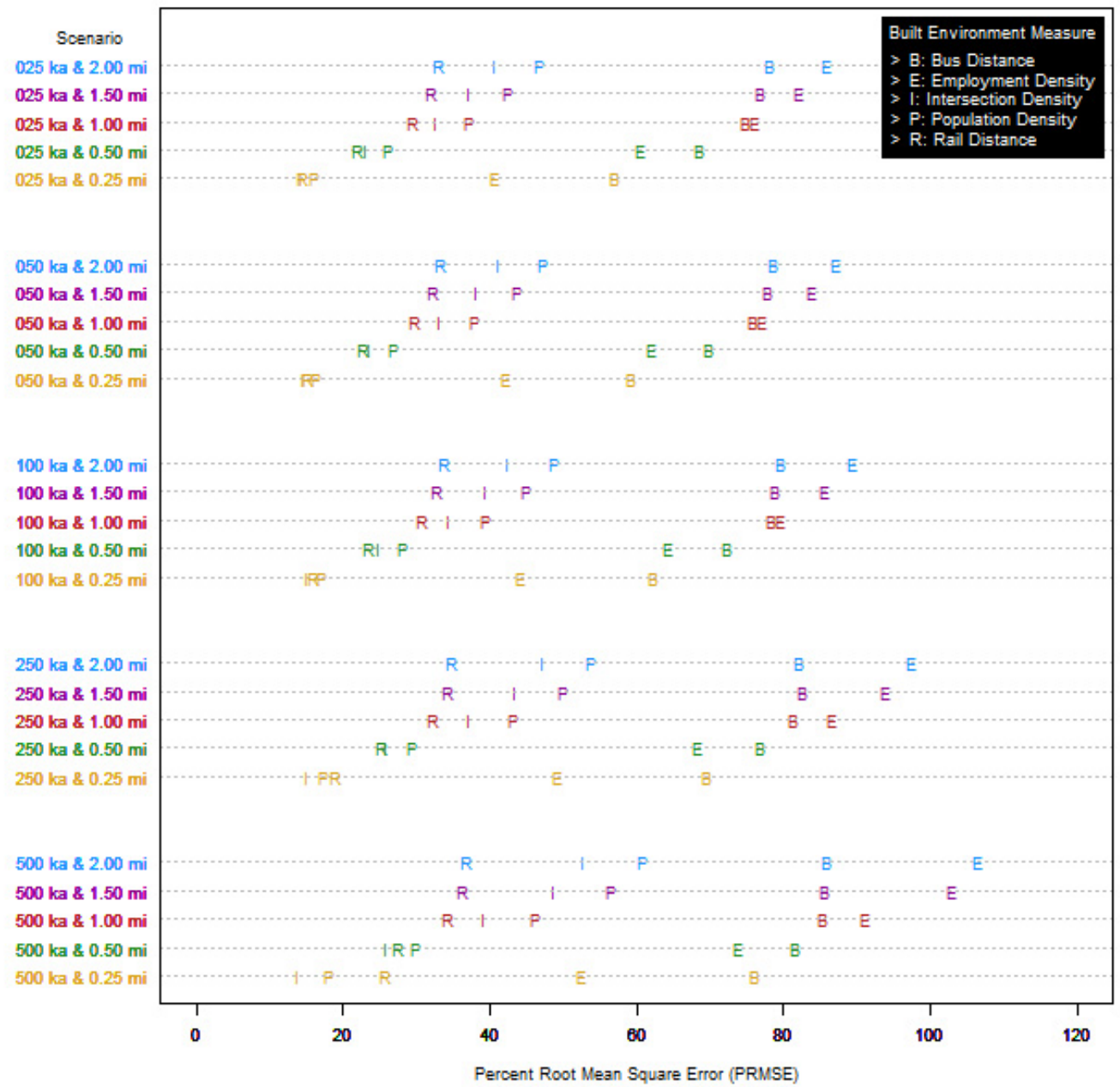

Figure 5-12: Level of spatial error per scenario over 50 simulations (all built environment measures)

\subsubsection{Data Utility within Subcategories of Density Measures}

A more sophisticated approach to disseminating a geographically perturbed dataset would take into account the balance between both concepts of disclosure risk and data utility as they change 
across different spatial contexts. For instance, the data custodian may apply one specification of the donut masking technique to those households located in a rural context, while deciding to apply a more restrictive donut to an urban context where variation in the built environment may be greater. Previous analyses looked at tends in anonymity across these different classifications of the built environment, which in the long run informs the data custodian of what configurations may be ruled out in terms of compromising respondent confidentiality. The following analyses will identify trends in spatial error found across the same built environment contexts, which will in turn provide the data custodian with a better sense of what scenarios provide less informative data to the researcher. Taken together, the understanding of how disclosure risk and data utility vary across different landscapes will enable the data custodian to produce a spatially perturbed dataset balanced in its consideration of anonymity and spatial error.

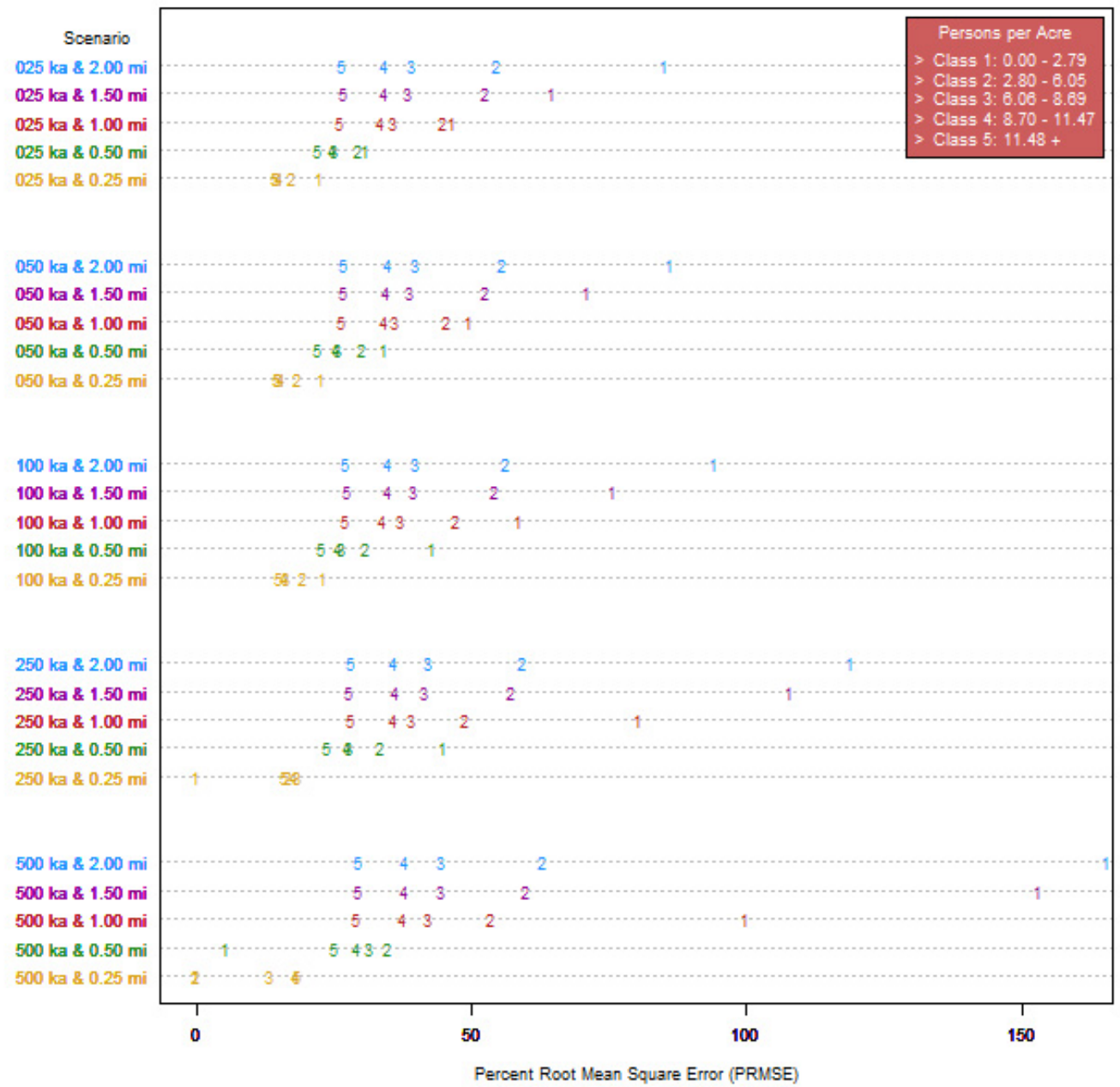

Figure 5-13: Level of spatial error per scenario over 50 simulations (quintiles for population density)

Using the same quintiles displayed in Figure 5-1, Figure 5-13 provides an overview of the level of spatial error found within different categorizations of population density for the 25 scenarios explored in this study. Overall, the geographic perturbation of households located in the lowest quintile of population density produces the greatest level of spatial error in those scenarios where 
the outer-ring radius is at least one mile in distance. Moreover, in each of the scenarios defined by an outer-ring radius of at least one mile, the level of spatial error increases as the context of the household becomes more rural in nature. A handful of other trends that have parallels with the discussion on anonymity were also true in this investigation of data utility. One trend was that the rate of increase in PRMSE value was slower in the more urban contexts for scenarios in which the inner-ring radius was held constant and the outer-ring radius was allowed to increase. For households located in rural contexts, this increase in outer-ring radius for scenarios grouped by a comparable inner-ring specification led to a much greater rate of deterioration in the utility of the perturbed dataset. Additionally, the introduction of spatial error leveled off for households in the densest quintile when the outer-ring radius was at least one mile in distance for a particular scenario.

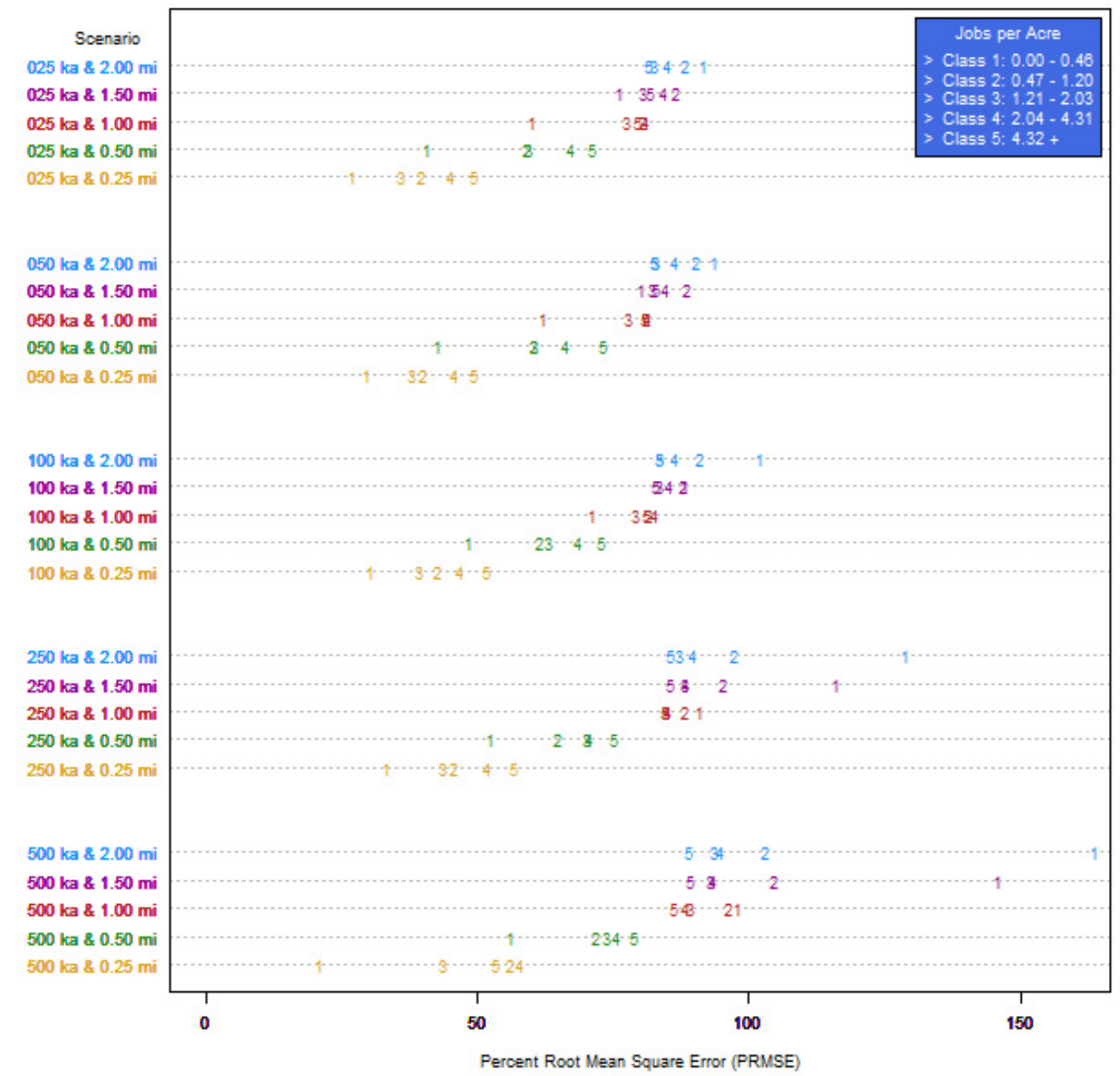

Figure 5-14: Level of spatial error per scenario over 50 simulations (quintiles for employment density)

Figure 5-14 provides a complementary illustration of how spatial error varies across the different groupings of employment density within a given arrangement of inner and outer rings. While in many ways this analysis reveals the same trends as the above assessment, this analysis into the quintile division of tracts by employment density does reveal one unique trend concerning spatial error. For those scenarios where the outer-ring radius was set to be less than one mile in distance, 
households located in tracts with the lowest level of employment density had the least amount of error introduced in spatially perturbing their location. Households residing in tracts characterized by the highest level of employment density had the greatest amount of spatial error once their location was geographically perturbed. However, for the scenarios in which the outer-ring radius was specified to be 1.5 miles or greater, those households in the quintile with the lowest employment density now had the greatest amount of spatial error introduced when repositioned, while the households located in the tracts with the densest level of employment had the lowest PRMSE. The lone exception to this trend is found in the scenario where the inner ring was defined by the highest level of disclosure risk and a maximum displacement distance of 1.5 miles. Additionally, spatial error introduction increases at a greater rate for those households that are located in tracts characterized by a lower employment density. Overall, there is generally less variation when a respondent's household location has been geographically perturbed within a tract characterized by high employment density.

\subsubsection{Data Utility within Subcategories of Design Measures}

The following subsection examines how data utility varies across different gradations of a third areal measure of the built environment, intersection density. Akin to the evaluation of anonymity over 25 specifications of the donut masking technique, this operationalization of data utility as PRMSE looks at how the repositioning of a household changes the quantification of the concept across one of five natural break classifications of the respondent's census tract. Figure 5-6 earlier displayed the distribution of these intersection density classifications of census tracts across the three-county study setting. Similar to the density measure for population, many of those tracts characterized by the highest level of intersection density are centrally located within Portland. As such, households located in census tracts with a higher intersection density can be thought of as residing in a more urban context. Understanding how spatial error introduction varies across such regional contexts given a certain combination of inner- and outer-ring sizes will help to inform what specification a data custodian may select in order to provide the researcher with an altered dataset that is useful in its representation of this particular design feature.

A glance at the aggregate patterns across all built environment measures showed that the utility of intersection density was better preserved than either population or employment density when the household was geographically perturbed. The PRMSE value only exceeded 50 when the inner ring was generated with a k-anonymity statistic of 500 and the outer-ring radius extended out to two miles from the actual household location. Additionally, the range of PRMSE values within scenarios of a given outer-ring radius was relatively small compared to the PRMSE values for other density built environment measures. Having established these general trends associated with a blanketed look at spatial error introduction for different scenarios, the dot chart of Figure 5-15 provides additional insight into more nuanced trends in data utility for households residing in tracts defined by one of five subcategories of intersection density. 


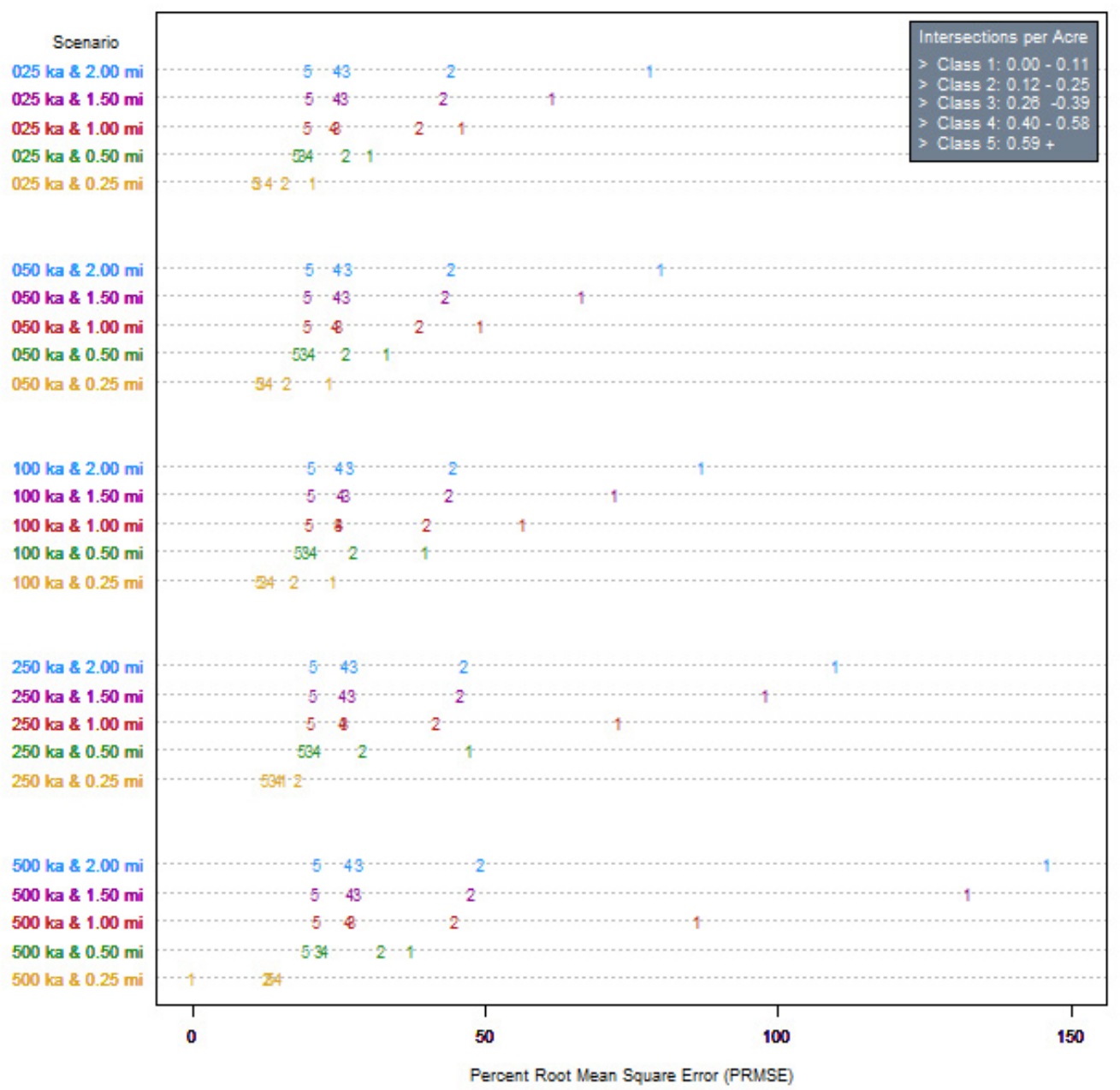

Figure 5-15: Level of spatial error per scenario over 50 simulations (natural breaks for intersection density)

The behavior of this design measure in relation to the amount of spatial error introduced to an altered dataset by any specification of the donut masking technique is largely consistent across the diverse classifications. For the scenarios with an outer ring of at least one-half mile, the more rural the context of the survey respondent and the more spatial error introduced when altering their household location. This tendency is only challenged in those two scenarios where the exclusion area is defined by an estimated k-anonymity statistic of 250 or 500 and the outer-ring radius is only extended one-quarter of a mile from the location of households in the dataset. This finding hints at the earlier trend identified in the employment density analysis in which the level of error reversed when the configuration of the outer ring of the donut extended beyond one mile, but more likely may be related to a circumstance in which the repositioned household is placed within its original block group. This would produce no spatial error in this aggregate measure. Furthermore, while the range of PRMSE values for intersection density was small relative to other density measures when looking at error across an aggregation of landscapes, this closer examination of PRMSE reveals that the range of spatial error introduction was much wider for households located in census tracts characterized as having less than 0.26 intersections per acre. In fact, those households in the most rural context had a PRMSE value greater than 50 for this measure of urban 
design when allowing their household to be potentially repositioned at least 1.5 miles from its original location.

\subsubsection{Data Utility within Subcategories of Distance to Transit Measures}

The previous analysis of the level of variation introduced into a dataset by altering the original position of a household and measuring the built environment focused on disaggregate measures. While this prior analysis of density and design measures was valuable in assessing how the use of certain donut masking technique specifications influenced the utility of the altered dataset, there is additional insight to be gained through an examination of disaggregate built environment measures. One clear benefit from such an evaluation is an understanding that some spatial error is introduced to a dataset whenever the original household location has been spatially perturbed. In the previous assessment of spatial error introduction pertaining to area-based measures, there was the chance that a donut masking technique would reposition a household to a new location without any change in the built environment measure. Although such a circumstance could occur when evaluating variation based on a single built environment measure, the reason for this lack of error in these described instances was due to the aggregate depiction of the built environment. Such a representation of the built environment allowed a household to potentially be repositioned within its home census block group. When this event occurred during any of the 50 simulations of a household for a particular scenario, the relative error in the built environment was measured as zero. Given the geographic size of block groups is larger for those administrative units located in less populated areas, the aggregate nature of these measures may have led to situations where the use of a restricted donut in perturbing the location of households in this context has small PRMSE values. For this reason, an evaluation of data utility for a disaggregate built environment measure across various classifications of the study area is a necessary exercise. Furthermore, the understanding of how built environment measures with a higher resolution are influenced by the implementation of different scenarios of the donut masking technique is paramount in avoiding any ecological fallacy in examining the relationship between non-motorized travel and the built environment.

The two disaggregate built environment measures used in this analysis of the utility of an altered dataset are the distance to the nearest bus stop and distance to the nearest rail station. Figure 5-16 provides a dot chart reflecting the level of spatial error per scenario for the accessibility measure of distance to nearest bus stop across five classifications for proximity informed by the literature. A trend evident from this visualization is that the order of the classifications is not successive in any of the scenarios. While the PRMSE values associated with households residing farther than 1.5 miles from the nearest bus stop are the lowest in each scenario, there may be some surprise in the finding that PRMSE values for the fourth classification, and not the fifth, are the greatest in each application of the donut masking technique with an outer ring of at least one-half mile. In four of the five scenarios where the outer-ring radius is one-quarter of a mile in length, the order of classifications was sequential with those households located closest to a bus stop having the most variation introduced to the dataset when spatially repositioning their residence. Another trend in regard to the data utility of this distance to transit measure was that the PRMSE values for households located within one-half mile straight-line distance from a bus stop were fairly stable for those scenarios in which the maximum potential displacement distance was at least one mile. This latter finding is likely related to the joint influence of the methodological approach 
constraining the repositioned household to remain within its original tract and the nature of the built environment measure being a straight-line calculation connecting two points on a map.

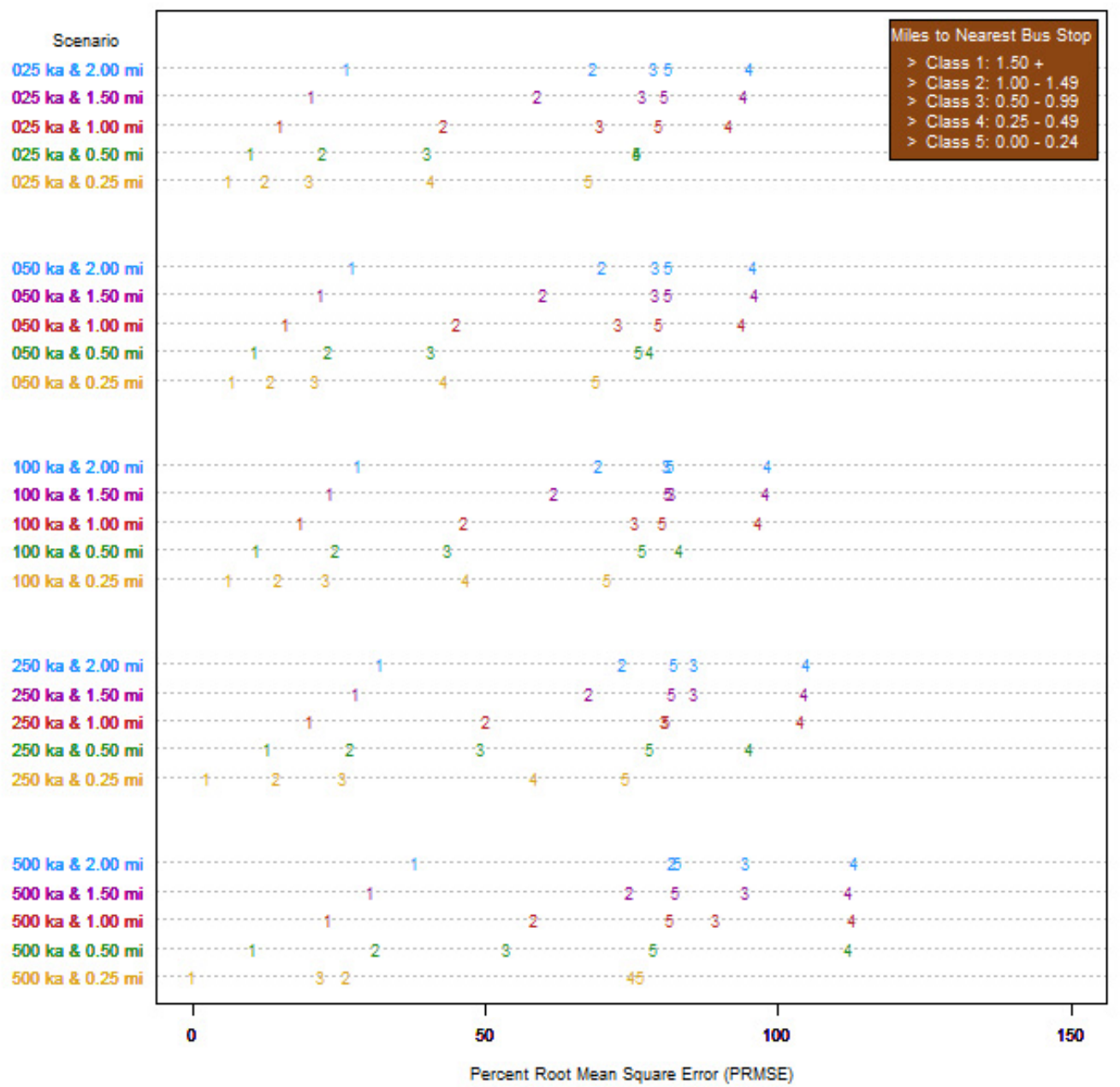

Figure 5-16: Level of spatial error per scenario over 50 simulations (manual breaks for bus stop distance)

This link between the use of a straight-line calculation for a distance to transit measure and the application of the donut masking technique becomes clearer when looking at the distribution of PRMSE values for the manual classifications of the miles to nearest rail station measure. Figure 5-17 provides the familiar dot chart of introduced spatial error values per classification for this disaggregate measure of the built environment across the 25 different configurations of the donut masking technique. Expectedly, there was little change in the level of spatial error introduced to the altered dataset resulting from an increased level of anonymity defining the exclusion area. In fact, the spacing of PRMSE values between the categories in the specification resulting in the smallest inner-ring radius and largest outer-ring radius were fairly uniform. In contrast, the gaps in PRMSE values for the scenario defined by the largest k-anonymity statistic and smallest outerring distance were clustered when the households were originally located less than one mile from the nearest rail station. Overall, the behavior of this particular disaggregate distance to transit measures is well understood with the outcome of introduced spatial error in the altered dataset 
ultimately dependent upon the specification of the donut masking technique designated by the data custodian.

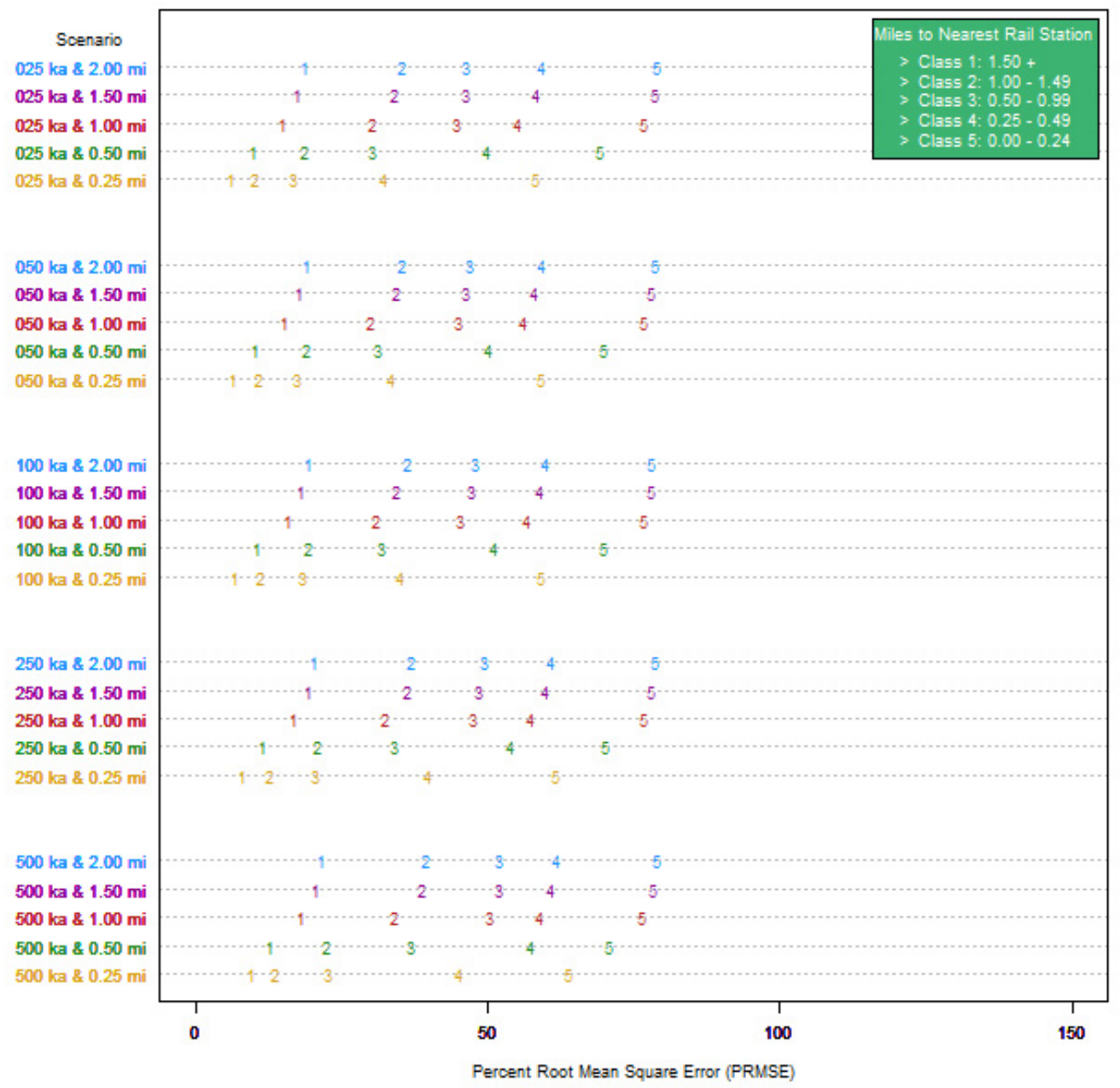

Figure 5-17: Level of spatial error per scenario over 50 simulations (manual breaks for rail station distance) 


\subsection{DISCUSSION}

This exploration of an innovative technique to spatially perturb the residences of respondents to a household travel survey in the Portland metropolitan region has unveiled a number of common themes regarding disclosure risk, data utility and data retention. The concept of disclosure risk examined in this study denotes the ability of a data intruder to make a one-to-one correspondence between the location of the geographically perturbed household and their actual residence with relatively little effort and without any additional data sources or external knowledge of the survey respondent. An evaluation of this concept was undertaken through the estimation of a k-anonymity statistic found in the information sciences, and was operationalized in this technique by the creation of an exclusion area in which the perturbed household cannot be geographically repositioned. The complementary concept of data utility refers to the introduction of spatial error to a dataset that occurs when the location of household respondents have been altered through the implementation of the donut masking technique. Data utility was assessed by measuring the PRMSE of five built environment measures as they relate to the actual and perturbed household locations. This calculation of spatial error introduction was strongly connected to the selection of outer-ring radius distances, which was largely contingent on areal buffers commonly studied in research on the links between non-motorized travel and the built environment. The balancing of this preservation of data utility and minimization of disclosure risk is ultimately determined by the choice of inner and outer rings, which will in turn advise the data custodian of the degree of records that can be retained by the chosen specification. Collectively, these themes are central to the public dissemination of these disaggregate data and help to inform the data custodian in her choice of a suitable specification of the donut masking technique, which both protects the confidentiality pledge with the survey respondent and maintains the utility of the dataset to the researcher.

An improved understanding of the interconnected nature of these three concepts was established through an empirical application of the donut masking technique with 25 unique specifications. The themes collected from this analysis offer several rules of thumb to be considered in any future application of this geographic perturbation technique as well as a number of thoughts that may guide future extensions of this research. The following chapter describes several of these guidelines in addition to noting some limitations and potential future expansions of this research.

\subsection{GENERAL RULES OF THUMB FOR FUTURE APPLICATION}

Having conducted an empirical application of the donut masking techniques using household travel survey data from the Portland metropolitan area, several trends were uncovered regarding the interplay between data retention, disclosure risk and data utility. The choice of specification for the application of this geographic perturbation technique had repercussions for each of these themes that, when taken together, provide some insight for any future application. The following bulleted points outline some general rules of thumb that may be taken away from this effort.

- The ideal balance of minimum disclosure risk and maximum data utility should only be sought after in the most urban of contexts, as the configuration of such a donut for a less densely populated area will result in the significant loss of sampled observations. 
- For the greatest improvement in average levels of anonymity, the maximum displacement distance should be extended; however, the return on such an extension is less beneficial to a household in an urban context after one mile.

- An improvement in the utility of the dataset with regard to employment density of the respondent's census block group should be sought since this built environment measure was most impacted by the geographic perturbation of the actual household location.

- When seeking to maximize the utility of density measures of the built environment, the data custodian should be cognizant that the specification of a scenario with a maximum displacement distance over one mile may introduce excessive error in rural contexts.

- To provide an altered dataset giving adequate attention to both concepts of disclosure risk and data utility, the single specification of a donut masking technique to all respondents in the dataset should be avoided by a data custodian.

While many of these points are an artifact of this specific implementation of the donut masking technique, they do help to establish some clear starting points for any potential future expansion. Of course, being that the points are rough guidelines related to this particular application, any extension of this work should not completely rest upon these suggestions.

\subsection{LIMITATIONS AND POTENTIAL FUTURE EXPANSIONS}

This study has shown the promise of the donut masking technique in negotiating the balance between disclosure risk and data utility that is central to the distribution of a household travel survey dataset with heightened spatial resolution. Nevertheless, the exploratory nature of this application of a new geographic perturbation technique has both provided valuable insight to researchers and raised questions to be addressed by any future expansion of this donut masking technique. The limitations of this empirical application are related to the selected methodological approach, which were difficult to foresee prior to implementation due to the innovative nature of this research, in addition to the defined scope of work. The scope strictly focused on the prevention of a first-tier breach in the confidentiality pledge between the survey respondent and collector of the survey data. Moreover, there exist plenty of broader themes concerning the complete release of a disaggregate data source for public consumption that warrant contemplation by the travel survey community. While in no way an exhaustive list, some of these themes are also identified in the following discussion.

In relation to the methodology selected for this application, there was a necessity in recognizing the number of records preserved by the specification of the donut masking technique due to the lack of interdependency between the inner- and outer-ring radii. In this application, the distance of the inner-ring radius for each household is dependent on the level of anonymity desired by the data custodian. Thus, the exclusion area's size is tied to the residential density of the household's census tract, where households located in urban contexts have a minimum displacement distance that is smaller than their rural counterparts. A household in a densely populated census tract does not have to be repositioned as far as a household in a sparsely populated tract in order to provide the same estimated k-anonymity statistic. On the other hand, the selection of an outer-ring radius was a manual specification of distance that was informed by practices common to the literature. Accordingly, there were certain scenarios in which a higher level of anonymity required some 
households to be relocated a distance greater than the maximum displacement distance defined. This circumstance resulted in the failure to reposition a household within any geographic space due to the collapsing of the donut. In these instances, the data custodian must consider the value of retaining these records in the altered dataset and proceed accordingly.

Another methodological limitation to be addressed in future applications relates to the restriction of the household to their original census tract. This constraint is related to the use of residential density at the census tract geography in estimating the k-anonymity statistic. There is the potential for future expansions to use disaggregate household positions in calculating this measure of disclosure risk that would not confine the repositioned household to this administrative boundary, which could subsequently help the retention of data records since the outer-ring perimeter would often be extended in those scenarios with a greater maximum displacement distance. Related to this request for a disaggregate measure of household location, which was not pursued because of computational burden and lack of study area coverage in such a data source, is the exploration of data utility using additional built environment measures with improved resolution. In this study, the only truly disaggregate data were classified as distance to transit measures. Future expansions should examine the relationship between different scenarios and the utility of a count or density measure that is not aggregated to the census block group. Such measures have the potential to be more informative in studies on non-motorized travel as they will address ecological fallacy concerns that have clouded many past research efforts.

In addition to these methodological limitations, there were a number of directions that should be explored by future geographic perturbation applications. For instance, the scope of this study was restricted to the understanding of how the geographic perturbation of household locations relates to the reduction in first-tier disclosure risk. While the importance of lessening the likelihood of this highest breach in respondent confidentiality was explored in this application, a thorough examination of disclosure risk must also reduce the prospect of any second- or third-tier breach. The spatial perturbation of activity locations, which would also diminish the utility of a dataset, must also be considered by a data custodian in order to publicly disseminate these valuable data. Information regarding a household member's employment location may provide the additional piece of knowledge required of a data intruder to make a one-to-one correspondence. Thus, the data custodian must spatially perturb the activity location in addition to their residence so as to preserve survey respondent anonymity within the disaggregate dataset. Furthermore, additional consideration may be given by the data custodian to provide further practicality to the altered dataset by only allowing the randomly perturbed household or activity location to be placed in a corresponding zoning district. In addition to this constraint of land use preservation, which may help to limit the introduction of spatial error in regard to the built environment, is the notion of data swapping. The random spatial repositioning of a household unit has the potential to place the geographically perturbed location on the site of another household unit, raising concerns regarding the misidentification of one household and their attributes as another household in the release of an altered dataset.

This last illustration reflects one of the many challenges of using the donut masking technique as a post-processing strategy used for publicly releasing these disaggregate data. As travel survey data collection methods continue to move away from traditional approaches and toward real-time strategies, there is an increased requirement on the behalf of the data custodian to assure survey 
respondents that their data remain confidential. This instillation of confidence in the respondent becomes more difficult as the data custodian begins to consider the external knowledge of data intruders or the resources available to them. In the past, a sunset period has been assumed to exist on such data as household and travel attributes are assumed to change over a given period of time. However, the increased ability to spread household travel data almost instantaneously because of technological advancement has forced participants in the data collection process to consider the true risk of disseminating these data. Obviously, a greater discussion concerning the proper level of anonymity must be given further thought by the profession. This exploration has looked to advance such deliberation through the empirical application of a spatial perturbation technique aimed at examining the inherent tradeoff between disclosure risk and data utility when seeking to disseminate disaggregate household travel survey data. 


\subsection{REFERENCES}

Allshouse, W. et al. Geomasking sensitive health data and privacy protection: An evaluation using an E911 database. Geocarto International, Vol. 25, No. 6, 2010, pp. 443-452.

An, M. and M. Chen. Estimating nonmotorized travel demand. In Transportation Research Record: Journal of the Transportation Research Board, No. 2002, Transportation Research Board of the National Academies, Washington, DC, 2007, pp. 18-25.

Armstrong, M., G. Rushton, and D. Zimmerman. Geographically masking health data to preserve confidentiality. Statistics in Medicine, Vol. 18, 1999, pp. 497-525.

Badoe, D. and E. Miller. Transportation-land use interaction: Empirical findings in North America, and their implications for modeling. Transportation Research Part D, Vol. 5, 2000, pp. 235-263.

Bhat, C. and H. Zhao. The spatial analysis of activity stop generation. Transportation Research Part B, Vol. 36, 2002, pp. 557-575.

Cassa, C., S. Grannis, J. Overhage, and K. Mandl. A context-sensitive approach to anonymizing spatial surveillance data: Impact on outbreak detection. Journal of the American Medical Informatics Association, Vol. 13, No. 2, 2006, pp. 160-165.

Cervero, R. Alternative approaches to modeling travel-demand impacts on smart growth. Journal of the American Planning Association, Vol. 72, No. 3, 2006, pp. 285-295.

Cervero, R. and K. Kockelman. Travel demand and the 3Ds: Density, diversity, and design. Transportation Research Part D, Vol. 2, No. 3, 1997, pp. 199-219.

Chatman, D. Residential choice, the built environment, and nonwork travel: evidence using new data and methods. Environment and Planning A, 2009, Vol. 41, pp. 1072-1089.

Chen, C., H. Gong, and R. Passwell. Role of the built environment on mode choice decisions: additional evidence on the impact of density. Transportation, Vol. 35, No. 3, pp. 285-299.

Chikaraishi, M., A. Fujiwara, J. Zhang, and K. Axhausen. Exploring variation properties of departure time choice behavior by using multilevel analysis approach. In Transportation Research Record: Journal of the Transportation Research Board, No. 2134, Transportation Research Board of the National Academies, Washington, DC, 2009, pp. 10-20.

Clifton, K. (corresponding author), Burnier, C., and G. Akar. Severity of injury resulting from pedestrian-vehicle crashes: What can we learn from examining the built environment? Transportation Research Part D, Vol. 14, 2009, pp. 425-436.

Clifton, K. (corresponding author) and N. Noyan. Framework for applying data masking and geoperturbation methods to household travel survey datasets. Presented at $91^{\text {st }}$ Annual Meeting of Transportation Research Board, Washington, D.C., 2012. 
Curtis, A., J. Mills, L. Agustin, and M. Cockburn. Confidentiality risks in fine scale aggregations of health data. Computers, Environment and Urban Studies, Vol. 35, 2011, pp. 57-64.

Dalenius, T. and S. Reiss. Data-swapping: A technique for disclosure control. Journal of Statistical Planning and Inference, Vol. 6, 1982, pp. 73-85.

Davidson, W., R. Donnelly, P. Vovsha, J. Freedman, S. Ruegg, J. Hicks, J. Castiglione, and R. Picado. Synthesis of first practices and operational research approaches in activity-based travel demand modeling. Transportation Research Part A, Vol. 41, 2007, pp. 464-488.

Domingo-Ferrer, J. and V. Torra. Disclosure risk assessment in statistical data protection. Journal of Computational and Applied Mathematics, Vol. 164-165, 2004, pp. 285-293.

Duke-Williams, O. and P. Rees. Can census offices publish statistics for more than one small area geography? An analysis of the differencing problem in statistical disclosure. International Journal of Geographic Information Science, Vol. 12, No. 6, 1998, pp. 579-605.

Duncan, G. and D. Lambert. The risk of disclosure for microdata. Journal of Business and Economic Statistics, Vol. 7, No. 2, 1989, pp. 207-217.

Ewing, R. and R. Cervero. Travel and the built environment: A meta-analysis. Journal of the American Planning Association, Vol. 76, No. 3, 2010, pp. 265-294.

Fellegi, I.P. On the question of statistical confidentiality. Journal of the American Statistical Association. Vol 67. No. 337, 1972, pp. 7-18.

Frank, L. and G. Pivo. Impacts of mixed use and density on utilization of three modes of travel: Single-occupant vehicle, transit, and walking. In Transportation Research Record: Journal of the Transportation Research Board, No. 1466, Transportation Research Board of the National Academies, Washington, DC, 1994, pp. 44-52.

French, J. and M. Wand. Additive models for cancer mapping with incomplete covariates. Biostatistics, Vol. 5, No. 2, 2004, pp. 171-191.

Fuller, W. Masking procedurse for microdata disclosure limitation. Journal of Official Statistics. Vol. 9, 1993, pp. 383-406.

Goulias, K. and T. Kim. Multilevel analysis of activity and travel ratterns: Accounting for personand household-specific observed and unobserved effects simultaneously. In Transportation Research Record: Journal of the Transportation Research Board, No. 1752, Transportation Research Board of the National Academies, Washington, DC, 2001, pp. 23-31.

Greenwald, M. and M. Boarnet. Built environment as determinant of walking behavior: Analyzing nonwork pedestrian travel in Portland, Oregon. In Transportation Research Record: Journal of 
the Transportation Research Board, No. 1780, Transportation Research Board of the National Academies, Washington, DC, 2001, pp. 33-42.

Gutmann, M., et al. Providing spatial data for secondary analysis: Issues and current practices Relating to Confidentiality. Population Research and Policy Review, Vol. 27, 2008, pp. 639-665.

Hampton, K. et al. Mapping health data: Improved privacy protection with donut method geomasking. American Journal of Epidemiology, Vol. 172, No. 9, 2010, pp. 1062-1069.

Handy, S., M. Boarnet, R. Ewing, and R. Killingsworth. How the built environment affects physical activity: Views from urban planning. American Journal of Preventive Medicine, Vol. 23, 2S, 2002, pp. 64-73.

Karr, A., et al. A framework for evaluating the utility of data altered to protect confidentiality. The American Statistician, Vol. 60, No. 3, 2006, pp. 224-232.

Krizek, K. Operationalizing neighborhood accessibility for land use-travel behavior research and regional modeling. Journal of Planning Education and Research, Vol. 22, 2003, pp. 270-287.

Kwan, M.P., I. Casa, and B. Schmitz. Protection of geoprivacy and accuracy of spatial information: How effective are geographical masks? Cartographica, Vol. 39, No. 2, 2004, pp. 15-28.

Lee, C. and A. Vernez Moudon. The 3Ds + R: Quantifying land use and urban form correlates of walking. Transportation Research Part D, Vol. 11, No. 3, 2006, pp. 204-215.

Leitner, M. and B. Buttenfield. Guidelines for the display of attribute certainty. Cartography and Geographic Information Science, Vol. 27, No. 1, 2000, pp. 3-14.

Leitner, M. and A. Curtis. A First step towards a framework for presenting the location of confidential point data on maps- results of an empirical perceptual study. International Journal of Geographic Information Science, Vol. 20, No.7, 2006, pp. 813-822.

McGinn, A., K. Evenson, A. Herring, S. Huston, and D. Rodriguez. Exploring associations between physical activity and perceived and objective measures of the built environment. Journal of Urban Health, Vol. 84, No. 2, 2007, pp. 162-184.

Olson, K., S. Grannis, and K. Mandl. Privacy protection versus cluster detection in spatial epidemiology. American Journal of Public Health, Vol. 96, No. 11, 2006, pp. 2002-2008.

Oregon Modeling Steering Committee, 2009-2011 Oregon Travel and Activity Survey; URL: http://www.oregon.gov/ODOT/TD/TP/pages/travelsurvey.aspx

Paass, G. Disclosure risk and disclosure avoidance for microdata. Journal of Business and Economic Statistics. Vol. 6, No. 4, 1988, pp. 487-500. 
Rodriguez, D. and J. Joo. The relationship between non-motorized mode choice and the local physical environment. Transportation Research Part D, Vol. 9, 2004, pp. 151-173.

Rushton, G. Geocoding in cancer research: A review. American Journal of Preventive Medicine, Vol. 30, 2S, 2006, pp. 16-24.

Rushton, G., et al. Analyzing geographic patterns of disease incidence: rates of late-stage colorectal cancer in Iowa. Journal of Medical Systems, Vol. 28, No. 3, 2004, pp. 223-236.

Rushton, G., et al. Geocoding Health Data: The use of geographic codes in cancer prevention and control, research, and practice. CRC Press, Boca Raton, FL, 2008.

Schlossberg, M. J. Greene, and P. Phillips. School trips: Effects of urban form and distance on travel mode. Journal of the American Planning Association, Vol. 72, No. 3, 2006.

Susilo, Y.. K. Williams, M. Lindsay, and C. Dair. The influence of individuals' environmental attitudes and urban design features on their travel patterns in sustainable neighborhoods in UK. Transportation Research Part D, Vol. 17, 2012, pp. 190-200.

Sweeney, L. K-Anonymity: A model for predicting privacy. International Journal of Uncertainty, Fuzziness and Knowledge-Based Systems, Vol. 10, No. 5, 2002, pp. 557-570.

Targa, Felipe and Clifton, Kelly J. (corresponding author). "The built environment and trip generation for non-motorized travel," Journal of Transportation and Statistics, Vol. 8, No.3, 2005. pp. 55-70.

VanWey, L., et al. Confidentiality and spatially explicit data: Concerns and challenges. Proceedings of the National Academy of Sciences of the United States of America, Vol. 102, No. 43, 2005, pp. 15337-15342.

Young, C., D. Martin, and C. Skinner. Geographically intelligent disclosure control for flexible aggregation of census data. International Journal of Geographic Information Science, Vol. 23, No. 4, 2009, pp. 457-482.

Zimmerman, D. and C. Pavlik. Quantifying the effects of mask metadata disclosure and multiple releases on the confidentiality of geographically masked health data. Geographical Analysis, Vol. 40, 2008, pp. 52-76. 


\section{APPENDICES}

\section{APPENDIX A: DONUT MASKING TECHNIQUE POSTGIS CODE}

\section{$\underline{\text { README }}$}

Requirements:

Postgres 9.2 with PostGIS

Order of code to be run, plus brief description.

1.jitter_load_data.sql

Executed by user.

Loads shapefiles into postgres tables, converts everything to common spatial reference.

2. jitter_setupfunctions.sql

Executed by user

Two purposes:

- creates postgres functions that do the work.

- calls the functions to do the work of generating jittered scenario points.

Loads the following files as functions.

2.a create_function_jittery_calculate_inner_radius.sql

Imported by jitter_setupfunctions.sql

Calculates the inner-radius diameter based on $\mathrm{HH}$ counts.

2.b create_function_jittery_make_donuts.sql

Imported by jitter_setupfunctions.sql

Creates donut shaped geometry

2.c create_function_jittery_random_point_nulls_handled.sql

Imported by jitter_setupfunctions.sql

Generates random points

2.d.i create_function_jittery_boston_nearest_neighbor.sq1

2.d.ii create_function_jittery_boston_call_nn.sql

2.d.iii create_function_jittery_boston_expand.sql

2.d.iv create_function_jittery_get_nn_to_sample_or_iteration.sql

Imported by jitter_setupfunctions.sql

Finds the nearest neighbor point between two sets

2.e create_function_jittery_generate_scenarios_and_counts.sql

Imported by jitter_setupfunctions.sql

Main function that executes the work of generating scenarios \& counts.

2.f create_function_jittery_median.sql

Imported by jitter_setupfunctions.sql

Generates a median from a list of numbers.

3. runit.sh

Executed by user (unix environment only)

Exports the scenario data.

3.a template_export.sql

Helper script used in runit.sh 
jitter_load_data.sql

create schema jittery;

-- These data are in feet

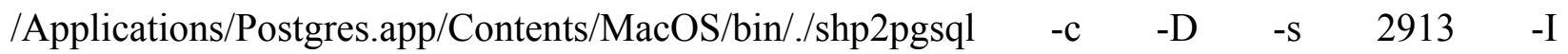
data_to_import/BE_Intersection jittery.be_i $\mid$ psql -d omc $-\mathrm{h}$ localhost

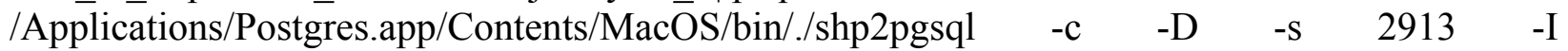
data_to_import/Geography_BlockGroup jittery.geo_bg | psql -d omc -h localhost

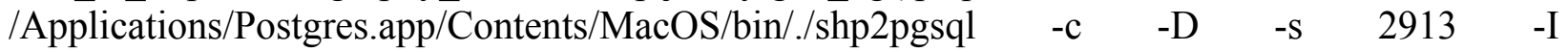

data_to_import/Geography_Tract jittery.geo_tr $\mid$ psql -d omc -h localhost

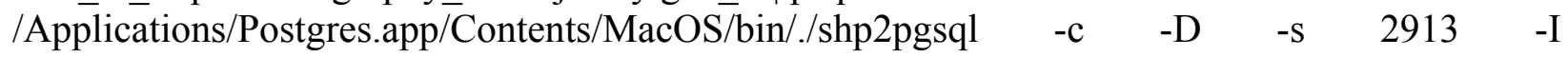
data_to_import/OHAS_Household jittery.ohas | psql -d omc -h localhost

--These data are in utm

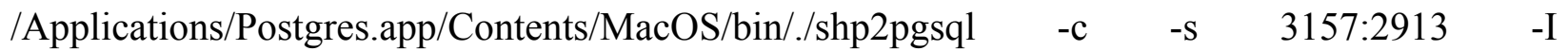

data_to_import/BE_TransitRail jittery.be_r $\mid$ psql -d omc -h localhost

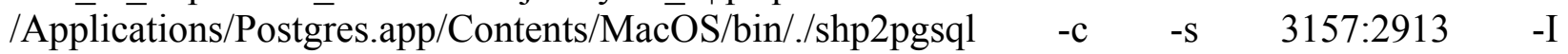

data_to_import/BE_TransitBus jittery.be_b| psql -d omc $-\mathrm{h}$ localhost

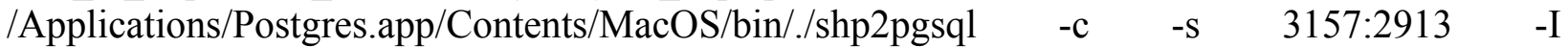

data_to_import/intersection_density jittery.int_dens $\mid$ psql -d omc -h localhost

jitter_setupfunctions.sql

-- Set up the schema for the functions to live

drop schema if exists jit_functions CASCADE;

create schema jit_functions;

-- Type is used in the function: create_function_jittery_boston_call_nn.sql

-- If need to alter name or location, make sure that function is updated.

DROP TYPE IF EXISTS pgis_nn;

CREATE TYPE pgis_nn AS

(gid integer, dist numeric $(12,5)$ );

--import the functions

li create_function_jittery_calculate_inner_radius.sql

li create_function_jittery_make_donuts.sql

li create_function_jittery_random_point_nulls_handled.sql

i create_function_jittery_boston_nearest_neighbor.sql

li create_function_jittery_boston_call_nn.sql

li create_function_jittery_boston_expand.sql

i create_function_jittery_generate_scenarios_and_counts.sq1

i create_function_jittery_get_nn_to_sample_or_iteration.sql

$\backslash$ i create_function_jittery_median.sql

select jit_functions.generate_scenarios_and_counts('jittery', 26, 1320, 'k_25_qtrmi'); select jit_functions.generate_scenarios_and_counts('jittery', 51, 1320, 'k_50_qtrmi');

select jit_functions.generate_scenarios_and_counts('jittery', 101, 1320, 'k_100_qtrmi');

select jit_functions.generate_scenarios_and_counts('jittery', 251, 1320, 'k_250_qtrmi');

select jit_functions.generate_scenarios_and_counts('jittery', 501, 1320, 'k_500_qtrmi');

select jit_functions.generate_scenarios_and_counts('jittery', 26, 2640, 'k_25_halfmi');

select jit_functions.generate_scenarios_and_counts('jittery', 51, 2640, 'k_50_halfmi');

select jit_functions.generate_scenarios_and_counts('jittery', 101, 2640, 'k_100_halfmi'); 
select jit_functions.generate_scenarios_and_counts('jittery', 251, 2640, 'k_250_halfmi'); select jit_functions.generate_scenarios_and_counts('jittery', 501, 2640, 'k_500_halfmi'); select jit_functions.generate_scenarios_and_counts('jittery', 26, 5280, 'k_25_onemi'); select jit_functions.generate_scenarios_and_counts('jittery', 51, 5280, 'k_50_onemi'); select jit_functions.generate_scenarios_and_counts('jittery', 101, 5280, ' $\mathrm{k} \_100$ _onemi'); select jit_functions.generate_scenarios_and_counts('jittery', 251, 5280, 'k_250_onemi'); select jit_functions.generate_scenarios_and_counts('jittery', 501, 5280, 'k_500_onemi'); select jit_functions.generate_scenarios_and_counts('jittery', 26, 7920, 'k_25_threehalfmi'); select jit_functions.generate_scenarios_and_counts('jittery', 51, 7920, 'k_50_threehalfmi'); select jit_functions.generate_scenarios_and_counts('jittery', 101, 7920, 'k_100_threehalfmi'); select jit_functions.generate_scenarios_and_counts('jittery', 251, 7920, 'k_250_threehalfmi'); select jit_functions.generate_scenarios_and_counts('jittery', 501, 7920, 'k_500_threehalfmi'); select jit_functions.generate_scenarios_and_counts('jittery', 26, 10560, 'k_25_twomi'); select jit_functions.generate_scenarios_and_counts('jittery', 51, 10560, 'k_50_twomi'); select jit_functions.generate_scenarios_and_counts('jittery', 101, 10560, 'k_100_twomi'); select jit_functions.generate_scenarios_and_counts('jittery', 251, 10560, 'k_250_twomi'); select jit_functions.generate_scenarios_and_counts('jittery', 501, 10560, 'k_500_twomi');

create function jittery calculate inner_radius.sql CREATE OR REPLACE FUNCTION jit_functions.calculate_inner_radius ( tract area double precision, kstat double precision, household_count double precision )

RETURNS double precision

AS \$ $\$$

DECLARE

calcValue double precision;

BEGIN

-- calcValue $=\operatorname{sqrt}\left(\right.$ tract_area/pi ()$\left.* k s t a t / h o u s e h o l d \_c o u n t\right) * 1609.344 ;$-- this was using meters, will use feet instead.

calcValue $=\operatorname{sqrt}(($ tract_area/pi ()$) *($ kstat/household_count $)) * 5280$;

RETURN calcValue;

END;

\$ LANGUAGE plpgsql;

create function jittery make donuts.sql

CREATE OR REPLACE FUNCTION jit_functions.make_donuts ( input_schema text, ohas_points text, k_stat double precision, outer_radius double precision, output_schema text, debug boolean DEFAULT FALSE )

RETURNS Boolean 


\section{AS \$ \$}

DECLARE

to_execute text;

function_schema text:= 'jit_functions';

BEGIN

-- Create the table to hold inner-radius calculations

to_execute = 'DROP TABLE IF EXISTS ' || output_schema || '.inner_radius';

IF debug THEN

RAISE INFO '\%', to_execute;

END IF;

EXECUTE to execute;

to execute $=$ 'CREATE TABLE ' $\|$ output schema $\|$ '.inner buf radius AS

SELECT '|| function_schema $\|$ '.calculate_inner_radius(AREA_NOH2O, ' $\|$ k_stat $\|$ ', HU10_OCC) as inner_radius,

geo_tr.gid as geo_tr_id,

ohas.sampn as sampn

FROM ' || input_schema $\|$ '.ohas

JOIN ' $\|$ input_schema $\|$ '.geo_tr ON ST_Contains(geo_tr.geom, ohas.geom)';

IF debug THEN

RAISE INFO '\%', to_execute;

END IF;

EXECUTE to execute;

-- Creating a table of the inner \& outer ring geometry of each point.

to_execute $=$ 'DROP TABLE IF EXISTS ' || output_schema $\|$ '.rings';

IF debug THEN

END IF;

RAISE INFO '\%', to_execute;

EXECUTE to_execute;

to_execute $=$ 'CREATE TABLE' $\|$ output_schema $\|$ '.rings AS

SELECT ST_Buffer(ohas.geom, buff.inner_radius) as inner_ring,

ST_Buffer(ohas.geom, '|| outer_radius ||') as outer_ring,

ohas.sampn as sampn

FROM '|| input_schema || '.ohas

JOIN ' || output_schema || '.inner_buf_radius as buff on (ohas.sampn = buff.sampn)';

IF debug THEN

RAISE INFO '\%', to_execute;

END IF;

EXECUTE to_execute;

-- Clip the inner rings from the outer rings.

--Create a table of the "ring" geometry for each point.

to_execute = 'DROP TABLE IF EXISTS ' || output_schema || '.donuts';

IF debug THEN

RAISE INFO '\%', to_execute;

END IF;

EXECUTE to execute;

to_execute $=$ 'CREATE TABLE' $\|$ output_schema $\|$ '.donuts AS 
SELECT ST_Difference(rings.outer_ring, rings.inner_ring) as donut, rings.sampn

from '|| output_schema ||'.rings';

IF debug THEN

RAISE INFO '\%', to_execute;

END IF;

EXECUTE to_execute;

-- Clip the donuts to the tracts

to_execute $=$ 'DROP TABLE IF EXISTS ' || output_schema || '.donuts_clipped';

IF debug THEN

END IF;

RAISE INFO '\%', to_execute;

EXECUTE to_execute;

to_execute $=$ 'CREATE TABLE ' $\|$ output_schema $\|$ '.donuts_clipped AS

SELECT ST_Intersection(donuts.donut, geo_tr.geom) as geom, donuts.sampn, b.geo_tr_id

FROM '|| output_schema ||'.donuts

JOIN '|| output_schema ||'.inner_buf_radius b on (donuts.sampn = b.sampn)

JOIN '|| input_schema ||'.geo_tr on (b.

IF debug THEN

RAISE INFO '\%', to_execute;

END IF;

EXECUTE to_execute;

RETURN TRUE;

END;

\$ LANGUAGE plpgsql;

create function jittery random point nulls handled.sql

--ALMOST Courtesy of http://sorokine.blogspot.com/2011/05/postgis-function-for-randompoint.html

CREATE OR REPLACE FUNCTION jit_functions.random_point_nulls_handled ( geom Geometry

--OUT rpoint Geometry,

--OUT about_rpoint varchar(10)

)

RETURNS Geometry

AS \$\$

DECLARE

i INTEGER $:=0$;

$\mathrm{x} 0$ DOUBLE PRECISION;

$\mathrm{dx}$ DOUBLE PRECISION;

y0 DOUBLE PRECISION;

dy DOUBLE PRECISION;

xp DOUBLE PRECISION;

yp DOUBLE PRECISION;

rpoint Geometry := NULL;

geom_2d Geometry := NULL; 
maxiter int $:=5000$;

about_rpoint varchar(10):='UNKNOWN';

BEGIN

IF ST_IsEmpty(geom::geometry) $<>$ 't' THEN

geom_2d = ST_GeomFromEWKB(geom);

--RĀISE INFO' '2ND HERE \%', geom;

-- find envelope

$\mathrm{x} 0=\mathrm{ST} \_\mathrm{XMin}\left(\mathrm{geom} \_2 \mathrm{~d}\right)$;

$\mathrm{dx}=\left(\mathrm{ST} \_\mathrm{XMax}(\right.$ geom_2d $\left.)-\mathrm{x} 0\right)$;

$\mathrm{y} 0=\mathrm{ST} \_$YMin $($geom_2d $)$;

$\mathrm{dy}=\left(\mathrm{ST} \_\mathrm{YMax}\left(\mathrm{geom} \_2 \mathrm{~d}\right)-\mathrm{y} 0\right)$;

WHILE $\overline{\mathrm{i}}<\operatorname{maxiter} \mathrm{LOOP}$

$\mathrm{i}=\mathrm{i}+1$;

$\mathrm{xp}=\mathrm{x} 0+\mathrm{dx} * \operatorname{random}()$

yp $=$ y0 + dy * random( $)$;

rpoint $=$ ST_SetSRID( ST_MakePoint $(x p, y p)$, ST_SRID(geom_2d) );

EXIT WHEN ST Within( rpoint, geom 2d );

--RAISE INFO 'SECOND HERE with $\%, \%, \%, \%, \%, \%$ ', i, rpoint, x0, dx, xp, geom_2d; END LOOP;

ELSE

about_rpoint := 'EMPTY-POLY';

END IF;

IF $\mathrm{i}>=$ maxiter THEN

--RAISE INFO 'RandomPoint: number of interations exceeded ', maxiter;

-- force the point to be st pointonsurface so that it doesn't land outside the clipped donut.

rpoint $=$ st_pointonsurface $($ geom_2d $)$;

about_rpoint := '2MANY-ITER';

END IF;

RETURN rpoint;

END;

\$ \$ LANGUAGE plpgsql;

create function_jittery boston_nearest neighbor.sql

-- With some implementation tweaks, basic function courtesy of

http://www.bostongis.com/blog/index.php?/categories/7-nearest-neighbor

CREATE OR REPLACE FUNCTION jit_functions.pgis_fn_nn(

geoml geometry,

distguess double precision,

numnn integer,

maxslices integer,

lookupset varchar(150),

swhere varchar(5000),

sgid2field varchar(100),

sgeom2field varchar(100))

RETURNS SETOF pgis_nn AS

\$BODY\$ 


\section{DECLARE}

strsql text;

rec pgis_nn;

ncollected integer;

it integer;

--NOTE: it: the iteration we are currently at

--start at the bounding box of the object (expand 0) and move up until it has collected more objects than we need or it $=$ maxslices whichever event happens first

BEGIN

ncollected $:=0 ;$ it $:=0$;

WHILE ncollected $<$ numnn AND it $<=$ maxslices LOOP

strsql := 'SELECT currentit.' || sgid2field || ', st_distance(ref.geom, currentit.' || sgeom2field || ') as dist

FROM ' || lookupset || ' as currentit, (SELECT geometry("' || CAST(geom1 As text) || "') As geom) As ref

WHERE ' ||swhere || ' AND ST Expand(ref.geom, ' || CAST(distguess*it/maxslices As varchar(100)) \|| ') \&\& currentit.geom

AND jit_functions.expandoverlap_metric(ref.geom, currentit.geom, ' || CAST(distguess As varchar(200)) || ', ' || CAST(maxslices As varchar(200)) || ') = ' || CAST(it As varchar(100)) || '

ORDER BY st_distance(ref.geom, currentit.geom) LIMIT ' || CAST((numnn - ncollected) As varchar(200));

--RAISE NOTICE 'sql: \%', strsql;

--RAISE NOTICE 'it: \%', it;

--RAISE NOTICE 'ncollected: \%', ncollected;

--RAISE NOTICE 'ncollected: \%', ncollected;

-- I put this in

if strsql is null THEN

EXIT;

end if;

FOR rec in EXECUTE (strsql) LOOP

IF ncollected $<$ numnn THEN

ncollected $:=$ ncollected +1 ;

RETURN NEXT rec;

ELSE

EXIT;

END IF;

END LOOP;

it $:=$ it +1 ;

END LOOP;

END

\$BODY\$

LANGUAGE 'plpgsql' STABLE;

create function_jittery boston_call_nn.sql

-- With some implementation tweaks, basic function courtesy of http://www.bostongis.com/blog/index.php?/categories/7-nearest-neighbor 
CREATE OR REPLACE FUNCTION jit_functions.call_pgis_fn_nn( geom1 geometry, distguess double precision, numnn integer, maxslices integer, lookupset varchar(150), swhere varchar(5000), sgid2field varchar(100), sgeom2field varchar(100))

\section{RETURNS SETOF pgis_nn AS}

\$BODY\$

SELECT * FROM jit functions.pgis_fn_nn $(\$ 1, \$ 2, \$ 3, \$ 4, \$ 5, \$ 6, \$ 7, \$ 8)$; \$BODY\$

LANGUAGE 'sql' STABLE;

create function_jittery boston expand.sql

-- With some implementation tweaks, basic function courtesy of http://www.bostongis.com/blog/index.php?/categories/7-nearest-neighbor CREATE OR REPLACE FUNCTION jit_functions.expandoverlap_metric( a geometry, b geometry, maxe double precision, maxslice double precision)

RETURNS integer AS

\section{\$BODY\$}

BEGIN

FOR i IN 0..maxslice LOOP

IF st expand(a,maxe*i/maxslice) \&\& b THEN RETURN i;

END IF;

END LOOP;

RETURN 99999999;

END;

\$BODY\$

LANGUAGE 'plpgsql' IMMUTABLE;

create function_jittery get nn to sample_or_iteration.sql

CREATE OR REPLACE FUNCTION jit_functions.get_nn_to_sample_or_iteration ( input_schema text, output_schema text, input_points_table_name text, nn_points_table_name text, output_table_name text, is_iteration boolean, max_nn_dist_expected double precision DEFAULT 200000, maxslices integer DEFAULT 100, 
debug boolean DEFAULT FALSE

)

RETURNS Boolean

AS \$ $\$$

-- Inputs points should be OHAS or one of the iteration sets of points.

DECLARE

to_execute text;

function_schema text:= 'jit_functions';

nn_points_id_field text :='gid';

BEGIN

to_execute = 'DROP TABLE IF EXISTS ' ||output_schema || '.' || output_table_name;

IF debug THEN

RAISE INFO '\%', to_execute;

END IF;

EXECUTE to_execute;

IF is_iteration THEN

to_execute = 'CREATE TABLE ' ||output_schema $\|$ '.' $\|$ output_table_name $\|$ ' AS

SELECT pts.sampn as sampn,

pts.iter_num as iter_num,

(jit_functions.call_pgis_fn_nn(

pts.geom,' --geoml geometry,

|| max_nn_dist_expected ||', '||--distguess double precision,

'1, '|l --numnn integer,'

maxslices $\|$ ', ' ||$--$ maxslices integer,

"''|| input_schema || '.' || nn_points_table_name||"', '||--lookupset varchar(150),

"'true", '||--swhere varchar(5000),

"'gid", '||--sgid2field varchar(100),

"'geom"' ||-- sgeom2field varchar(100)

')).* FROM '|| output_schema ||'.'||input_points_table_name || 'pts';

ELSE

to_execute $=$ 'CREATE TABLE ' ||output_schema $\|$ '.' $\|$ output_table_name $\|$ ' AS

SELECT pts.sampn as sampn,

(jit_functions.call_pgis_fn_nn(

pts.geom,' --geom1 geometry,

|| max_nn_dist_expected ||','|| --distguess double precision,

'1, '|| --numnn integer,'

maxslices $\|$ ', ' $\mid-$--maxslices integer,

'"' ||input_schema || '.' || nn_points_table_name||"', '|| --lookupset varchar(150),

"'true", '|l--swhere varchar(5000),

"'gid", ' ||--sgid2field varchar(100),

"'geom"' || -- sgeom2field varchar(100)

')).* FROM '|| input_schema ||'.'||input_points_table_name || 'pts';

END IF;

IF debug THEN

END IF;

RAISE INFO '\%', to_execute; 
EXECUTE to_execute;

RETURN TRŪE;

END;

\$ \$ LANGUAGE plpgsql;

create function jittery generate scenarios and counts.sql

--Test case run:

-- select jit_functions.generate_scenarios_and_counts('jittery', 51, 1620, 'tmp_8');

CREATE OR REPLACE FUNCTION jit_functions.generate_scenarios_and_counts ( input_schema text, k_stat double precision, outer_radius double precision, output_schema text

)

RETURNS Boolean

AS \$\$

DECLARE

to_execute text;

function_schema text:= 'jit_functions';

data_schema text:= 'jittery';

num scenarios integer: $=50$;

BEGIN

-- 0. PREP SCHEMA

to_execute = 'DROP SCHEMA IF EXISTS ' ||output_schema || 'CASCADE';

RAISE INFO '\%', to_execute;

EXECUTE to execute;

to execute $=$ 'CREATE SCHEMA ' $\|$ output_schema ;

RAISE INFO '\%', to_execute;

EXECUTE to execute;

to_execute $=$ 'CREATE TABLE ' $\|$ output_schema $\|$ '.iteration_rows AS SELECT generate_series as iter_num FROM generate_series(1, '|| num_scenarios || ')' ;

RAISE INFO '\%', to_execute;

EXECUTE to execute;

-- 1. MAKE DONUTS

to execute $=$

'SELECT ' || function_schema || '.make_donuts ("' || input_schema || "', "ohas", '|| k_stat || ', ' || outer_radius ||', "' || output_schema ||'')';

RAISE INFO '\%', to_execute;

EXECUTE to execute;

-- 2. GENERATE SCENARIO POINTS

-- 2.a Create the points themselves

to - execute $=$

'CREATE TABLE ' || output_schema|| '.iteration_points AS SELECT ' || function_schema || '.random_point_nulls_handled(dc.geom) as geom, dc.sampn, dc.geo_tr_id, i.iter_num FROM '|| output_schema || '.donuts_clipped dc, ' || output_schema || '.iteration_rows i';

RAISE INFO '\%', to_execute; 
EXECUTE to_execute;

-- 2.b Append to points the basic info about it's error \& where it came from:

to - execute $=$

'CREATE TABLE ' || output_schema $\|$ '.iteration_points_estkanon as SELECT ip.geom, ip.sampn, ip.iter_num, st_x(ip.geom) as xcoord_1, st_y(ip.geom) as ycoord_1, blocks.tract10 as tract_1, blocks.blkgrp10 as group_1, pi()*((ST_Distance(ip.geom, op.geom) $\left./ 5280)^{\wedge} 2\right)^{*}($ tracts.HU10_OCC/tracts.area_noh2o) as est_kanon FROM ' \| output_schema || '.iteration_points ip JOIN ' || input_schema $\|$ '.geo_bg blocks on st_intersects (ip.geom, blocks.geom) JOIN ' $\|$ input_schema $\|$ '.geo_tr tracts on (blocks.tract10 $=$ tracts.tract10) JOIN ' || input_schema $\|$ '.ohas op on (ip.sampn = op.sampn)';

RAISE INFO '\%', to_execute;

EXECUTE to execute;

-- 3. GET STATS ONTO THE OHAS \& SCENARIO POINTS

-- 3.a.i Rail onto the OHAS points

to - execute $=$

'SELECT ' || function_schema || '.get_nn_to_sample_or_iteration("' || input_schema $\|$

"',"' || output_schema || "',"ohas", "be_r", "ohas_transit_rail_closest", false)';

RAISE INFO '\%', to_execute;

EXECUTE to_execute;

-- 3.a.ii Rail onto the iteration points

to execute $=$

'SELECT ' || function_schema || '.get_nn_to_sample_or_iteration("' || input_schema \|

"','" || output_schema || "',"iteration_points", "be_r", "ohas_iter_transit_rail_closest", true)';

RAISE INFO '\%', to_execute;

EXECUTE to execute;

-- 3.a.iii Calc the rail errors

to execute $=$

'CREATE TABLE ' $\|$ output_schema $\|$ '.ohas_jitter_rail_errors as ' $\|$

'SELECT ip.*, o.dist as be_0, j.dist as be_1,' $\|$

'(o.dist - j.dist) as abs_err, '

'((o.dist - j.dist) / o.dist) as rel_err, ' $\|$

'(abs(o.dist - j.dist) / o.dist) as absrel_err' $\mid$

'FROM ' || output_schema || '.iteration_points_estkanon ip JOIN '

$\|$ output_schema $\|$ '.ohas_transit_rail_closest o on $\left(\right.$ o.sampn $=$ ip.sampn) JOIN ${ }^{\prime}$

|| output_schema || '.ohas_iter_transit_rail_closest j ' $\|$

'ON (j.sampn = ip.sampn and j.iter_num = ip.iter_num)';

RAISE INFO '\%', to_execute;

EXECUTE to_execute;

-- 3.b.i Bus onto the ohas points

to execute $=$

'SELECT ' || function_schema || '.get_nn_to_sample_or_iteration("' || input_schema \| "','" || output_schema || "',"ohas", "be_b", "ohas_transit_bus_closest", false)';

RAISE INFO '\%', to_execute;

EXECUTE to_execute;

-- 3.b.ii Bus onto the iteration points

to $\_$execute $=$ 
'SELECT ' || function_schema || '.get_nn_to_sample_or_iteration("' || input_schema \|

"',"' || output_schema || "',"iteration_points", "be_b", "ohas_iter_transit_bus_closest", true)';

RAISE INFO '\%', to_execute;

EXECUTE to execute;

-- 3.b.iii Calc the bus errors

to execute $=$

'CREATE TABLE ' $\|$ output_schema $\|$ '.ohas_jitter_bus_errors as select ip.*, o.dist as be 0 , j.dist as be_1, (o.dist - j.dist) as abs_err, ((o.dist - j.dist) / o.dist) as rel_err, (abs(o.dist - j.dist) / o.dist) as absrel_err FROM ' $\|$ output_schema $\|$ '.iteration_points_estkanon ip JOIN ' $\|$ output_schema $\|$ '.ohas_transit_bus_closest o on $($ o.sampn $=$ ip.sampn) JOIN ' $\|$ output_schema $\|$ '.ohas_iter_transit_bus_closest $\mathbf{j}$ on $(\mathbf{j} . \mathrm{sampn}=\mathrm{ip} . \mathrm{sampn}$ and j.iter_num $=$ ip.iter_num)';

RAISE INFO '\%', to execute;

EXECUTE to_execute;

--3.c.i Block group variables on to ohas points

to execute $=$

'CREATE TABLE' || output_schema || '.ohas_bg_be_measures AS SELECT ohas.sampn as sampn, geo_bg.geoid as bg_geo_id, int_dens.inter_dens as be_0_int_dens, int_dens.pop_dens as be_0_pop_dens, int_dens.emp_dens as be_0_emp_dens FROM ' $\mid$ '

input_schema $\|$ '.ohas JOIN ' $\|$ input_schema $\|$ '.geo_bg on ST_Within(ohas.geom, geo_bg.geom) JOIN ' $\|$

input schema $\|$ '.int dens on (geo_bg.geoid = int_dens.geoid)';

RAISE INFO '\%', to execute;

EXECUTE to_execute;

$--3 . c . i i$ Block group variables onto the iteration points

to - execute $=$

'CREATE TABLE ' $\|$ output_schema || '.ohas_iter_bg_be_measures AS SELECT ip.sampn as sampn, ip.iter_num as iter_num, geo_bg.geoid as bg_geo_id, int_dens.inter_dens as be_1_int_dens, int_dens.pop_dens as be_1_pop_dens, int_dens.emp_dens as be_1_emp_dens FROM '\|

output_schema || '.iteration_points ip JOIN ' $\|$ input_schema $\|$ '.geo_bg on ST_Within(ip.geom, geo_bg.geom) JOIN ' $\|$ input_schema || 'int_dens on (geo_bg.geoid = int_dens.geoid)';

RAISE INFO '\%', to_execute;

EXECUTE to_execute;

$--3 . c . i i i . a$ Block group variables errors - intersection density

to execute $=$

'CREATE TABLE ' $\|$ output_schema $\|$ '.ohas_jitter_int_errors as select ip.*, o.be_0_int_dens as be_0,j.be_1_int_dens as be_1, (j.be_1_int_dens - o.be_0_int_dens) as abs_err, ((j.be_1_int_dens - o.be_0_int_dens) / o.be_0_int_dens $)$ as rel_err, abs $\left(\left(\left(\bar{j} \cdot b e \_1\right.\right.\right.$ int dens o.be_0_int_dens) / o.be_0_int_dens)) as absrel_err FROM ' \| output_schema \| '.iteration_points_estkanon ip JOIN ' $\|$ output_schema $\|$ '.ohas_bg_be_measures o on (o.sampn $=$ ip.sampn) JOIN ' ${ }^{\top} \|$ output_schema $\|$ '.ohas_iter_bg_be_measures $\mathrm{j}$ on $(\mathrm{j} . \mathrm{sampn}=\mathrm{ip}$.sampn and j.iter_num $=$ ip.iter_num)';

RAISE INFO '\%', to_execute;

EXECUTE to_execute;

--3.c.iii.b Block group variables errors - pop density 
to_execute $=$

'CREATE TABLE ' $\|$ output_schema $\|$ '.ohas_jitter_pop_errors as select ip.*, o.be_0_pop_dens as be_0, j.be_1_pop_dens as be_1, (j.be_1_pop_dens - o.be_0_pop_dens) as abs_err, ((j.be_1_pop_dens - o.be_0_pop_dens) / o.be_0_pop_dens) as rel_err, abs(((j.be_1_pop_dens - o.be_0_pop_dens) / o.be_0_pop_dens)) as absrel_err FROM ${ }^{-}$" $\|$ output_schema $\|{ }^{\top}$.iteration_points_estkanon ip JOIN ${ }^{-} \|$output_schema $\|^{\prime}$ 'ohas_bg_be_measures o on $($ o.sampn $=$ ip.sampn) JOIN ' || output_schema $\|$ '.ohas_iter_bg_be_measures j on (j.sampn $=$ ip.sampn and j.iter_num $=$ ip.iter_num)';

RAISE INFO '\%', to_execute;

EXECUTE to execute;

--3.c.iii.c Block group variables errors - emp density

to execute $=$

'CREATE TABLE'

|| output_schema || 'ohas_jitter_emp_errors as select ip.*,o.be_0_emp_dens as be_0, j.be_1_emp_dens as be_1, (j.be_1_emp_dens - o.be_0_emp_dens) as abs_err, ((j.be_1_emp_dens - o.be_0_emp_dens $) /$ o.be_0_emp_dens $)$ as rel_err, abs $\left(\left(\left(\mathrm{j} . \bar{b} e \_1\right.\right.\right.$ emp_dens - o.be_0_emp_dens $)$

/ o.be 0 emp dens)) as absrel err FROM '

|| output_schema || '.iteration_points_estkanon ip JOIN '

|| output_schema || '.ohas_bg_be_measures o on (o.sampn=ip.sampn) JOIN '

\| output_schema $\|$ '.ohas_iter_bg_be_measures j on $(\mathrm{j} . \mathrm{sampn}=\mathrm{ip} . \mathrm{sampn}$ and j.iter_num $=$ ip.iter_num)';

RAISE INFO '\%', to_execute;

EXECUTE to_execute;

-- 4. SUMMARY STATS

-- 4.a basic info about the ohas points

to_execute $=$

'CREATE TABLE '

|| output_schema || '.ohas_info as ' $\|$

'SELECT o.sampn, o.geom, st_x(o.geom) as xcoord_0, st_y(o.geom) as ycoord_0, ' $\|$

'blocks.tract10 as tract_0, blocks.blkgrp10 as group_0 FROM '

|| input_schema || '.ohas o JOIN '

|| input_schema || '.geo_bg blocks on st_intersects (o.geom, blocks.geom) JOIN '

$\|$ input_schema $\|$ '.geo_tr tracts on (blocks.tract10 = tracts.tract10)';

RAISE INFO ' $\%$ ', to_execute;

EXECUTE to execute;

--4.a.i RMSE tables - emp

to_execute $=$

'CREATE TABLE '|| output_schema || '.rmse_emp as select '||

'min(oi.sampn) as sampn, ' $\|$

'min(oi.tract_0) as tract_0, '

'min(oi.group_0) as group_0,' ||

'min(oi.xcoord_0) as xcoord_0, '\|

'min(oi.ycoord_0) as ycoord_0, 'I

'min(est_kanon) as min_kanon, '

'jit_functions.median(est_kanon::numeric) as med_kanon, '|l

'max(est_kanon) as max_kanon, 'I 
'avg(est_kanon) as mean_kanon, '\|

'count(je.sampn) as num_sims, '||

'min(je.be_0) as be_0, 'II

'avg(je.be_1) as mean_be_1, '\|

'min(je.be_1) as min_be_1, '\|

'jit_functions.median(je.be_1) as med_be_1, '\|

'max(je.be_1) as max_be_1, '\|

'sum(abs(abs_err))/count(je.sampn) as mae, ' $\|$

'sqrt((sum(abs_err^ 2$)) /$ count(je.sampn)) as rmse, ' $\|$

'sqrt((sum(abs_err ^ 2$)) / \operatorname{count}($ je.sampn) $) / \operatorname{avg}($ be_1) as nrmse, ' $\|$

'sqrt((sum(abs_err ^ 2$)) / \operatorname{count}($ je.sampn) $) / \operatorname{avg}\left(b_{-}{ }_{-} 1\right) * 100$ as prmse from ' $\|$

output_schema || '.ohas_info oi ' $\|$

'LEFT_JOIN ' || output_schema || '.ohas_jitter_emp_errors je ' ||

'on (oi.sampn = je.sampn) group by oi.sampn';

RAISE INFO '\%', to_execute;

EXECUTE to execute;

--4.a.ii RMSE tables - int

to execute $=$

'CREATE TABLE '|| output_schema $\|$ '.rmse_int as select '|| 'min(oi.sampn) as sampn, ' $\|$

'min(oi.tract_0) as tract_ 0, ' $\|$

'min(oi.group_0) as group_0, ' $\|$

'min(oi.xcoord_0) as xcoord_0, '||

'min(oi.ycoord_0) as ycoord_0, '\|

'min(est_kanon) as min_kanon, '\|

'jit_functions.median(est_kanon::numeric) as med_kanon, '||

'max(est kanon) as max kanon, '||

'avg(est_kanon) as mean_kanon, '||

'count(je.sampn) as num_sims, '|l

'min(je.be_0) as be_0, 'll

'avg(je.be_1) as mean_be_1, '\|

'min(je.be_1) as min_be_1, 'II

'jit_functions.median(je.be_1) as med_be_1, 'II

'max(je.be_1) as max_be_1, '\|

'sum(abs(abs_err))/count(je.sampn) as mae, ' $\|$

'sqrt((sum(abs_err $\left.\left.{ }^{\wedge} 2\right)\right) / \operatorname{count}($ je.sampn $\left.)\right)$ as rmse, ' $\|$

'sqrt((sum(abs_err $\left.\left.{ }^{\wedge} 2\right)\right) / \operatorname{count}($ je.sampn $\left.)\right) / \operatorname{avg}($ be_1) as nrmse, ' $\|$

'sqrt((sum(abs_err ^ 2$)) / \operatorname{count}($ je.sampn) $) / \operatorname{avg}\left(b_{-}{ }_{-} 1\right) * 100$ as prmse from ' $\|$

output_schema $\|$ '.ohas_info oi ' $\|$

'LEFT JOIN ' || output_schema || '.ohas_jitter_int_errors je ' ||

'on (oi.sampn = je.sampn) group by oi.sampn';

RAISE INFO '\%', to_execute;

EXECUTE to_execute;

--4.a.iii RMSE tables - pop

to execute $=$

'CREATE TABLE '|| output_schema || '.rmse_pop as select '|| 


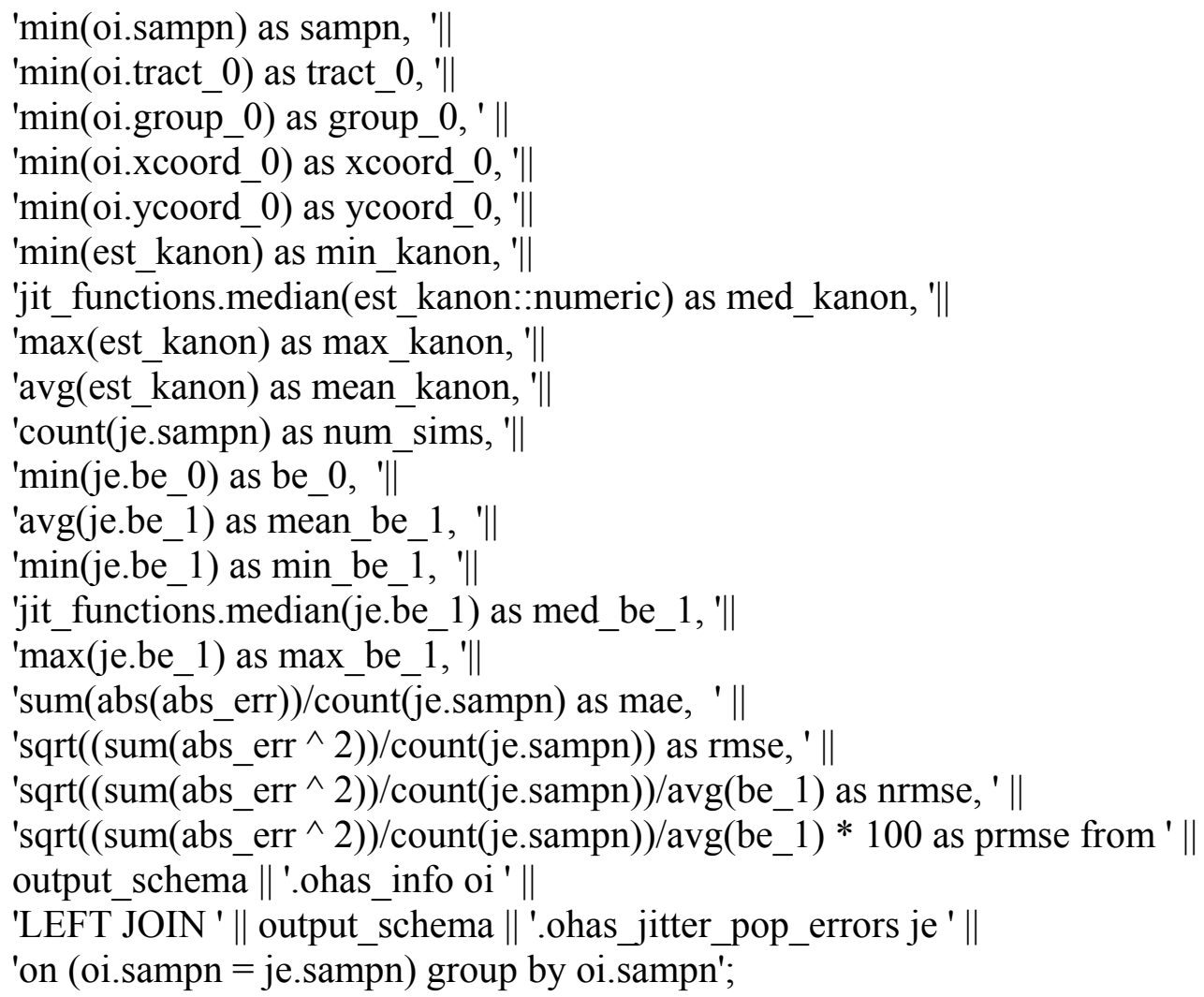


output_schema || '.ohas_info oi ' ||

'LEFT JOIN ' || output_schema || '.ohas_jitter_rail_errors je ' ||

'on (oi.sampn = je.sampn) group by oi.sampn';

RAISE INFO '\%', to_execute;

EXECUTE to_execute;

--4.a.v RMSE tables - bus

to - execute $=$

'CREATE TABLE '|| output_schema || '.rmse_bus as select '||

'min(oi.sampn) as sampn, 'I|

'min(oi.tract_0) as tract_ 0, ' $\|$

'min(oi.group_0) as group_0, ' $\|$

'min(oi.xcoord_0) as xcoord_0, '||

'min(oi.ycoord_0) as ycoord_0, '||

'min(est_kanon) as min_kanon, '\|

'jit_functions.median(est_kanon::numeric) as med_kanon, '||

'max(est_kanon) as max_kanon, '||

'avg(est kanon) as mean_kanon, '||

'count(je.sampn) as num_sims, 'I|

'min(je.be_0) as be_0, 'll

'avg(je.be_1) as mean_be_1, 'I|

'min(je.be_1) as min_be_ 1 , ' $\|$

'jit_functions.median(je.be_1) as med_be_1, '\|

'max(je.be_1) as max_be_1, '|l

'sum(abs(abs_err))/count(je.sampn) as mae, ' $\|$

'sqrt((sum(abs_err ^ 2$)) /$ count(je.sampn)) as rmse, ' $\|$

'sqrt((sum(abs_err ^ 2$)) / \operatorname{count}($ je.sampn) $) / \operatorname{avg}($ be_1) as nrmse, ' $\|$

'sqrt((sum(abs_err^^2))/count(je.sampn))/avg(be_1) * 100 as prmse from ' $\|$

output_schema $\|$ '.ohas_info oi ' ||

'LEFT JOIN ' || output_schema \|' '.ohas_jitter_bus_errors je ' $\|$

'on (oi.sampn = je.sampn) group by oi.sampn';

RETURN TRUE;

END;

\$ LANGUAGE plpgsql;

create function jittery median.sql

-- From http://wiki.postgresql.org/wiki/Aggregate_Median

CREATE OR REPLACE FUNCTION jit_functions._final_median(numeric[])

RETURNS numeric AS

$\$ \$$

SELECT AVG(val)

FROM (

SELECT val

FROM unnest $(\$ 1)$ val

ORDER BY 1

LIMIT 2 - MOD(array_upper( $\$ 1,1), 2)$

OFFSET CEIL(array_upper( $(\$ 1,1) / 2.0)$ - 1 


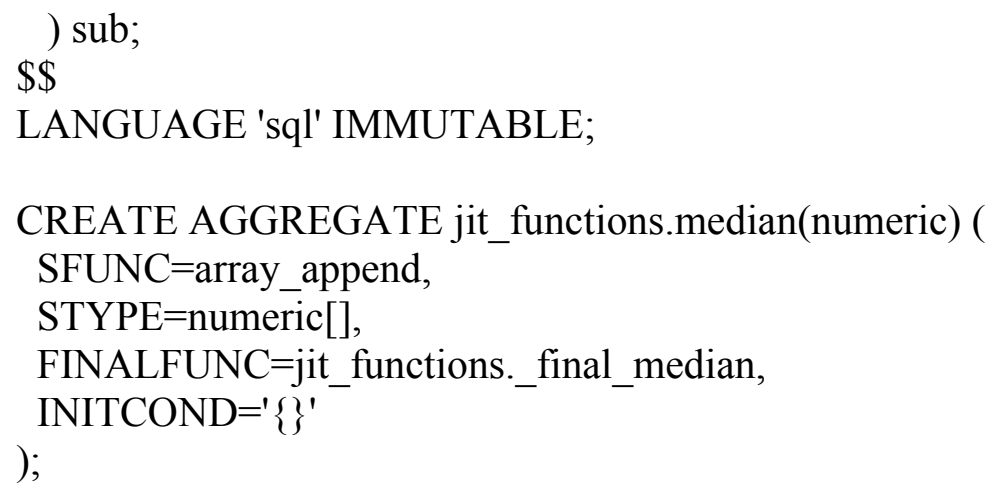


!! pgsq12shp -f \{MYSCHEMA\}/donuts_clipped -h localhost omc "SELECT ST_Multi(geom) as geom, sampn, geo_tr_id FROM \{MYSCHEMA\}.donuts_clipped WHERE not ST_IsEmpty(geom)"

! zip -qrv \{MYSCHEMA .zip \{MYSCHEMA $\}$ /*

! rm -rf \{MYSCHEMA\} 



\section{GOTREC \\ AND EDUCATION CONSORTIUM}

P.O. Box 751

Portland, OR 97207

OTREC is dedicated to stimulating and conducting collaborative multi-disciplinary research on multi-modal surface transportation issues, educating a diverse array of current practitioners and future leaders in the transportation field, and encouraging implementation of relevant research results. 